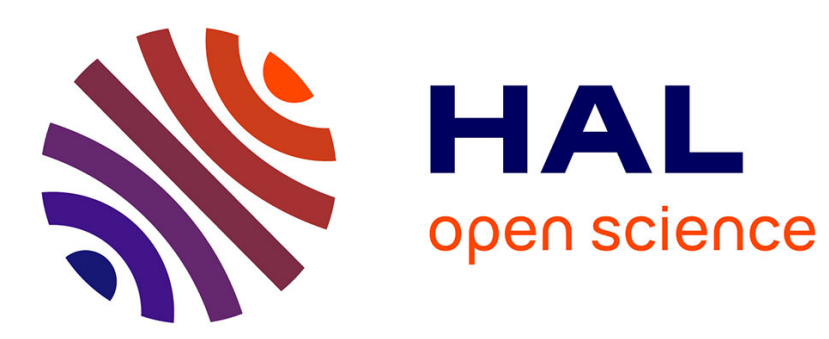

\title{
Homogenization of a Large Eddy Simulation Model for Turbulent Fluid Motion near a Rough Wall
}

\author{
Matthieu Bonnivard, Francisco J Suarez-Grau
}

\section{To cite this version:}

Matthieu Bonnivard, Francisco J Suarez-Grau. Homogenization of a Large Eddy Simulation Model for Turbulent Fluid Motion near a Rough Wall. 2018. hal-01696924

\section{HAL Id: hal-01696924 \\ https://hal.science/hal-01696924}

Preprint submitted on 30 Jan 2018

HAL is a multi-disciplinary open access archive for the deposit and dissemination of scientific research documents, whether they are published or not. The documents may come from teaching and research institutions in France or abroad, or from public or private research centers.
L'archive ouverte pluridisciplinaire HAL, est destinée au dépôt et à la diffusion de documents scientifiques de niveau recherche, publiés ou non, émanant des établissements d'enseignement et de recherche français ou étrangers, des laboratoires publics ou privés. 


\title{
Homogenization of a Large Eddy Simulation Model for Turbulent Fluid Motion near a Rough Wall
}

\author{
Matthieu Bonnivard* $\quad$ Francisco J. Suárez-Grau ${ }^{\dagger}$
}

September 6, 2017

\begin{abstract}
In this paper, we are interested in the numerical simulation of a turbulent viscous flow in the vicinity of a periodic rough wall. To this aim, we consider a Large Eddy Simulation (LES) model, and perform its asymptotic analysis, letting the period $\varepsilon$ of the roughness tend to zero. Relying on an homogenization method closely related to the two-scale convergence method $[2,38]$, we exhibit a critical scaling associated with parameter $\lambda:=\lim _{\varepsilon \rightarrow 0} a_{\varepsilon} / \varepsilon^{5 / 3}$, where $a_{\varepsilon}$ stands for the amplitude of the roughness. In the critical regime $0<\lambda<\infty$, the roughness effect is described by an additional, nonlinear friction term, that depends locally on the solution to a nonlinear auxiliary problem of boundary layer type. In the particular case of riblets, which is of major practical interest, we show that the directional invariance of the geometry results in a simplified expression of this extra term, and we propose a numerical method to simulate the homogenized LES model, based on that observation. Finally, we study the influence of parameter $\lambda$ on the flow approximated by the homogenized model, using different commonly used riblet shapes. Our simulations demonstrate that the extra friction term can be handled numerically, and may have an important influence on the results of LES in presence of roughness.
\end{abstract}

AMS Classification: 35B27, 76F65.

Keywords: turbulence model; large-eddy simulation; roughness effect; homogenization.

\section{Introduction}

The study of roughness effects in fluid-structure interaction has seen considerable development in the last decades. Its main objective is to explain how the fine structure of solid walls may influence viscous flows in their vicinity, and how the effects of surface irregularities should be taken into account in order to enhance the precision of the existing models. One of the first mathematical results in this direction concerns the justification of the no-slip boundary condition, which is commonly used in continuum mechanics models and prescribes a null velocity for the fluid particles along the solid boundaries. In their pioneering paper [16], Casado-Díaz et al. have explained that boundary condition using an asymptotic analysis based on the presence of small-scale irregularities on the wall, and on a minimal boundary condition - the non

\footnotetext{
${ }^{*}$ Univ. Paris Diderot, Sorbonne Paris Cité, Laboratoire Jacques-Louis Lions, UMR 7598, UPMC, CNRS, F-75205 Paris, France

${ }^{\dagger}$ Dpto. de Ecuaciones Diferenciales y Análisis Numérico, Fac. de Matemáticas, Univ. de Sevilla
} 
penetration condition - expressing the impossibility for the fluid to get through the solid. In a simplified manner, that phenomenon could be interpreted as follows: the rough geometrical structure of the wall causes the normal $n$ to the boundary to oscillate with high spatial frequency, turning non penetration condition $u \cdot n=0$ into no-slip condition $u=0$, where $u$ stands for the fluid velocity.

The study of such rugosity effects is of particular importance in the field of microfluidics. Indeed, when fluid-structure interaction occurs at micro or nanoscale, surface effects become prominent due to miniaturization, and may have a strong impact on the behaviour of the fluid. At those scales, positive slip lengths have been measured experimentally, which justify the introduction of Navier boundary conditions in the Stokes models used to describe that type of configuration. We refer to [32] for a review of such experimental results. In the framework of fluid-solid contact with slip, numerous mathematical results have been obtained, that draw a rather complete picture of the effects that rough walls may produce on viscous flows at low Reynolds number. The stronger effect consists in forcing the fluid velocity to vanish, in the limit where the characteristic dimensions of the roughness go to zero, as was previously described. The rugosity effect can also be directional, when the surface texture forces the fluid to flow in a specific direction, tangential to the wall. Last, rough walls may produce an extra friction coefficient (or in other words, reduce the slip length), potentially anisotropic. We refer to [14] for a general mathematical description of those effects, that can also be combined.

By specifying particular types of rough patches, it is possible to quantify precisely such effects, especially by computing friction coefficients. One typical example is the case of periodic distributions of roughness, that can be treated by computating a friction matrix, whose coefficients are determined by solving boundary layer problems $[17,28]$. Among such periodical roughness profiles, the riblets, which are assemblies of the same elongated structure, are of considerable interest. They are widely used in engineering, extensively studied in experiments and mechanical models (see $[7,8,25,30,33]$ ) and have been the subject to many mathematical studies (see, for instance, $[3,13,29]$ ). However, in many applications such as aeronautics [45] or shark-skin drag reduction [20], rugosity effects (and especially, riblet effects) occur in turbulent regime. At present, a rigourous mathematical analysis of fluid-structure interaction at high Reynolds number remains out of reach, due to the well-known, tremendous difficulties posed by the three-dimensional Navier-Stokes equations in that context. From a numerical point of view, the direct numerical simulation of those equations, at high Reynolds number, can be very expensive, since it requires solving a very wide range of time and length scales - whose presence is characteristic of turbulence. As a result, lots of tools and models have been developed to describe and simulate turbulent flows, that are commonly used in mechanics [39].

In this paper, we focus on the class of Large Eddy Simulation (LES) models, that were introduced by Smagorinsky in 1963 [42] and are widely used in computational fluid dynamics. The principle of LES is to apply a spatial convolution operator to the Navier-Stokes equations, in order to decompose the velocity, pressure and body forces into two parts: an averaged part describing the global, large scale behaviour of the fluid, and a remainder corresponding to the small scales. The goal of LES models is to compute only the large scale structure of the flow. However, due to the presence of the nonlinear convective term in the moment equation, there is an interaction between small and large scales that one needs to model in order to close the average Navier Stokes system of equations. This well-known closure problem has led to a large variety of LES models, whose derivation can be motivated by physical or by mathematical considerations. The mathematical approach is based on the use of the Fourier transform, as will be detailed in Section 2. We refer to [46] and the references therein for a description of the main LES models based on this approach.

In this work, we address the problem of simulating numerically the turbulent flow of a viscous fluid, in the vicinity of a rough wall, using one of the simplest and most popular LES model: the Smagorinsky 
model. As was proved by Coletti in [19], this model enjoys nice mathematical properties, in particular, global existence and uniqueness of a weak solution. In order to develop an asymptotic analysis of the model, with respect to the characteristic length of the rough pattern, we rely on a remark by Galdi and Layton [24], which is also developed in [46] (Chap. 5). Starting from the observation that the noslip boundary condition, which is the commonly accepted boundary condition for viscous flows at high Reynolds number, cannot be preserved by spatially filtering the velocity using convolution, the previous authors have proposed to equip LES models with Navier boundary conditions. As stated in the beginning of this Introduction, such boundary conditions are well adapted to the study of rugosity effects. As a matter of fact, we are able to generalize the multi-scale analysis developed in [17], to a time-dependant system of equations, involving a nonlinear stress tensor, in the case of a periodic rough wall. We identify a new critical scaling between the amplitude and the period of the oscillations, and obtain an effective system set in the homogenized domain, that expresses the effect of the rough patches on the LES model, by adding a nonlinear interaction term to the initial boundary condition of Navier type. In this effective system, the homogenized boundary condition is determined locally by solving a boundary layer problem that involves only the nonlinear part of the stress tensor - that is, the part modelling the large scales/small scales interactions.

Since our work is motivated by the numerical simulation of turbulent flow near a rough wall, we conclude by addressing a particular geometric configuration, which is widely studied in the experimental fluid mechanics literature, and turns out to be well-adapted for simulating the limit system: the case of riblets. Indeed, using their directional invariance, we are able to obtain a simplified expression of the extra friction term, that requires the solving of only two different auxiliary problems. Then, we propose a numerical method to solve the limit LES model, based on a fixed point algorithm for treating the nonlinear part of the stress tensor, and the nonlinear friction term accounting for the rugosity effect. We implement that method to simulate the steady-state of the LES model, using three different riblet shapes that are commonly used in the literature, and discuss the influence of parameter $\lambda$ for these geometries. Our simulations demonstrate the extra friction term emerging from the asymptotic analysis of the model, can be taken into account in the applications, and have a non neglictable impact on the physical properties of the flow approximated by Large Eddy Simulation.

The paper is organized as follows. The Smagorinsky model is presented in Sec. 2. The main results are stated in Sec. 3, and proved in Sec. 4. Finally, Sec. 5 is dedicated to numerics.

\section{The Smagorinsky model for turbulent flow}

We consider the flow of a viscous and homogeneous fluid, driven by an external force, in a threedimensional domain delimited by two fixed horizontal plates. We assume that the upper plate is perfectly smooth, but that the lower one presents a periodic rough surface. We work in nondimensionalized coordinates and denote by Re the Reynolds number associated with the flow.

Let us start with the geometric notation that will be used throughout the paper.

\subsection{Geometric notation}

We denote by $\left(e_{1}, e_{2}, e_{3}\right)$ the canonical basis of $\mathbb{R}^{3}$ and by $x=\left(x_{1}, x_{2}, x_{3}\right)$ the spatial coordinates. The top smooth boundary occupies the plane $x_{3}=1$, with normal $e_{3}$. For the sake of simplicity, we assume that the flow is periodic with respect to the horizontal coordinates $\left(x_{1}, x_{2}\right)$, which motivates the introduction of the tori $\mathcal{T}^{2}:=\mathbb{R}^{2} / \mathbb{Z}^{2}$ and $\mathcal{T}^{1}:=\mathbb{R} / \mathbb{Z}$.

To describe the geometry of the lower (rough) plate, we fix a positive function $\Psi \in W^{2, \infty}\left(\mathcal{T}^{2}\right)$ and define

$$
\Psi_{\varepsilon}\left(x_{1}, x_{2}\right)=a_{\varepsilon} \Psi\left(\frac{x_{1}}{\varepsilon}, \frac{x_{2}}{\varepsilon}\right), \quad \text { a.e. }\left(x_{1}, x_{2}\right) \in \mathcal{T}^{2},
$$


where $\varepsilon>0$ is a small parameter and $a_{\varepsilon}$ satisfies

$$
\lim _{\varepsilon \rightarrow 0} a_{\varepsilon}=0, \quad \lim _{\varepsilon \rightarrow 0} \frac{a_{\varepsilon}}{\varepsilon}=0 .
$$

The rough boundary $\Gamma_{\varepsilon}$ is then defined by

$$
\Gamma_{\varepsilon}:=\left\{\left(x_{1}, x_{2}, x_{3}\right) \in \mathcal{T}^{2} \times \mathbb{R}: x_{3}=-\Psi_{\varepsilon}\left(x_{1}, x_{2}\right)\right\} .
$$

By the periodicity of $\Psi, \Psi_{\varepsilon}$ is periodic of period $\varepsilon$ in the $x_{1}$ and $x_{2}$ directions, and by construction, the maximum value of $\Psi_{\varepsilon}$ is proportional to $a_{\varepsilon}$. Thus, hypotheses (2.1) mean that the amplitude of the roughness is negligible with respect to the period, as the period tends to zero.

Let $\nu$ the be the outside unitary normal vector to $\Omega_{\varepsilon}$ on $\Gamma_{\varepsilon}$. The orthogonal projection on the tangent space of $\partial \Omega_{\varepsilon}$ will be denoted by $T$, i.e.

$$
T \xi=\xi-(\xi \cdot \nu) \nu, \quad \forall \xi \in \mathbb{R}^{3}, \text { a.e. on } \Gamma_{\varepsilon} .
$$

We denote by $\Gamma^{0}$ the upper (smooth) boundary, defined by

$$
\Gamma^{0}:=\mathcal{T}^{2} \times\{1\}
$$

and introduce the fluid domain $\Omega_{\varepsilon}$ defined by

$$
\Omega_{\varepsilon}:=\left\{\left(x_{1}, x_{2}, x_{3}\right) \in \mathcal{T}^{2} \times \mathbb{R}:-\Psi_{\varepsilon}\left(x_{1}, x_{2}\right)<x_{3}<1\right\} .
$$

Since the sequence $\left(\Psi_{\varepsilon}\right)_{\varepsilon>0}$ converges uniformly to zero, the sequence $\left(\Gamma_{\varepsilon}\right)_{\varepsilon>0}$ converges in the sense of Hausdorff to the flat wall $\Gamma$ defined by

$$
\Gamma=\mathcal{T}^{2} \times\{0\}
$$

Accordingly, we introduce the limit domain

$$
\Omega=\mathcal{T}^{2} \times(0,1)
$$

and define $\Lambda$ by

$$
\Lambda=\mathcal{T}^{2} \times(-1,1),
$$

so that $\Lambda$ contains all the rough domains $\Omega_{\varepsilon}$.

\subsection{Derivation of the LES model}

Let $u_{\varepsilon}$ be the nondimensionalized velocity of the fluid and $p_{\varepsilon}$ the nondimensionalized pressure. We assume that the external force is given by the restriction to $\Omega_{\varepsilon}$ of a vector field $f \in L^{2}\left(\Lambda, \mathbb{R}^{3}\right)$. The fluid motion is described by the Navier-Stokes equations

$$
\begin{aligned}
\partial_{t} u_{\varepsilon}+\operatorname{div}\left(u_{\varepsilon} \otimes u_{\varepsilon}\right)-\frac{1}{R e} \Delta u_{\varepsilon}+\nabla p_{\varepsilon}=f & \text { in }] 0, T\left[\times \Omega_{\varepsilon},\right. \\
\operatorname{div} u_{\varepsilon}=0 & \text { in }] 0, T\left[\times \Omega_{\varepsilon},\right.
\end{aligned}
$$

completed with the initial condition

$$
u_{\varepsilon}(x, 0)=u_{\varepsilon}^{0}(x), \quad x \in \Omega_{\varepsilon}
$$


and no-slip boundary conditions on the plates:

$$
\begin{array}{ll}
u_{\varepsilon}=0 & \text { on } \Gamma^{0}, \\
u_{\varepsilon}=0 & \text { on } \Gamma_{\varepsilon} .
\end{array}
$$

As it is well known, in the three-dimensional case, existence and uniqueness of the solution of (2.5)(2.7) has only been proved for small Reynolds number [31]. For large values of Re, analytical solutions of Navier-Stokes equations become unstable; this is called turbulence. Our aim is to investigate numerically the influence of the rough boundary on turbulent states of the system. Although several numerical procedures have been developed to solve equations (2.5)-(2.6), they suffer the same instability issues as the analytical solutions, when the Reynolds number becomes large. To enhance the stability of the numerical solutions, one needs to consider a very fine mesh of the space domain, which increases the storage and computational costs dramatically, especially when the boundary of the domain presents fast oscillations.

As an alternative to direct numerical simulation (DNS), several models of turbulent flows have been developed. We refer to [37] for an introduction to the main models of turbulence. In order to simulate flows at high Reynolds number, we will apply the space filtered approach to large eddy simulation (LES), introduced in 1970 by Deardorff [21]. Let us recall the main steps of the derivation of this model; for more details on the derivation of classical LES models, we refer to $[9,19,40]$.

The spatial averaging operator is defined as a convolution with the Gaussian filter

$$
g_{\delta}(x):=\left(\frac{\gamma}{\pi}\right)^{3 / 2} \frac{1}{\delta^{3}} \exp \left[-\gamma|x|^{2} / \delta^{2}\right]
$$

where $\delta>0$ represents an averaging radius and $\gamma>0$ is a constant. All dependent variables being extended by zero to $\mathcal{T}^{2} \times \mathbb{R}$, the average of any variable $r(t, x)$ is defined by

$$
\bar{r}(t, x)=\left(g_{\delta} * r\right)(t, x)=\int_{\mathbb{R}^{3}} g_{\delta}(x-y) r(t, y) d y .
$$

Applying this averaging operator to system (2.5)-(2.7) and commuting spatial partial derivatives with convolutions, we formally obtain the system

$$
\begin{aligned}
\partial_{t} \bar{u}_{\varepsilon}+\operatorname{div}\left(\overline{u_{\varepsilon} \otimes u_{\varepsilon}}\right)-\frac{1}{R e} \Delta \bar{u}_{\varepsilon}+\nabla \bar{p}_{\varepsilon} & =\bar{f} \quad \text { in }] 0, T\left[\times \Omega_{\varepsilon},\right. \\
\operatorname{div} \bar{u}_{\varepsilon} & =0 \quad \text { in }] 0, T\left[\times \Omega_{\varepsilon},\right. \\
\bar{u}_{\varepsilon}(x, 0) & =\overline{u_{\varepsilon}^{0}}(x), \quad x \in \Omega_{\varepsilon} .
\end{aligned}
$$

However, the nonlinear term $\operatorname{div}\left(\overline{u_{\varepsilon} \otimes u_{\varepsilon}}\right)$ cannot be expressed as a function of the new unknown $\bar{u}_{\varepsilon}$. This notorious closure problem prevents us from solving the averaged problem (2.10)-(2.12) directly. To overcome this difficulty, it is required to derive an approximate expression of $\overline{u_{\varepsilon} \otimes u_{\varepsilon}}$, involving only the average velocity field $\bar{u}_{\varepsilon}$. Introducing the small-scale velocity $u_{\varepsilon}^{\prime}$ defined by

$$
u_{\varepsilon}=\bar{u}_{\varepsilon}+u_{\varepsilon}^{\prime}
$$

one starts with the following development:

$$
\overline{u_{\varepsilon} \otimes u_{\varepsilon}}=\overline{\bar{u}_{\varepsilon} \otimes \bar{u}_{\varepsilon}}+\overline{\bar{u}_{\varepsilon} \otimes u_{\varepsilon}^{\prime}}+\overline{u_{\varepsilon}^{\prime} \otimes \bar{u}_{\varepsilon}}+\overline{u_{\varepsilon}^{\prime} \otimes u_{\varepsilon}^{\prime}} .
$$

The principle of the approximation is to apply the Fourier transform to every term on the right hand side of (2.13), to exploit its commutation properties with respect to convolution, and to use a formal 
asymptotic development of the the result with respect to the small-scale $\delta$, as $\delta$ tends to zero, before returning to space variables by applying the inverse Fourier transform. Denoting by $\mathcal{F}$ the Fourier transform on $\mathcal{T}^{2} \times \mathbb{R}$ and by $\xi$ the dual variable, the Fourier transform of the Gaussian filter is defined by

$$
\widehat{g_{\delta}}(\xi):=\left[\mathcal{F}\left(g_{\delta}\right)\right](\xi)=\exp \left[-\frac{\delta^{2}}{4 \gamma}|\xi|^{2}\right],
$$

and using the properties of the convolution, there holds

$$
\mathcal{F}\left(\overline{\bar{u}_{\varepsilon} \otimes \bar{u}_{\varepsilon}}\right)=\widehat{g_{\delta}}(\xi) \mathcal{F}\left(\overline{u_{\varepsilon} \otimes u_{\varepsilon}}\right)=e^{-\frac{\delta^{2}}{4 \gamma}|\xi|^{2}}\left(\widehat{\bar{u}}_{\varepsilon} * \widehat{\bar{u}_{\varepsilon}}\right)
$$

Using a Taylor series approximation of the exponential, we can write the asymptotic development

$$
\mathcal{F}\left(\overline{\bar{u}_{\varepsilon} \otimes \bar{u}_{\varepsilon}}\right)=\left(1-\frac{\delta^{2}}{4 \gamma}|\xi|^{2}\right)\left(\widehat{\bar{u}_{\varepsilon}} * \widehat{u_{\varepsilon}}\right)+O\left(\delta^{4}\right), \quad \text { as } \delta \rightarrow 0 .
$$

Applying the same treatment to the three other terms in the right-hand side of (2.13), taking the inverse Fourier transform and neglecting every term of order $O\left(\delta^{4}\right)$ or higher, one obtains the approximation

$$
\overline{u_{\varepsilon} \otimes u_{\varepsilon}} \approx \bar{u}_{\varepsilon} \otimes \bar{u}_{\varepsilon}+\frac{\delta^{2}}{2 \gamma} D \bar{u}_{\varepsilon}\left(D \bar{u}_{\varepsilon}\right)^{T},
$$

where $D \bar{u}_{\varepsilon}$ stands for the Jacobian matrix of $\bar{u}_{\varepsilon}$. This leads to a classical space-filtered LES model that is used in many studies, see for instance $[1,5,6]$. However, following the previous approach, the turbulent fluctuations $\overline{u_{\varepsilon}^{\prime} \otimes u_{\varepsilon}^{\prime}}$ in (2.13) turn out to be formally of order $O\left(\delta^{4}\right)$, and thus neglictable. In the present work, we choose to model $\overline{u_{\varepsilon}^{\prime} \otimes u_{\varepsilon}^{\prime}}$ by a Smagorinsky term of the form $-C_{s}\left|D\left(\bar{u}_{\varepsilon}\right)\right| D\left(\bar{u}_{\varepsilon}\right)$, where $C_{s}>0$ is of order $O\left(\delta^{2}\right)$. This term was introduced in [42]. As a result, the model that we consider relies on the following approximation:

$$
\overline{u_{\varepsilon} \otimes u_{\varepsilon}} \approx \bar{u}_{\varepsilon} \otimes \bar{u}_{\varepsilon}+\frac{\delta^{2}}{2 \gamma} D \bar{u}_{\varepsilon}\left(D \bar{u}_{\varepsilon}\right)^{T}-C_{s}\left|D \bar{u}_{\varepsilon}\right| D \bar{u}_{\varepsilon}
$$

Defining $\left(w_{\varepsilon}, q_{\varepsilon}\right)$ as an approximation of $\left(\bar{u}_{\varepsilon}, \bar{p}_{\varepsilon}\right)$, we obtain the system

$$
\begin{aligned}
\partial_{t} w_{\varepsilon}+\left(w_{\varepsilon} \cdot \nabla\right) w_{\varepsilon}-\operatorname{div} S\left(D w_{\varepsilon}\right)+\nabla q_{\varepsilon} & =0 \quad \text { in }] 0, T\left[\times \Omega_{\varepsilon},\right. \\
\operatorname{div} w_{\varepsilon} & =0 \quad \text { in }] 0, T\left[\times \Omega_{\varepsilon},\right. \\
w_{\varepsilon}(x, 0) & =w_{\varepsilon}^{0}(x), \quad x \in \Omega_{\varepsilon},
\end{aligned}
$$

where the stress tensor $S\left(D w_{\varepsilon}\right)$ is defined by

$$
S\left(D w_{\varepsilon}\right)=\left(\frac{1}{R e}+C_{s}\left|D w_{\varepsilon}\right|\right) D w_{\varepsilon}-\frac{\delta^{2}}{2 \gamma} D w_{\varepsilon}\left(D w_{\varepsilon}\right)^{T} .
$$

In $(2.16)$, the initial datum $w_{\varepsilon}^{0}$ is obtained by setting $w_{\varepsilon}^{0}(x):=\overline{u_{\varepsilon}^{0}}(x)$ a.e. in $\Omega_{\varepsilon}$.

Treatment of the boundary conditions. To complete system (2.14)-(2.16), we need to impose boundary conditions. Formally, the approximation $w_{\varepsilon}$ should satisfy $w_{\varepsilon}=\bar{u}_{\varepsilon}$ on the boundary of the domain, so we need to replace such boundary conditions by suitable approximations in order to close the system. Since we want to focus on the interaction between the fluid and the rough boundary $\Gamma_{\varepsilon}$, for simplicity, we impose no-slip boundary condition on the top boundary

$$
w_{\varepsilon}=0 \quad \text { on } \Gamma^{0} \text {. }
$$


To derive a boundary condition for $w_{\varepsilon}$ on the rough plate $\Gamma_{\varepsilon}$, we follow a strategy proposed by Galdi and Layton [24]. In their paper, these authors suggest that the averaged velocity field, which is a model for the motion of the so-called large eddies, should satisfy a Navier slip boundary condition on solid boundaries, which can be seen as the most general boundary condition for a fluid. Some numerical evidence of this fact are cited by Serrin in [41], for high Reynolds flows of gases. In the context of the nonlinear stress tensor (2.17), such boundary conditions read

$$
\begin{gathered}
w_{\varepsilon} \cdot \nu=0 \text { on } \Gamma_{\varepsilon} \\
{\left[S\left(D w_{\varepsilon}\right) \nu\right] \cdot \tau_{i}+\beta w_{\varepsilon} \cdot \tau_{i}=0 \text { on } \Gamma_{\varepsilon} \quad i=1,2 .}
\end{gathered}
$$

In these expressions, $\nu$ denotes the outward unit vector on $\Gamma_{\varepsilon},\left\{\tau_{1}, \tau_{2}\right\}$ is an orthonormal system of tangent vectors on $\Gamma_{\varepsilon}$ and $\beta=\beta(R e, \delta)$ is a nonnegative friction coefficient, that depends typically on the Reynolds number $R e$ and on the averaging radius $\delta$. In particular, for consistency, the model should impose that $\beta$ goes to infinity as $\delta$ goes to 0 , so that formally, boundary conditions (2.19) turn into no slip conditions as the averaging radius vanishes, conformly to the physical boundary conditions (2.9) satisfied by the fluid.

In the present study, we make the assumption that, $\delta$ and $R e$ being fixed, $\beta$ remains of order 1 with respect to the roughness parameter $\varepsilon$, as $\varepsilon$ tends to zero. Thus, we assume for simplicity that $\beta(R e, \delta)$ is independant on $\varepsilon$. As it will be clear in the proofs, as long as the friction coefficient remains bounded when $\varepsilon$ goes to zero, it does not play any significant role in the asymptotic analysis of system (2.14)-(2.19).

Remark 2.1. In the computations, we will often divide the tensor $S\left(D w_{\varepsilon}\right)$ in two parts: the Newtonian and non-Newtonian parts. Namely, we set

$$
S\left(D w_{\varepsilon}\right)=\underbrace{\frac{1}{R e} D w_{\varepsilon}}_{\text {Newtonian part }}+\underbrace{\widehat{S}\left(D w_{\varepsilon}\right)}_{\text {non-Newtonian part }},
$$

with

$$
\widehat{S}\left(D w_{\varepsilon}\right)=C_{s}\left|D w_{\varepsilon}\right| D w_{\varepsilon}-\frac{\delta^{2}}{2 \gamma} D w_{\varepsilon}\left(D w_{\varepsilon}\right)^{T}
$$

Remark 2.2. Tensor $S(\xi)$ satisfies the homogeneity condition

$$
S(t \xi)=\frac{t}{R e} \xi+t^{2} \widehat{S}(\xi), \quad \forall \xi \in \mathbb{R}^{3 \times 3}, \quad \forall t \in \mathbb{R}_{+} .
$$

Weak formulation of system (2.14)-(2.19). We introduce the functional spaces

$$
\begin{aligned}
& H_{\varepsilon}=L^{2}\left(\Omega_{\varepsilon}, \mathbb{R}^{3}\right), \\
& W_{\varepsilon}=\left\{w \in W^{1,3}\left(\Omega_{\varepsilon}, \mathbb{R}^{3}\right), w \cdot \nu=0 \text { on } \Gamma_{\varepsilon}, w=0 \text { on } \Gamma^{0}\right\}, \\
& V_{\varepsilon}=\left\{w \in W_{\varepsilon}, \operatorname{div} w=0 \text { in } \Omega_{\varepsilon}\right\} .
\end{aligned}
$$

$W_{\varepsilon}$ and $V_{\varepsilon}$ are closed subspaces of $W^{1,3}\left(\Omega_{\varepsilon}, \mathbb{R}^{3}\right)$, and thus Banach spaces for the same norm $\|D w\|_{L^{3}\left(\Omega_{\varepsilon}, \mathbb{R}^{9}\right)}$, which is equivalent to the usual $W^{1,3}$ norm by Poincaré inequality. We define

$$
J_{\varepsilon}=H^{1}(] 0, T\left[, H_{\varepsilon}\right) \cap L^{3}(] 0, T\left[, V_{\varepsilon}\right) .
$$


Assume that $w_{\varepsilon}^{0} \in V_{\varepsilon}$ is given. We say that $w_{\varepsilon}$ is a weak solution to problem (2.14)-(2.19) if $w_{\varepsilon} \in J_{\varepsilon}$ and satisfies initial condition (2.16), and if the integral identity

$$
\begin{aligned}
& \int_{0}^{T} \int_{\Omega_{\varepsilon}} \partial_{t} w_{\varepsilon} \cdot \varphi d x d t+\int_{0}^{T} \int_{\Omega_{\varepsilon}}\left[\left(w_{\varepsilon} \cdot \nabla\right) w_{\varepsilon}\right] \cdot \varphi d x d t+\int_{0}^{T} \int_{\Omega_{\varepsilon}} S\left(D w_{\varepsilon}\right): D \varphi d x d t \\
& +\beta \int_{0}^{T} \int_{\Gamma_{\varepsilon}} w_{\varepsilon} \cdot \varphi d \mathcal{H}^{2} d t=\int_{0}^{T} \int_{\Omega_{\varepsilon}} \bar{f} \cdot \varphi d x d t
\end{aligned}
$$

holds for every test function $\varphi$ such that

$$
\varphi \in C_{c}^{1}(] 0, T\left[\times \overline{\Omega_{\varepsilon}}, \mathbb{R}^{3}\right), \operatorname{div} \varphi=0 \text { in } \Omega_{\varepsilon}, \varphi \cdot \nu=0 \text { on } \Gamma_{\varepsilon}, \varphi=0 \text { on } \Gamma^{0} .
$$

In the surfacic integral on $\Gamma_{\varepsilon}, \mathcal{H}^{2}$ stands for the bidimensional Hausdorff measure in $\mathbb{R}^{3}$. Notice that, by Sobolev embedding theorem, the space $J_{\varepsilon}$ is continuously embedded in $C^{0}\left([0, T], H_{\varepsilon}\right)$, so that initial condition (2.16) is well defined in $H_{\varepsilon}$.

Our main objective is to study the asymptotic behaviour of weak solutions to system (2.14)-(2.19) when $\varepsilon$ tends to zero, and to identify the homogenized model that accounts for the roughness induced effects.

\section{Main results}

The first result ensures the existence and uniqueness of a weak solution $w_{\varepsilon} \in J_{\varepsilon}$ to problem (2.23), and states the expected energy estimates. It is adapted from Coletti [19].

Theorem 3.1. Let $T>0$ and $\bar{f} \in H^{1}(] 0, T\left[, L^{2}\left(\Lambda, \mathbb{R}^{3}\right)\right)$. Assume that $C_{s}>\frac{\delta^{2}}{\gamma}$. Then for every $\varepsilon>0$ and every initial datum $w_{\varepsilon}^{0} \in V_{\varepsilon}$, there exists a unique weak solution $w_{\varepsilon} \in J_{\varepsilon}$ to problem (2.14)(2.19). Moreover, there exists a constant $C=C\left(R e, C_{s}, \delta, \gamma\right)$ such that this solution satisfies the energy inequalities:

$$
\begin{aligned}
& \sup _{0 \leq t \leq T}\left(\int_{\Omega_{\varepsilon}}\left|w_{\varepsilon}\right|^{2} d x\right) \leq \int_{\Omega_{\varepsilon}}\left|w_{\varepsilon}^{0}\right|^{2} d x+C \int_{0}^{T} \int_{\Omega_{\varepsilon}}|\bar{f}|^{2} d x d t, \\
& \frac{1}{R e} \int_{0}^{T} \int_{\Omega_{\varepsilon}}\left|D w_{\varepsilon}\right|^{2} d x d t+2 \int_{0}^{T} \int_{\Gamma_{\varepsilon}} \beta\left|w_{\varepsilon}\right|^{2} d \mathcal{H}^{2} d t+2\left(C_{s}-\frac{\delta^{2}}{2 \gamma}\right) \int_{0}^{T} \int_{\Omega_{\varepsilon}}\left|D w_{\varepsilon}\right|^{3} d x d t \\
& \leq \int_{\Omega_{\varepsilon}}\left|w_{\varepsilon}^{0}\right|^{2} d x+C \int_{0}^{T} \int_{\Omega_{\varepsilon}}|\bar{f}|^{2} d x d t, \\
& \int_{0}^{T} \int_{\Gamma_{\varepsilon}}\left|\partial_{t} w_{\varepsilon}\right|^{2} d \sigma d t \\
& \leq C\left(\int_{\Omega_{\varepsilon}}\left|w_{\varepsilon}^{0}\right|^{2} d x+\int_{\Omega_{\varepsilon}}\left|D w_{\varepsilon}^{0}\right|^{2} d x+\int_{\Omega_{\varepsilon}}\left|D w_{\varepsilon}^{0}\right|^{3} d x+\int_{0}^{T} \int_{\Omega_{\varepsilon}}|\bar{f}|^{2} d x d t+\int_{0}^{T} \int_{\Omega_{\varepsilon}}\left|\partial_{t} \bar{f}\right|^{2} d x d t\right) .
\end{aligned}
$$

In the next Proposition, we construct a pressure field $q_{\varepsilon} \in W^{-1, \infty}(] 0, T\left[, L_{0}^{3 / 2}\left(\Omega_{\varepsilon}\right)\right)$, associated with the divergence free constraint on $w_{\varepsilon}$. 
Proposition 3.2. Let $T>0, \bar{f} \in H^{1}(] 0, T\left[, L^{2}\left(\Lambda, \mathbb{R}^{3}\right)\right)$ and assume that $C_{s}>\frac{\delta^{2}}{\gamma}$. Let $\varepsilon>0, w_{\varepsilon}^{0} \in V_{\varepsilon}$ and let $w_{\varepsilon} \in J_{\varepsilon}$ be the weak solution to problem (2.14)-(2.19). Then, there exists a distribution $q_{\varepsilon} \in$ $W^{-1, \infty}(] 0, T\left[, L_{0}^{3 / 2}\left(\Omega_{\varepsilon}\right)\right)$ such that for every $\varphi \in C_{c}^{1}(] 0, T\left[, W_{\varepsilon}\right)$,

$$
\begin{aligned}
& \int_{0}^{T} \int_{\Omega_{\varepsilon}} \partial_{t} w_{\varepsilon} \cdot \varphi d x d t+\int_{0}^{T} \int_{\Omega_{\varepsilon}}\left[\left(w_{\varepsilon} \cdot \nabla\right) w_{\varepsilon}\right] \cdot \varphi d x d t+\int_{0}^{T} \int_{\Omega_{\varepsilon}} S\left(D w_{\varepsilon}\right): D \varphi d x d t \\
& +\beta \int_{0}^{T} \int_{\Gamma_{\varepsilon}} w_{\varepsilon} \cdot \varphi d \mathcal{H}^{2} d t-\int_{0}^{T} \int_{\Omega_{\varepsilon}} q_{\varepsilon} \operatorname{div} \varphi d x d t=\int_{0}^{T} \int_{\Omega_{\varepsilon}} \bar{f} \cdot \varphi d x d t .
\end{aligned}
$$

Our main contribution is the obtention of the asymptotic behaviour of the velocity/pressure couple $\left(w_{\varepsilon}, q_{\varepsilon}\right)$ satisfying $(3.4)$, as $\varepsilon$ tends to zero. The statement of the effective system requires some extra notation to define the auxiliary (cell) problem, that we introduce in the next paragraph.

Notation related to the cell problem. By $Y^{\prime}$, we denote the unitary cube of $\mathbb{R}^{2}, Y^{\prime}=\left(-\frac{1}{2}, \frac{1}{2}\right)^{2}$, and by $\widehat{Q}$ the set $\widehat{Q}=Y^{\prime} \times(0,+\infty)$. For every $M>0$ we write $\widehat{Q}_{M}=Y^{\prime} \times(0, M)$. We use the index \# to mean periodicity with respect $Y^{\prime}$, for example $L_{\#}^{p}\left(Y^{\prime}\right)$ denotes the space of functions $u \in L_{l o c}^{p}\left(\mathbb{R}^{2}\right)$ which are $Y^{\prime}$-periodic, while $L_{\#}^{p}(\widehat{Q})$ denotes the space of functions $\widehat{u} \in L_{l o c}^{p}\left(\mathbb{R}^{2} \times(0,+\infty)\right)$ such that

$$
\int_{\widehat{Q}}|\widehat{u}|^{p} d y<+\infty, \quad \widehat{u}\left(y^{\prime}+k^{\prime}, y_{3}\right)=\widehat{u}(y), \quad \forall k^{\prime} \in \mathbb{Z}^{2}, \quad \text { a.e. } y \in \mathbb{R}^{2} \times(0,+\infty) .
$$

We denote by $\mathcal{V}$ the space of functions $\widehat{v}: \mathbb{R}^{2} \times(0,+\infty) \rightarrow \mathbb{R}$ such that

$$
\forall M>0 \quad \widehat{v} \in W_{\#}^{1,3}\left(\widehat{Q}_{M}, \mathbb{R}\right), \quad \text { and } \quad D \widehat{v} \in L_{\#}^{3}\left(\widehat{Q}, \mathbb{R}^{3}\right) .
$$

It is a Banach space endowed with the norm $\|\cdot\|_{\mathcal{V}}$ defined by

$$
\|\widehat{v}\|_{\mathcal{V}}^{3}=\|\widehat{v}\|_{L_{\#}^{3}\left(Y^{\prime} \times\{0\}, \mathbb{R}\right)}^{3}+\|D \widehat{v}\|_{L_{\#}^{3}\left(\widehat{Q}, \mathbb{R}^{3}\right)}^{3} .
$$

Asymptotic behaviour of $\left(w_{\varepsilon}, q_{\varepsilon}\right)$. Depending on the scaling of the amplitude $a_{\varepsilon}$ with respect to $\varepsilon$, the asymptotic behaviour of system (2.14)-(2.19) is described by three different regimes, that we call critical, sub-critical and super-critical. The relevant scaling is controlled by parameter $\lambda$, which is a nonnegative number, possibly infinite, defined by

$$
\lambda:=\lim _{\varepsilon \rightarrow 0} \frac{a_{\varepsilon}}{\varepsilon^{\frac{5}{3}}} .
$$

Here, we state the result in the critical case; the other cases will be discussed in a Remark 3.7.

Theorem 3.3. Let $T>0, \bar{f} \in H^{1}(] 0, T\left[, L^{2}\left(\Lambda, \mathbb{R}^{3}\right)\right)$ and assume that $C_{s}>\frac{\delta^{2}}{\gamma}$. We consider a family of initial data $w_{\varepsilon}^{0} \in V_{\varepsilon}$, satisfying the uniform bound

$$
\forall \varepsilon>0 \quad\left\|w_{\varepsilon}^{0}\right\|_{W_{\varepsilon}} \leq C,
$$

where $C>0$ is a constant. Without loss of generality, we may assume the existence of $w^{0} \in W^{1,3}\left(\Omega, \mathbb{R}^{3}\right)$ such that $w_{\varepsilon \mid \Omega}^{0}$ converges weakly in $W^{1,3}\left(\Omega, \mathbb{R}^{3}\right)$, to $w^{0}$.

For every $\varepsilon>0$, let $w_{\varepsilon} \in J_{\varepsilon}$ be the weak solution to problem $(2.14)-(2.19)$ and $q_{\varepsilon} \in W^{-1, \infty}(] 0, T\left[, L_{0}^{3 / 2}\left(\Omega_{\varepsilon}\right)\right)$ be the associated pressure. Assume $a_{\varepsilon} \approx \varepsilon^{\frac{5}{3}}$ with $a_{\varepsilon} / \varepsilon^{\frac{5}{3}} \rightarrow \lambda, 0<\lambda<+\infty$. Then, there exists $(w, q) \in L^{3}(] 0, T\left[, W^{1,3}\left(\Omega, \mathbb{R}^{3}\right)\right) \times W^{-1, \infty}(] 0, T\left[, L_{0}^{\frac{3}{2}}(\Omega)\right)$ such that

$$
w_{\varepsilon \mid \Omega} \rightarrow w \quad \text { in } L^{3}(] 0, T\left[, W^{1,3}\left(\Omega, \mathbb{R}^{3}\right)\right), \quad q_{\varepsilon \mid \Omega} \rightarrow q \quad \text { in } W^{-1, \infty}(] 0, T\left[, L^{\frac{3}{2}}(\Omega)\right),
$$


where $(w, q)$ satisfies the effective problem

$$
\left\{\begin{aligned}
\partial_{t} w+(w \cdot \nabla) w-\operatorname{div}(S(D w))+\nabla q & =\bar{f} \quad \text { in }] 0, T[\times \Omega, \\
\operatorname{div} w & =0 \quad \text { in }] 0, T[\times \Omega, \\
w(0, x) & =w^{0}(x), \quad x \in \Omega, \\
w & =0 \quad \text { on }] 0, T\left[\times \Gamma^{0},\right.
\end{aligned}\right.
$$

plus the effective boundary condition at the bottom

$$
\begin{aligned}
w_{3}=0 & \text { on }] 0, T[\times \Gamma, \\
-S(D w)_{1,3}+\beta w_{1}+\lambda^{3} R_{1}\left(w^{\prime}\right)=0 & \text { on }] 0, T[\times \Gamma, \\
-S(D w)_{2,3}+\beta w_{2}+\lambda^{3} R_{2}\left(w^{\prime}\right)=0 & \text { on }] 0, T[\times \Gamma .
\end{aligned}
$$

Here, $R_{i}: \mathbb{R}^{2} \rightarrow \mathbb{R}, \xi^{\prime} \mapsto R_{i}, i \in\{1,2\}$, are defined by

$$
R_{i}\left(\xi^{\prime}\right)=\int_{\widehat{Q}} \widehat{S}\left(D_{y} \widehat{\phi}^{\xi^{\prime}}\right): D_{y} \widehat{\phi}^{e_{i}} d y \quad \forall \xi^{\prime} \in \mathbb{R}^{2}
$$

where for every $\xi^{\prime} \in \mathbb{R}^{2},\left(\widehat{\phi}^{\xi^{\prime}}, \widehat{\pi}^{\xi^{\prime}}\right) \in(\mathcal{V} / \mathbb{R})^{2} \times \mathcal{V} \times L_{\#}^{\frac{3}{2}}(\widehat{Q}, \mathbb{R})$ is the solution of

$$
\left\{\begin{array}{l}
-\operatorname{div}_{\mathrm{y}}\left(\widehat{S}\left(D_{y} \widehat{\phi}^{\xi^{\prime}}\right)\right)+\nabla_{y} \widehat{\pi}^{\xi^{\prime}}=0 \quad \text { in } \mathcal{T}^{2} \times \mathbb{R}^{+}, \\
\operatorname{div}_{\mathrm{y}} \widehat{\phi}^{\xi^{\prime}}=0 \quad \text { in } \mathcal{T}^{2} \times \mathbb{R}^{+}, \\
\widehat{\phi}_{3}^{\xi^{\prime}}\left(y^{\prime}, 0\right)=-\nabla \Psi\left(y^{\prime}\right) \cdot \xi^{\prime} \text { on } \mathcal{T}^{2} \times\{0\}, \\
\widehat{S}\left(D_{y} \widehat{\phi}^{\xi^{\prime}}\right)_{i, 3}=0, i=1,2, \quad \text { on } \mathcal{T}^{2} \times\{0\} .
\end{array}\right.
$$

Remark 3.4. The roughness effect depends only on the non-Newtonian part $\widehat{S}$ of the stress tensor, that describes the large-scale/small-scale interactions in Smagorinsky's LES model, and of the geometry of the riblets, specified by the function $\Psi$ whose gradient determines the normal boundary condition in boundary layer system (3.10).

Remark 3.5. Theorem 3.3 can be easily adapted to cover the two other asymptotic regimes.

- In the sub-critical case $a_{\varepsilon} / \varepsilon^{\frac{5}{3}} \rightarrow 0$, the result holds replacing $\lambda$ by 0 in conditions (3.8). In this regime, the roughness has no effect on the boundary condition satisfied by the homogenized velocity field $w$.

- In the super-critical case $a_{\varepsilon} / \varepsilon^{\frac{5}{3}} \rightarrow+\infty$, boundary conditions (3.8) are replaced by no-slip conditions $w=0$ on $] 0, T[\times \Gamma$. In that case, the roughness effect is maximal.

The particular case of riblets. In a wide variety of applications to fluid mechanics, rough boundaries are composed of ribbed walls. In that case, the geometry of the rough surface presents a direction of invariance, which be modeled by considering a function $\Psi_{\varepsilon}$ of the form $\Psi_{\varepsilon}\left(x^{\prime}\right)=a_{\varepsilon} \Psi\left(x_{1} / \varepsilon\right)$, where $\Psi$ is a positive one-variable function, periodic with period 1. In this particular setting, the description of the rugosity effect can be simplified, which leads to the following result. 
Corollary 3.6. Under the same hypotheses as in Theorem 3.3, if we assume in addition that the sequence of rough walls $\Gamma_{\varepsilon}$ is described by functions $\Psi_{\varepsilon}$ of the form

$$
\Psi_{\varepsilon}\left(x^{\prime}\right)=a_{\varepsilon} \Psi\left(x_{1} / \varepsilon\right), \quad x^{\prime} \in \mathcal{T}^{2},
$$

where $\Psi$ is a given positive 1-periodic function of variable $x_{1}$, then boundary conditions (3.8) are replaced by the following ones:

$$
\begin{aligned}
w_{3}=0 & \text { on }] 0, T[\times \Gamma, \\
-S(D w)_{1,3}+\beta w_{1}+\lambda^{3} w_{1}^{2} R_{1}\left(\operatorname{sgn}\left(w_{1}\right) e_{1}\right)=0 & \text { on }] 0, T[\times \Gamma, \\
-S(D w)_{2,3}+\beta w_{2}=0 & \text { on }] 0, T[\times \Gamma,
\end{aligned}
$$

where $\operatorname{sgn}$ is the sign function $\operatorname{sgn}(0)=0, \operatorname{sgn}(s)=\frac{s}{|s|}$ if $s \in \mathbb{R} \backslash\{0\}$, and $R_{1}(\cdot)$ is given by (3.9).

Remark 3.7. Observe that in the case of straight riblets, the roughness effect occurs only in the transverse direction $e_{1}$, whereas the boundary condition along $e_{2}$ (the direction of the riblets) is preserved. Besides, contrary to the general case, the expression of the new interaction term $\lambda^{3} w_{1}^{2} R_{1}\left(\operatorname{sgn}\left(w_{1}\right) e_{1}\right)$ involves only the first component $w_{1}$ of the fluid velocity.

Finally, Corollary 3.6 can be easily adapted to describe the two other asymptotic regimes.

- In the sub-critical case $a_{\varepsilon} / \varepsilon^{\frac{5}{3}} \rightarrow 0$, the result holds replacing $\lambda$ by 0 in conditions (3.11). In that case, there is no roughness effect in both $e_{1}$ and $e_{2}$ directions.

- In the super-critical case $a_{\varepsilon} / \varepsilon^{\frac{5}{3}} \rightarrow+\infty$, the second boundary condition in (3.11) is replaced by condition $w_{1}=0$ on $] 0, T[\times \Gamma$. In that case, a no-slip condition appears in the transversal direction $e_{1}$, while there is no roughness effect in direction $e_{2}$.

The following proposition ensures the existence and uniqueness of the solution to system (3.10) and states some exponential decay properties in the $y_{3}$-direction that will be useful to perform numerical simulations.

Proposition 3.8. Assume that $C_{s}-\frac{\delta^{2}}{\gamma}>0$. For every $\xi^{\prime} \in \mathbb{R}^{2}$, there exists a unique solution $\left(\widehat{\phi}^{\xi^{\prime}}, \widehat{\pi}^{\xi^{\prime}}\right) \in$ $(\mathcal{V} / \mathbb{R})^{2} \times \mathcal{V} \times L_{\#}^{\frac{3}{2}}(\widehat{Q}, \mathbb{R})$ to system (3.10). Moreover, there exist constants $C, \tau>0$ such that, for every $z_{3}>0$,

$$
\begin{gathered}
\int_{\widehat{Q}}\left|D_{y} \widehat{\phi}^{\xi^{\prime}}\right|^{3} d y \leq C\left|\xi^{\prime}\right|^{3} \int_{\mathcal{T}^{2}}|\nabla \Psi|^{3} d y^{\prime} \\
\int_{\mathcal{T}^{2} \times\left(z_{3},+\infty\right)}\left|D_{y} \widehat{\phi}^{\xi^{\prime}}\right|^{3} d y \leq C\left|\xi^{\prime}\right|^{3} e^{-\tau z_{3}} \int_{\mathcal{T}^{2}}|\nabla \Psi|^{3} d y^{\prime} .
\end{gathered}
$$

\section{Proof of the main results}

Proof of Theorem 3.1. The proof of Theorem 3.1 is a straightforward adaptation of the proof by Coletti, who established in [19] the existence of a weak solution for the model (2.14)-(2.16), in the case of homogeneous Dirichlet boundary conditions on the boundary of a Lipschitz domain. As the addition of a friction term depending on $\beta$ does not affect the analysis of the system, we omit the proof. However, let us recall some crucial properties of the operator $\widehat{S}: \mathbb{R}^{3 \times 3} \rightarrow \mathbb{R}^{3 \times 3}$ (introduced in Remark 2.1) defined by

$$
\forall \zeta \in \mathbb{R}^{3 \times 3} \quad \widehat{S}(\zeta)=C_{s}|\zeta| \zeta-\frac{\delta^{2}}{2 \gamma} \zeta \zeta^{T}
$$


which are the essential ingredients of the proof by Coletti:

$$
\begin{aligned}
& \forall \zeta \in \mathbb{R}^{3 \times 3} \quad|\widehat{S}(\zeta)| \leq\left(C_{s}+\frac{\delta^{2}}{2 \gamma}\right)|\zeta|^{2}, \\
& \forall \zeta \in \mathbb{R}^{3 \times 3} \quad \widehat{S}(\zeta): \zeta \geq\left(C_{s}-\frac{\delta^{2}}{2 \gamma}\right)|\zeta|^{3}, \\
& \forall\left(w_{1}, w_{2}\right) \in W_{\varepsilon} \times W_{\varepsilon} \quad \int_{\Omega_{\varepsilon}}\left(\widehat{S}\left(D w_{1}\right)-\widehat{S}\left(D w_{2}\right)\right):\left(D w_{1}-D w_{2}\right) d x \geq 0 .
\end{aligned}
$$

In the energy inequalities (3.1)-(3.2), the uniformity of the constant $C$ with respect to $\varepsilon$ results from the existence of a uniform Poincaré inequality in the sequence of domains $\Omega_{\varepsilon}$.

Let $W_{\varepsilon}^{\prime}$ be the dual space of $W_{\varepsilon}$ (defined by $(2.21)$ ). To construct the pressure $q_{\varepsilon}$ associated with a weak solution $w_{\varepsilon}$ to problem (2.14)-(2.19), we rely on the following lemma, which is an adaptation of the result by Tartar [44] to the present setting where the duality $H^{-1}, H_{0}^{1}$ is replaced by $W_{\varepsilon}^{\prime}, W_{\varepsilon}$.

Lemma 4.1. The following properties are equivalent.

(P1) Let $G \in W_{\varepsilon}{ }^{\prime}$ such that for every $w \in V_{\varepsilon},\langle G, w\rangle_{W_{\varepsilon}{ }^{\prime}, W_{\varepsilon}}=0$. Then there exists $\pi_{\varepsilon} \in L^{3 / 2}\left(\Omega_{\varepsilon}\right)$ such that for every $\varphi \in W_{\varepsilon},\langle G, \varphi\rangle_{W_{\varepsilon}^{\prime}, W_{\varepsilon}}=-\int_{\Omega_{\varepsilon}} \pi_{\varepsilon} \operatorname{div} \varphi d x$.

(P2) For every $h \in L^{3}\left(\Omega_{\varepsilon}, \mathbb{R}\right)$ such that $\int_{\Omega_{\varepsilon}} h d x=0$, there exists $u \in W_{\varepsilon}$ such that $\operatorname{div} u=h$.

Proof of Lemma 4.1. We denote by $A: L^{3 / 2}\left(\Omega_{\varepsilon}, \mathbb{R}\right) \rightarrow W_{\varepsilon}{ }^{\prime}$ the linear operator defined by

$$
\forall(\varphi, \xi) \in L^{3 / 2}\left(\Omega_{\varepsilon}, \mathbb{R}\right) \times W_{\varepsilon} \quad\langle A \varphi, \xi\rangle_{W_{\varepsilon}{ }^{\prime}, W_{\varepsilon}}=-\int_{\Omega_{\varepsilon}} \varphi \operatorname{div} \xi d x .
$$

Denoting by $C$ a positive constant such that $\|\xi\|_{W_{\varepsilon}}=\|D \xi\|_{L^{3}\left(\Omega_{\varepsilon}, \mathbb{R}^{3}\right)} \geq C^{-1}\|\operatorname{div} \xi\|_{L^{3}\left(\Omega_{\varepsilon}\right)}$, using Hölder inequality we obtain that $A$ belongs to $\mathcal{L}\left(L^{3 / 2}\left(\Omega_{\varepsilon}\right), W_{\varepsilon}^{\prime}\right)$, with norm bounded by $C$.

Let $A^{T}: W_{\varepsilon} \rightarrow L^{3}\left(\Omega_{\varepsilon}\right)$ be the transpose of $A$. By definition, we have for every pair $(\varphi, \xi) \in$ $L^{3 / 2}\left(\Omega_{\varepsilon}\right) \times W_{\varepsilon},\left\langle\varphi, A^{T} \xi\right\rangle_{L^{3 / 2}\left(\Omega_{\varepsilon}\right), L^{3}\left(\Omega_{\varepsilon}\right)}=-\int_{\Omega_{\varepsilon}} \varphi \operatorname{div} \xi d x$, so that we can identify $A^{T} \xi$ with $-\operatorname{div} \xi$, and observe that $R\left(A^{T}\right) \subset L_{0}^{3}\left(\Omega_{\varepsilon}, \mathbb{R}\right)$. Consequently, the space $V_{\varepsilon}$ is the kernel of $A^{T}$, and if we denote by $R(A)$ the range of $A$, we have $\overline{R(A)}=V_{\varepsilon}^{\perp}$. Thus, property (P1) states that if a distribution $G$ belongs to $\overline{R(A)}$, then $G$ belong in fact to $R(A)$, since $G=A \pi_{\varepsilon}$ for a certain $\pi_{\varepsilon} \in L^{3 / 2}\left(\Omega_{\varepsilon}\right)$. As a result, property $(P 1)$ is equivalent to the fact that $R(A)$ is closed.

On the other hand, every function $\varphi \in \operatorname{Ker} A$ satisfies $\int_{\Omega_{\varepsilon}} \varphi \operatorname{div} \xi d x=0$ for any $\xi \in C_{c}^{\infty}\left(\Omega_{\varepsilon}, \mathbb{R}^{3}\right)$, so that $\nabla \varphi=0$ in the sense of distributions in $\Omega_{\varepsilon}$. Since $\Omega_{\varepsilon}$ is connected, $\varphi$ is a constant, so that the kernel of $A$ is generated by the constant function equal to 1 in $\Omega_{\varepsilon}$. This implies that $L_{0}^{3}\left(\Omega_{\varepsilon}\right)$ is the orthogonal of $\operatorname{Ker} A$ (in the $L^{3 / 2}, L^{3}$ duality). Moreover, by classical results on bounded linear operators between Banach spaces, we know that $\operatorname{Ker}(A)^{\perp}$ is the closure of $R\left(A^{T}\right)$ in $L^{3}\left(\Omega_{\varepsilon}\right)$.

Property (P2) can be rephrased as $L_{0}^{3}\left(\Omega_{\varepsilon}\right)=R\left(A^{T}\right)$, which is equivalent to $\overline{R\left(A^{T}\right)}=R\left(A^{T}\right)$, that is, $R\left(A^{T}\right)$ is closed in $L^{3}\left(\Omega_{\varepsilon}\right)$. Since $R(A)$ is closed if and only if $R\left(A^{T}\right)$ is closed (see for instance [11], Theorem II.18), we conclude that (P1) and (P2) are equivalent.

Now, we are in a position to prove Proposition 3.2 . 
Proof of Proposition 3.2. Let $C_{s}>\frac{\delta^{2}}{\gamma}, w_{\varepsilon}^{0} \in V_{\varepsilon}$ and $w_{\varepsilon} \in J_{\varepsilon}$ be a weak solution. Testing against $\varphi(t, x)=\eta(t) \phi(x)$ with $\eta \in C_{c}^{\infty}(] 0, T[)$ and $\phi \in V_{\varepsilon}$ in the weak formulation (2.23), we obtain for a.e. $t \in] 0, T[$,

$$
\begin{aligned}
& \int_{\Omega_{\varepsilon}} \partial_{t} w_{\varepsilon}(t) \cdot \phi d x+\int_{\Omega_{\varepsilon}}\left[\left(w_{\varepsilon}(t) \cdot \nabla\right) w_{\varepsilon}(t)\right] \cdot \phi d x+\int_{\Omega_{\varepsilon}} S\left(D w_{\varepsilon}(t)\right): D \phi d x \\
& +\beta \int_{\Gamma_{\varepsilon}} w_{\varepsilon}(t) \cdot \phi d \mathcal{H}^{2}-\int_{\Omega_{\varepsilon}} \bar{f} \cdot \phi d x=0 .
\end{aligned}
$$

Now, for every $t \in] 0, T$, we can integrate relation $(4.4)$ in $(0, t)$, to obtain

$$
\left\langle G_{\varepsilon}(t), \phi\right\rangle_{W_{\varepsilon}^{\prime}, W_{\varepsilon}}=0 \quad \forall \phi \in V_{\varepsilon}
$$

where $G_{\varepsilon}(t) \in W_{\varepsilon}^{\prime}$ is defined by:

$$
G_{\varepsilon}(t)=w_{\varepsilon}(t)-w_{\varepsilon}^{0}+\int_{0}^{t}\left(w_{\varepsilon}(\tau) \cdot \nabla\right) w_{\varepsilon}(\tau) d \tau+\int_{0}^{t} B_{\varepsilon}\left(w_{\varepsilon}(\tau)\right) d \tau-\int_{0}^{t} \bar{f}(\tau) d \tau
$$

In the above expression, the operator $B_{\varepsilon}: W_{\varepsilon} \rightarrow W_{\varepsilon}^{\prime}$ is given by

$$
\forall(v, \psi) \in W_{\varepsilon} \times W_{\varepsilon} \quad\left\langle B_{\varepsilon}(v), \psi\right\rangle_{W_{\varepsilon}{ }^{\prime}, W_{\varepsilon}}=\int_{\Omega_{\varepsilon}} S(D v): D \psi d x+\beta \int_{\Gamma_{\varepsilon}} v \cdot \psi d \mathcal{H}^{2}
$$

Since $\Gamma_{\varepsilon}$ is regular, property (P2) from Lemma 4.1 holds in $\Omega_{\varepsilon}$ (see for instance [23], Theorem 3.1 in Chap. III), so that we can apply (P1). Consequently, relation (4.5) yields the existence, for a.e. $t \in] 0, T[$, of $\pi_{\varepsilon}(t) \in L^{3 / 2}\left(\Omega_{\varepsilon}\right)$ such that

$$
\forall \phi \in W_{\varepsilon} \quad\langle G(t), \phi\rangle_{W_{\varepsilon}^{\prime}, W_{\varepsilon}}=-\int_{\Omega_{\varepsilon}} \pi_{\varepsilon}(t) \operatorname{div} \phi d x
$$

Without loss of generality, we can assume that for a.e. $t, \pi_{\varepsilon}(t) \in L_{0}^{3 / 2}\left(\Omega_{\varepsilon}\right)$.

Let us prove that the mapping $t \in] 0, T\left[\mapsto G_{\varepsilon}(t) \in W_{\varepsilon}^{\prime}\right.$ is continuous. Since $w_{\varepsilon}$ is in $H^{1}(] 0, T\left[, L^{2}\left(\Omega_{\varepsilon}, \mathbb{R}^{3}\right)\right)$, by Sobolev imbedding, $w_{\varepsilon}$ is continuous in time with values in $L^{2}\left(\Omega_{\varepsilon}, \mathbb{R}^{3}\right)$, and hence, continuous in time with values in $W_{\varepsilon}{ }^{\prime}$. Moreover, $\left(w_{\varepsilon} \cdot \nabla\right) w_{\varepsilon} \in L^{3 / 2}(] 0, T\left[, W_{\varepsilon}^{\prime}\right)$, due to the estimate

$$
\begin{aligned}
\int_{0}^{T}\left\langle\left(w_{\varepsilon} \cdot \nabla\right) w_{\varepsilon}, \varphi\right\rangle_{W_{\varepsilon}{ }^{\prime}, W_{\varepsilon}} d t & =\int_{0}^{T} \int_{\Omega_{\varepsilon}}\left[\left(w_{\varepsilon} \cdot \nabla\right) w_{\varepsilon}\right] \cdot \varphi d x d t \\
& =-\int_{0}^{T} \int_{\Omega_{\varepsilon}}\left[\left(w_{\varepsilon} \cdot \nabla\right) \varphi\right] \cdot w_{\varepsilon} d x d t \\
& \leq \int_{0}^{T}\left\|w_{\varepsilon}\right\|_{L^{3}\left(\Omega_{\varepsilon}, \mathbb{R}^{3}\right)}^{2}\|\nabla \varphi\|_{L^{3}\left(\Omega_{\varepsilon}, \mathbb{R}^{3}\right)} d t \\
& \leq\left(\int_{0}^{T}\left\|w_{\varepsilon}\right\|_{L^{3}\left(\Omega_{\varepsilon}, \mathbb{R}^{3}\right)}^{3} d t\right)^{2 / 3}\left(\int_{0}^{T}\|\nabla \varphi\|_{L^{3}\left(\Omega_{\varepsilon}, \mathbb{R}^{3}\right)}^{3} d t\right)^{1 / 3} \\
& =\left\|w_{\varepsilon}\right\|_{L^{3}(] 0, T\left[\times \Omega_{\varepsilon}, \mathbb{R}^{3}\right)}^{2}\|\varphi\|_{L^{3}(] 0, T\left[W_{\varepsilon}\right)},
\end{aligned}
$$


which holds for every $\varphi \in L^{3}(] 0, T\left[, W_{\varepsilon}\right)$. Similarly,

$$
\begin{aligned}
\frac{1}{R e} \int_{0}^{T} \int_{\Omega_{\varepsilon}} D w_{\varepsilon}: D \varphi d x d t & \leq \frac{1}{R e}\left\|D w_{\varepsilon}\right\|_{L^{2}(] 0, T\left[\times \Omega_{\varepsilon}, \mathbb{R}^{9}\right)}\|D \varphi\|_{L^{2}(] 0, T\left[\times \Omega_{\varepsilon}, \mathbb{R}^{9}\right)} \\
& \leq C\left\|D w_{\varepsilon}\right\|_{L^{3}(] 0, T\left[\times \Omega_{\varepsilon}, \mathbb{R}^{9}\right)}\|D \varphi\|_{L^{3}(] 0, T\left[\times \Omega_{\varepsilon}, \mathbb{R}^{9}\right)} \\
& \leq C\left\|w_{\varepsilon}\right\|_{L^{3}(] 0, T\left[, W_{\varepsilon}\right)}\|\varphi\|_{L^{3}(] 0, T\left[, W_{\varepsilon}\right)} \\
\beta \int_{0}^{T} \int_{\Gamma_{\varepsilon}} w_{\varepsilon} \cdot \varphi d x d t & \leq \beta \int_{0}^{T}\left\|w_{\varepsilon}\right\|_{L^{2}\left(\Gamma_{\varepsilon}, \mathbb{R}^{3}\right)}\|\varphi\|_{L^{2}\left(\Gamma_{\varepsilon}, \mathbb{R}^{3}\right)} \\
& \leq C\left(\int_{0}^{T}\left\|D w_{\varepsilon}\right\|_{L^{2}\left(\Omega_{\varepsilon}, \mathbb{R}^{9}\right)}^{2} d t\right)^{1 / 2}\left(\int_{0}^{T}\|D \varphi\|_{L^{2}\left(\Omega_{\varepsilon}, \mathbb{R}^{9}\right)}^{2} d t\right)^{1 / 2} \\
& \leq C\left\|D w_{\varepsilon}\right\|_{L^{2}(] 0, T\left[\times \Omega_{\varepsilon}, \mathbb{R}^{9}\right)}\|D \varphi\|_{L^{2}(] 0, T\left[\times \Omega_{\varepsilon}, \mathbb{R}^{9}\right)} \\
& \leq C\left\|D w_{\varepsilon}\right\|_{L^{3}(] 0, T\left[\times \Omega_{\varepsilon}, \mathbb{R}^{9}\right)}\|D \varphi\|_{L^{3}(] 0, T\left[\times \Omega_{\varepsilon}, \mathbb{R}^{9}\right)} \\
& \leq C\left\|w_{\varepsilon}\right\|_{L^{3}(] 0, T\left[, W_{\varepsilon}\right)}\|\varphi\|_{L^{3}(] 0, T\left[, W_{\varepsilon}\right)} \\
\int_{0}^{T} \int_{\Omega_{\varepsilon}} \widehat{S}\left(D w_{\varepsilon}\right): D \varphi d x d t & \leq \int_{0}^{T}\left\|D w_{\varepsilon}\right\|_{L^{3}\left(\Omega_{\varepsilon}, \mathbb{R}^{9}\right)}^{2}\|D \varphi\|_{L^{3}\left(\Omega_{\varepsilon}, \mathbb{R}^{9}\right)} d t \\
& \leq\left(\int_{0}^{T}\left\|D w_{\varepsilon}\right\|_{L^{3}\left(\Omega_{\varepsilon}, \mathbb{R}^{9}\right)}^{3} d t\right)^{2 / 3}\left(\int_{0}^{T}\|D \varphi\|_{L^{3}\left(\Omega_{\varepsilon}, \mathbb{R}^{9}\right)}^{3} d t\right)^{1 / 3} \\
& \leq\left\|D w_{\varepsilon}\right\|_{L^{3}(] 0, T\left[\times \Omega_{\varepsilon}, \mathbb{R}^{9}\right)}^{2}\|D \varphi\|_{L^{3}(] 0, T\left[\times \Omega_{\varepsilon}, \mathbb{R}^{9}\right)} \\
& \leq\left\|w_{\varepsilon}\right\|_{L^{3}(] 0, T\left[, W_{\varepsilon}\right)}^{2}\|\varphi\|_{L^{3}(] 0, T\left[, W_{\varepsilon}\right)}
\end{aligned}
$$

Thus, $T\left(w_{\varepsilon}\right) \in L^{3 / 2}(] 0, T\left[, W_{\varepsilon}^{\prime}\right)$. Finally, using Hölder inequality and the estimate $\|\varphi\|_{L^{2}(] 0, T\left[\times \Omega_{\varepsilon}, \mathbb{R}^{3}\right)} \leq$ $C\|\varphi\|_{L^{3}(] 0, T\left[, W_{\varepsilon}\right)}$, it is easy to see that $\bar{f} \in L^{3 / 2}(] 0, T\left[, W_{\varepsilon}^{\prime}\right)$.

Gathering the previous results, we obtain that $G-w_{\varepsilon}-w_{\varepsilon}^{0}$ is the antiderivative of the sum of integrable functions with values in $W_{\varepsilon}^{\prime}$. Since $w_{\varepsilon}$ is continuous in time, this implies that $G$ is continuous with values in $W_{\varepsilon}{ }^{\prime}$. Using 4.7 , we deduce that for every $\phi \in L^{3}(] 0, T\left[, W_{\varepsilon}\right)$, the mapping $\left.t \in\right] 0, T\left[\mapsto \int_{\Omega_{\varepsilon}} \pi_{\varepsilon}(t) \operatorname{div} \phi d x\right.$ is continuous. Hence, the mapping $t \in] 0, T\left[\mapsto \pi_{\varepsilon}(t) \in L_{0}^{3 / 2}\left(\Omega_{\varepsilon}\right)\right.$ is weakly continuous. Indeed, let $m \in L^{3}\left(\Omega_{\varepsilon}\right)$. By property $(P 2)$, there exists $h \in W_{\varepsilon}$ such that $\operatorname{div} h=m-\frac{1}{\left|\Omega_{\varepsilon}\right|} \int_{\Omega_{\varepsilon}} m d x$. Since for a.e. $t$, $\pi_{\varepsilon}(t)$ has zero average, there holds for a.e. $\left.t \in\right] 0, T[$

$$
\begin{aligned}
\langle\pi(t), m\rangle_{L^{3 / 2}\left(\Omega_{\varepsilon}\right), L^{3}\left(\Omega_{\varepsilon}\right)} & =\int_{\Omega_{\varepsilon}} \pi_{\varepsilon}(t)\left[m-\frac{1}{\left|\Omega_{\varepsilon}\right|} \int_{\Omega_{\varepsilon}} m d x\right] d x \\
& =\int_{\Omega_{\varepsilon}} \pi_{\varepsilon}(t) \operatorname{div} h d x \\
& =-\langle G(t), h\rangle_{W_{\varepsilon}{ }^{\prime}, W_{\varepsilon}} .
\end{aligned}
$$

Moreover, there exists a constant $C$ such that

$$
\begin{aligned}
\|h\|_{W_{\varepsilon}} & \leq C\left\|m-\frac{1}{\left|\Omega_{\varepsilon}\right|} \int_{\Omega_{\varepsilon}} m d x\right\|_{L^{3}\left(\Omega_{\varepsilon}\right)} \\
& \leq C\|m\|_{L^{3}\left(\Omega_{\varepsilon}\right)}
\end{aligned}
$$

From (4.12), we deduce

$$
\left.\left\|\pi_{\varepsilon}(t)\right\|_{L^{3 / 2}\left(\Omega_{\varepsilon}\right)} \leq C\left\|G_{\varepsilon}(t)\right\|_{W_{\varepsilon}{ }^{\prime}} \quad \text { for a.e. } t \in\right] 0, T[\text {. }
$$


By definition of $G_{\varepsilon}$, using similar estimates as (4.8)-(4.11), we conclude that there exists a constant $C>0$, such that

$$
\left\|\pi_{\varepsilon}\right\|_{L^{\infty}(] 0, T\left[, L_{0}^{3 / 2}\left(\Omega_{\varepsilon}\right)\right)} \leq C .
$$

Now, we can define $q_{\varepsilon} \in W^{-1, \infty}(] 0, T\left[, L_{0}^{3 / 2}\left(\Omega_{\varepsilon}\right)\right)$ by $q_{\varepsilon}=-\frac{\partial \pi}{\partial t}$, and $q_{\varepsilon}$ satisfies the corresponding estimate

$$
\left\|q_{\varepsilon}\right\|_{W^{-1, \infty}(] 0, T\left[, L_{0}^{3 / 2}\left(\Omega_{\varepsilon}\right)\right)} \leq C .
$$

Finally, to obtain relation (3.4), we consider $\varphi \in C_{c}^{\infty}(] 0, T\left[, W_{\varepsilon}\right)$ and for any $\left.t \in\right] 0, T$, we test against $\partial_{t} \varphi$ in (4.7). This yields

$$
\begin{aligned}
\forall t \in] 0, T[ & \int_{\Omega_{\varepsilon}}\left(w_{\varepsilon}(t)-w_{\varepsilon}^{0}\right) \cdot \partial_{t} \varphi d x+\int_{0}^{t} \int_{\Omega_{\varepsilon}}\left[\left(w_{\varepsilon}(\tau) \cdot \nabla\right) w_{\varepsilon}(\tau)\right] \cdot \partial_{t} \varphi d x d \tau \\
& +\int_{0}^{t}\left(\int_{\Omega_{\varepsilon}} S\left(D w_{\varepsilon}(\tau)\right): D\left(\partial_{t} \varphi\right) d x+\beta \int_{\Gamma_{\varepsilon}} w_{\varepsilon}(\tau) \cdot \partial_{t} \varphi d \mathcal{H}^{2}\right) d \tau \\
& -\int_{0}^{t}\left\langle\bar{f}(\tau), \partial_{t} \varphi\right\rangle_{W_{\varepsilon}{ }^{\prime}, W_{\varepsilon}} d \tau=-\int_{\Omega_{\varepsilon}} \pi_{\varepsilon}(t) \operatorname{div}\left(\partial_{t} \varphi\right) d x
\end{aligned}
$$

Commuting the integrations on $\Omega_{\varepsilon}$ with the derivative in time, this can be rewritten as: for a.e. $\left.t \in\right] 0, T[$,

$$
\begin{array}{r}
\int_{\Omega_{\varepsilon}}\left(w_{\varepsilon}(t)-w_{\varepsilon}^{0}\right) \cdot \partial_{t} \varphi d x+\int_{\Omega_{\varepsilon}}\left[w_{\varepsilon}(t) \cdot \nabla w_{\varepsilon}(t)\right] \cdot \varphi d x \\
\quad+\int_{\Omega_{\varepsilon}} S\left(D w_{\varepsilon}(t)\right): D \varphi d x+\beta \int_{\Gamma_{\varepsilon}} w_{\varepsilon}(t) \cdot \varphi d \mathcal{H}^{2} \\
\quad-\langle\bar{f}(t), \varphi\rangle_{W_{\varepsilon}{ }^{\prime}, W_{\varepsilon}}=-\int_{\Omega_{\varepsilon}} \pi_{\varepsilon}(t) \operatorname{div}\left(\partial_{t} \varphi\right) d x
\end{array}
$$

Integrating on $] 0, T\left[\right.$ and using that, by definition of $q_{\varepsilon}$,

$$
\int_{0}^{T} \int_{\Omega_{\varepsilon}} \pi_{\varepsilon}(t) \operatorname{div}\left(\partial_{t} \varphi\right) d x d t=\int_{0}^{T} \int_{\Omega_{\varepsilon}} q_{\varepsilon} \operatorname{div} \varphi d x d t
$$

we get the desired result and conclude the proof ot Proposition 3.2.

Unfolding method. In order to prove Theorem 3.3, we rely on an adaptation of the unfolding method (see e.g. $[4,17,18]$ ), which is strongly related to the two-scale convergence method $[2,38]$. This approach requires some extra notation.

For $k^{\prime} \in \mathbb{Z}^{2}$ and $\rho>0$, we introduce the sets

$$
C_{\rho}^{k^{\prime}}=\rho k^{\prime}+\rho Y^{\prime}, \quad Q_{\rho}^{k^{\prime}}=\Omega_{\varepsilon} \cap\left(C_{\rho}^{k^{\prime}} \times \mathbb{R}\right) .
$$

Remark 4.2. In the former definition, we have used the identification $\mathcal{T}^{2} \sim\left[0,1\left[{ }^{2}\right.\right.$ and considered $\Omega_{\varepsilon}$ as a subset of $\mathbb{R}^{3}$. This identification will be used tacitly in the rest of the paper.

Then, we define $\kappa: \mathbb{R}^{2} \rightarrow \mathbb{Z}^{2}$ by

$$
\kappa\left(x^{\prime}\right)=k^{\prime} \Leftrightarrow x^{\prime} \in C_{1}^{k^{\prime}} .
$$


Remark that $\kappa$ is well defined up to a set of measure zero in $\mathbb{R}^{2}$ (the set $\cup_{k^{\prime} \in \mathbb{Z}^{2}} \partial C_{1}^{k^{\prime}}$ ). Moreover, for every $\rho>0$ and $k^{\prime} \in \mathbb{Z}^{2}$, we have

$$
\kappa\left(\frac{x^{\prime}}{\rho}\right)=k^{\prime} \Leftrightarrow x^{\prime} \in C_{\rho}^{k^{\prime}} .
$$

Finally, for a.e. $x^{\prime} \in \mathbb{R}^{2}$ we define $C_{\varepsilon}\left(x^{\prime}\right)$ as the square $C_{\varepsilon}^{k^{\prime}}$ such that $x^{\prime}$ belongs to $C_{\varepsilon}^{k^{\prime}}$.

The first step in this approach is to introduce a microscopic variable $y$, as follows. Given $\left(w_{\varepsilon}, q_{\varepsilon}\right) \in$ $L^{3}(] 0, T\left[, W^{1,3}\left(\Omega_{\varepsilon}, \mathbb{R}^{3}\right)\right) \times L^{\frac{3}{2}}(] 0, T\left[, L_{0}^{\frac{3}{2}}\left(\Omega_{\varepsilon}, \mathbb{R}\right)\right)$, with $w_{\varepsilon}=0$ on $\Gamma^{0}$, a.e. in $] 0, T\left[\right.$, we define $\left(\widehat{w}_{\varepsilon}, \widehat{q}_{\varepsilon}\right)$ by

$$
\begin{aligned}
& \widehat{w}_{\varepsilon}\left(t, x^{\prime}, y\right)=w_{\varepsilon}\left(t, \varepsilon \kappa\left(\frac{x^{\prime}}{\varepsilon}\right)+\varepsilon y^{\prime}, \varepsilon y_{3}\right) \\
& \widehat{q}_{\varepsilon}\left(t, x^{\prime}, y\right)=q_{\varepsilon}\left(t, \varepsilon \kappa\left(\frac{x^{\prime}}{\varepsilon}\right)+\varepsilon y^{\prime}, \varepsilon y_{3}\right),
\end{aligned}
$$

for a.e. $\left.\left(t, x^{\prime}, y\right) \in\right] 0, T\left[\times \mathcal{T}^{2} \times \widehat{Y}_{\varepsilon}\right.$, with

$$
\widehat{Y}_{\varepsilon}=\left\{y \in Y^{\prime} \times \mathbb{R}:-\frac{a_{\varepsilon}}{\varepsilon} \Psi\left(y^{\prime}\right)<y_{3}<\frac{1}{\varepsilon}\right\} .
$$

Remark 4.3. For $k^{\prime} \in \mathbb{Z}^{2}$ the restriction of $\left(\widehat{w}_{\varepsilon}, \widehat{q}_{\varepsilon}\right)$ to $] 0, T\left[\times C_{\varepsilon}^{k^{\prime}} \times \widehat{Y}_{\varepsilon}\right.$ does not depend on $x^{\prime}$, while as a function of $y$ it is obtained from $\left(w_{\varepsilon}, q_{\varepsilon}\right)$ by using the change of variables

$$
y^{\prime}=\frac{x^{\prime}-\varepsilon k^{\prime}}{\varepsilon}, \quad y_{3}=\frac{x_{3}}{\varepsilon},
$$

which transforms $Q_{\varepsilon}^{k^{\prime}}$ into $\widehat{Y}_{\varepsilon}$.

In order to capture the asymptotic behaviour of $\left(w_{\varepsilon}, q_{\varepsilon}\right)$ near $] 0, T\left[\times \Gamma_{\varepsilon}\right.$, let us first study, in Lemmas 4.4 and 4.5 , the asymptotic behaviour of $\left(\widehat{w}_{\varepsilon}, \widehat{q}_{\varepsilon}\right)$ in $] 0, T\left[\times \mathcal{T}^{2} \times \widehat{Q}_{M}\right.$, for every $M>0$. These results represent a generalization to the evolutive case of Lemmas 4.5 and 5.6 in [43], originally stated for the stationary case in the particular case $p=3$.

Lemma 4.4. Let $\pi_{\varepsilon}$ be a bounded sequence in $L^{\infty}(] 0, T\left[, L^{3 / 2}\left(\Omega_{\varepsilon}, \mathbb{R}\right)\right)$. For a.e. $\left.\left(t, x^{\prime}, y\right) \in\right] 0, T\left[\times \mathcal{T}^{2} \times \widehat{Y}_{\varepsilon}\right.$ we define

$$
\widehat{\pi}_{\varepsilon}\left(t, x^{\prime}, y\right)=\pi_{\varepsilon}\left(t, \varepsilon \kappa\left(\frac{x^{\prime}}{\varepsilon}\right)+\varepsilon y^{\prime}, \varepsilon y_{3}\right) .
$$

Then there exists $\widehat{\pi} \in L^{\infty}(] 0, T\left[, L^{3 / 2}\left(\mathcal{T}^{2} \times \widehat{Q}, \mathbb{R}\right)\right)$ such that

$$
\forall M>0 \quad \varepsilon^{2 / 3} \widehat{\pi}_{\varepsilon} \rightarrow \widehat{\pi} \quad \text { weakly-* in } L^{\infty}(] 0, T\left[, L^{3 / 2}\left(\mathcal{T}^{2} \times \widehat{Q}_{M}, \mathbb{R}\right)\right) .
$$

Moreover, defining $q_{\varepsilon}=-\partial_{t} \pi_{\varepsilon}$ and $\widehat{q}=-\partial_{t} \widehat{\pi}$, there holds for every $\widehat{\varphi} \in C_{c}^{1}(] 0, T\left[\times \mathcal{T}^{2}, C_{\#}^{1}\left(\widehat{Q}, \mathbb{R}^{3}\right)\right)$ :

$$
\lim _{\varepsilon \rightarrow 0}\left[\varepsilon^{-1 / 3} \int_{(0, T) \times \Omega} q_{\varepsilon}(t, x)\left(\operatorname{div}_{\mathrm{y}} \widehat{\varphi}\right)\left(t, x^{\prime}, \frac{x}{\varepsilon}\right) d x d t\right]=\int_{(0, T) \times \mathcal{T}^{2} \times \widehat{Q}_{M}} \widehat{q} \operatorname{div}_{\mathrm{y}} \widehat{\varphi} d x^{\prime} d y d t .
$$


Proof. Let $M>0$ and $\varepsilon>0$. We write for a.e. $t \in] 0, T[$,

$$
\begin{aligned}
\varepsilon \int_{\mathcal{T}^{2} \times \widehat{Q}_{M}}\left|\widehat{\pi}_{\varepsilon}(t)\right|^{3 / 2} d x^{\prime} d y & =\varepsilon \int_{\mathcal{T}^{2} \times \widehat{Q}_{M}}\left|\pi_{\varepsilon}\left(t, \varepsilon \kappa\left(\frac{x^{\prime}}{\varepsilon}\right)+\varepsilon y^{\prime}, \varepsilon y_{3}\right)\right|^{3 / 2} d x^{\prime} d y \\
& =\varepsilon^{3} \sum_{k^{\prime} \in K_{\varepsilon}} \int_{\widehat{Q}_{M}}\left|\pi_{\varepsilon}\left(t, \varepsilon k^{\prime}+\varepsilon y^{\prime}, \varepsilon y_{3}\right)\right|^{3 / 2} d y \\
& =\sum_{k^{\prime} \in K_{\varepsilon}} \int_{C_{\varepsilon}^{k^{\prime}} \times(0, \varepsilon M)}\left|\pi_{\varepsilon}\left(t, x^{\prime}, x_{3}\right)\right|^{3 / 2} d x \\
& \leq \int_{\Omega_{\varepsilon}}\left|\pi_{\varepsilon}(t, x)\right|^{3 / 2} d x .
\end{aligned}
$$

Taking the essential supremum in the former inequality, we deduce that

$$
\left\|\varepsilon^{2 / 3} \widehat{\pi}_{\varepsilon}\right\|_{L^{\infty}(] 0, T\left[, L^{3 / 2}\left(\mathcal{T}^{2} \times \widehat{Q}_{M}, \mathbb{R}\right)\right)} \leq C .
$$

Since $L^{3}\left(\mathcal{T}^{2} \times \widehat{Q}_{M}, \mathbb{R}\right)$ is separable, the space $L^{1}(] 0, T\left[, L^{3}\left(\mathcal{T}^{2} \times \widehat{Q}_{M}, \mathbb{R}\right)\right)$ is separable. Hence, we obtain the convergence (4.17) by applying Banach Alaoglu theorem and a diagonal argument.

Finally, for any $\widehat{\varphi} \in C_{c}^{1}(] 0, T\left[\times \mathcal{T}^{2}, C_{\#}^{1}\left(\widehat{Q}, \mathbb{R}^{3}\right)\right)$, the limit (4.18) comes from the following computation:

$$
\begin{aligned}
& \lim _{\varepsilon \rightarrow 0}\left[\varepsilon^{-1 / 3} \int_{(0, T) \times \Omega_{\varepsilon}} q_{\varepsilon}(t, x)\left(\operatorname{div}_{\mathrm{y}} \widehat{\varphi}\right)\left(t, x^{\prime}, \frac{x}{\varepsilon}\right) d x d t\right] \\
& =\lim _{\varepsilon \rightarrow 0}\left[\varepsilon^{-1 / 3} \int_{(0, T) \times \Omega} \pi_{\varepsilon}(t, x)\left(\operatorname{div}_{\mathrm{y}}\left(\partial_{t} \widehat{\varphi}\right)\right)\left(t, x^{\prime}, \frac{x}{\varepsilon}\right) d x d t\right] \\
& =\lim _{\varepsilon \rightarrow 0}\left[\int_{(0, T) \times \mathcal{T}^{2} \times \widehat{Q}_{M}} \varepsilon^{2 / 3} \widehat{\pi}_{\varepsilon}\left(\operatorname{div}_{\mathrm{y}}\left(\partial_{t} \widehat{\varphi}\right)\right) d x^{\prime} d y d t\right] \\
& =\int_{(0, T) \times \mathcal{T}^{2} \times \widehat{Q}_{M}} \widehat{\pi} \operatorname{div}_{\mathrm{y}}\left(\partial_{t} \widehat{\varphi}\right) d x^{\prime} d y d t \\
& =\int_{(0, T) \times \mathcal{T}^{2} \times \widehat{Q}_{M}} \widehat{q} \operatorname{div}_{\mathrm{y}} \widehat{\varphi} d x^{\prime} d y d t .
\end{aligned}
$$

Lemma 4.5. We consider a sequence of functions $w_{\varepsilon} \in J_{\varepsilon}$, where $J_{\varepsilon}$ is defined by (2.22). Assume that there exists a constant $C>0$, such that

$$
\left\|w_{\varepsilon}\right\|_{H^{1}(] 0, T\left[, L^{2}\left(\Omega_{\varepsilon}, \mathbb{R}^{3}\right)\right)} \leq C, \quad\left\|w_{\varepsilon}\right\|_{L^{3}(] 0, T\left[, W^{1,3}\left(\Omega_{\varepsilon}, \mathbb{R}^{3}\right)\right)} \leq C .
$$

Assume that $w_{\varepsilon \mid \Omega}$ converges weakly to a function $w$ in $L^{3}(] 0, T\left[, W^{1,3}\left(\Omega, \mathbb{R}^{3}\right)\right.$ ) (this always holds for a subsequence). Then, the third component $w_{3}$ of $w$ vanishes on $] 0, T[\times \Gamma$.

Moreover, the following result holds.

(i) If $a_{\varepsilon} \approx \varepsilon^{\frac{5}{3}}$, with $a_{\varepsilon} / \varepsilon^{\frac{5}{3}} \rightarrow \lambda, 0<\lambda<+\infty$, then there exists $\widehat{w} \in L^{3}(] 0, T\left[, L^{3}\left(\mathcal{T}^{2}, \mathcal{V}^{3}\right)\right)$ with

$$
\left.\widehat{w}_{3}\left(t, x^{\prime}, y^{\prime}, 0\right)=-\lambda \nabla \Psi\left(y^{\prime}\right) \cdot w^{\prime}\left(t, x^{\prime}, 0\right), \quad \text { a.e. }\left(t, x^{\prime}, y^{\prime}\right) \in\right] 0, T\left[\times \mathcal{T}^{2} \times Y^{\prime},\right.
$$


such that for every $M>0$, the sequence $\widehat{w}_{\varepsilon}$ defined by (4.14) satisfies

$$
\varepsilon^{-\frac{2}{3}} D_{y} \widehat{w}_{\varepsilon} \rightarrow D_{y} \widehat{w} \text { in } L^{3}(] 0, T\left[\times \mathcal{T}^{2} \times \widehat{Q}_{M}, \mathbb{R}^{9}\right) .
$$

Besides, if $\operatorname{div} w_{\varepsilon}=0$ in $] 0, T\left[\times \Omega_{\varepsilon}\right.$, then

$$
\left.\operatorname{div}_{\mathrm{y}} \widehat{w}=0 \text { in }\right] 0, T\left[\times \mathcal{T}^{2} \times \widehat{Q} .\right.
$$

(ii) If $a_{\varepsilon} \gg \varepsilon^{\frac{5}{3}}$, then

$$
\left.w^{\prime}\left(t, x^{\prime}, 0\right) \cdot \nabla \Psi\left(y^{\prime}\right)=0 \text { a.e. }\left(t, x^{\prime}, y^{\prime}\right) \in\right] 0, T\left[\times \mathcal{T}^{2} \times Y^{\prime}\right.
$$

Proof. We divide the proof in five steps.

Step 1. Let us first prove that $w_{3}$ vanishes on $\Gamma$.

Since $w_{\varepsilon} \cdot \nu=0$ on $] 0, T\left[\times \Gamma_{\varepsilon}\right.$, for every $\varphi \in C_{c}^{1}(\Lambda, \mathbb{R})$ we have

$$
\int_{\Omega_{\varepsilon}} w_{\varepsilon} \nabla \varphi d x=-\int_{\Omega_{\varepsilon}}\left(\operatorname{div} w_{\varepsilon}\right) \varphi d x
$$

in $\mathcal{D}^{\prime}(0, T)$. Using

$$
\begin{gathered}
\left|\int_{\Omega_{\varepsilon} \backslash \Omega} w_{\varepsilon} \nabla \varphi d x\right| \leq\left(\int_{\Omega_{\varepsilon}}\left|w_{\varepsilon}\right|^{3} d x\right)^{\frac{1}{3}}\left(\int_{\Omega_{\varepsilon} \backslash \Omega}|\nabla \varphi|^{\frac{3}{2}} d x\right)^{\frac{2}{3}} \rightarrow 0, \\
\left|\int_{\Omega_{\varepsilon} \backslash \Omega}\left(\operatorname{div} w_{\varepsilon}\right) \varphi d x\right| \leq\left(\int_{\Omega_{\varepsilon}}\left|\operatorname{div} w_{\varepsilon}\right|^{3} d x\right)^{\frac{1}{3}}\left(\int_{\Omega_{\varepsilon} \backslash \Omega}|\varphi|^{\frac{3}{2}} d x\right)^{\frac{2}{3}} \rightarrow 0,
\end{gathered}
$$

and the weak convergence of $w_{\varepsilon}$ to $w$ in $L^{3}(] 0, T\left[, W^{1,3}\left(\Omega, \mathbb{R}^{3}\right)\right)$, we can pass to the limit in (4.23) to deduce

$$
\int_{\Omega} w \cdot \nabla \varphi d x=-\int_{\Omega}(\operatorname{div} w) \varphi d x
$$

in $\mathcal{D}^{\prime}(0, T)$, and then

$$
\int_{\Gamma} w_{3} \varphi d x^{\prime}=0, \quad \forall \varphi \in C_{c}^{1}(\Lambda, \mathbb{R})
$$

which proves $w_{3}=0$ on $] 0, T[\times \Gamma$.

Step 2. Let us obtain some estimates for the sequence $\widehat{w}_{\varepsilon}$ given by (4.14).

For $M>0$, the definition (4.14) of $\widehat{w}_{\varepsilon}$ proves for every $\varepsilon>0$ small enough

$$
\begin{aligned}
\int_{\mathcal{T}^{2} \times \widehat{Q}_{M}}\left|D_{y} \widehat{w}_{\varepsilon}\left(t, x^{\prime}, y\right)\right|^{3} d x^{\prime} d y & =\varepsilon^{5} \sum_{k^{\prime} \in K_{\varepsilon}} \int_{\widehat{Q}_{M}}\left|D w_{\varepsilon}\left(t, \varepsilon\left(k^{\prime}+y^{\prime}\right), \varepsilon y_{3}\right)\right|^{3} d y \\
& =\sum_{k^{\prime} \in K_{\varepsilon}} \varepsilon^{2} \int_{C_{\varepsilon}^{k^{\prime} \times(0, \varepsilon M)}}\left|D w_{\varepsilon}\right|^{3} d x \\
& \leq \varepsilon^{2} \int_{\Omega_{\varepsilon}}\left|D w_{\varepsilon}\right|^{3} d x
\end{aligned}
$$


Integrating between 0 and $T$, and taking into account (3.1)-(3.2), we have

$$
\int_{0}^{T} \int_{\mathcal{T}^{2} \times \widehat{Q}_{M}}\left|D_{y} \widehat{w}_{\varepsilon}\left(x^{\prime}, y\right)\right|^{3} d x^{\prime} d y d t \leq C \varepsilon^{2} .
$$

On the other hand, defining

$$
\bar{w}_{\varepsilon}\left(t, x^{\prime}\right)=\frac{1}{\varepsilon^{2}} \int_{C_{\varepsilon}\left(x^{\prime}\right)} w_{\varepsilon}\left(t, \tau^{\prime}, 0\right) d \tau^{\prime}=\int_{Y^{\prime}} \widehat{w}_{\varepsilon}\left(t, x^{\prime}, y^{\prime}, 0\right) d y^{\prime},
$$

and using the inequality

$$
\left.\int_{\widehat{Q}_{M}}\left|\widehat{w}_{\varepsilon}\left(t, x^{\prime}, y\right)-\bar{w}_{\varepsilon}\left(t, x^{\prime}\right)\right|^{3} d y \leq C_{M} \int_{\widehat{Q}_{M}}\left|D_{y} \widehat{w}_{\varepsilon}\right|^{3} d y, \quad \text { a.e. }\left(t, x^{\prime}\right) \in\right] 0, T\left[\times \mathcal{T}^{2},\right.
$$

where $C_{M}$ does not depend on $\varepsilon$ and integrating between 0 and $T$ we have

$$
\int_{0}^{T} \int_{\widehat{Q}_{M}}\left|\widehat{w}_{\varepsilon}\left(x^{\prime}, y\right)-\bar{w}_{\varepsilon}\left(x^{\prime}\right)\right|^{3} d y \leq C_{M} \int_{0}^{T} \int_{\widehat{Q}_{M}}\left|D_{y} \widehat{w}_{\varepsilon}\right|^{3} d y, \quad \text { a.e. } x^{\prime} \in \mathcal{T}^{2},
$$

Taking into account (4.25), we deduce that

$$
\left.\widehat{W}_{\varepsilon}=\frac{\widehat{w}_{\varepsilon}\left(t, x^{\prime}, y\right)-\bar{w}_{\varepsilon}\left(t, x^{\prime}\right)}{\varepsilon^{\frac{2}{3}}} \text { is bounded in } L^{3}(] 0, T\left[\times \mathcal{T}^{2}, W^{1,3}\left(\widehat{Q}_{M}, \mathbb{R}^{3}\right)\right)\right), \quad \forall M>0 .
$$

Thus, there exists $\widehat{w}:] 0, T\left[\times \mathcal{T}^{2} \times \widehat{Q} \rightarrow \mathbb{R}^{3}\right.$, such that, up to a subsequence,

$$
\widehat{W}_{\varepsilon} \rightarrow \widehat{w} \text { in } L^{3}(] 0, T\left[\times \mathcal{T}^{2}, W^{1,3}\left(\widehat{Q}_{M}, \mathbb{R}^{3}\right)\right), \quad \forall M>0,
$$

and then

$$
\varepsilon^{-\frac{2}{3}} D_{y} \widehat{w}_{\varepsilon} \rightarrow D_{y} \widehat{w} \text { in } L^{3}(] 0, T\left[\times \mathcal{T}^{2} \times Q_{M}, \mathbb{R}^{9}\right), \quad \forall M>0 .
$$

Passing to the limit by semicontinuity in inequalities (4.25) and (4.27) (this latest one after integration in $\mathcal{T}^{2}$ ), we get

$$
\int_{0}^{T} \int_{\mathcal{T}^{2} \times \widehat{Q}_{M}}\left|D_{y} \widehat{w}\right|^{3} d x^{\prime} d y d t \leq C, \quad \int_{0}^{T} \int_{\mathcal{T}^{2} \times \widehat{Q}_{M}}|\widehat{w}|^{3} d x^{\prime} d y d y \leq C_{M},
$$

and by the arbitrariness of $M$, once we prove the $Y^{\prime}$-periodicity of $\widehat{w}$ in $y^{\prime}$ (Step 3), then

$$
\widehat{w} \in L^{3}(] 0, T\left[\times \mathcal{T}^{2}, \mathcal{V}^{3}\right)
$$

Moreover, if we also assume that $\operatorname{div} w_{\varepsilon}=0$ in $] 0, T\left[\times \Omega_{\varepsilon}\right.$, then by definition (4.14) of $\widehat{w}_{\varepsilon}$, we have $\operatorname{div}_{y} \widehat{w}_{\varepsilon}=0$ in $] 0, T\left[\times \mathcal{T}^{2} \times \widehat{Q}_{M}\right.$, which together with (4.30) proves

$$
\left.\operatorname{div}_{y} \widehat{w}=0 \text { in }\right] 0, T\left[\times \mathcal{T}^{2} \times \widehat{Q} .\right.
$$

Step 3. Let us prove that $\widehat{w}$ is $Y^{\prime}$-periodic in $y^{\prime}$.

We observe that by definition (4.14) of $\widehat{w}_{\varepsilon}$, for every $M>0$, we have

$$
\left.\widehat{w}_{\varepsilon}\left(t, x_{1}+\varepsilon, x_{2},-\frac{1}{2}, y_{2}, y_{3}\right)=\widehat{w}_{\varepsilon}\left(t, x^{\prime}, \frac{1}{2}, y_{2}, y_{3}\right), \text { a.e. }\left(t, x^{\prime}, y_{2}, y_{3}\right) \in\right] 0, T\left[\times \mathcal{T}^{2} \times\left(-\frac{1}{2}, \frac{1}{2}\right) \times(0, M) .\right.
$$


Therefore the sequence $\widehat{W}_{\varepsilon}$ satisfies

$$
\begin{array}{r}
\widehat{W}_{\varepsilon}\left(t, x_{1}+\varepsilon, x_{2},-\frac{1}{2}, y_{2}, y_{3}\right)-\widehat{W}_{\varepsilon}\left(t, x^{\prime}, \frac{1}{2}, y_{2}, y_{3}\right)=\frac{-\bar{w}_{\varepsilon}\left(t, x_{1}+\varepsilon, x_{2}\right)+\bar{w}_{\varepsilon}\left(t, x^{\prime}\right)}{\varepsilon^{\frac{2}{3}}}, \\
\text { a.e. } \left.\left(t, x^{\prime}, y_{2}, y_{3}\right) \in\right] 0, T\left[\times \mathcal{T}^{2} \times\left(-\frac{1}{2}, \frac{1}{2}\right) \times(0, M) .\right.
\end{array}
$$

where $\bar{w}_{\varepsilon}$ is defined by $(4.26)$. Using the fact that $w_{\varepsilon}(t, \cdot)$ is bounded in $L^{3}(\Gamma)^{3}$, we can proceed as the proof of Lemma 4.6 in [43] to deduce that the right-hand side of this equality tends to zero in the sense of distributions in $\mathcal{T}^{2}$. Therefore, passing to the limit in (4.33) by (4.29), and taking into account the arbitrariness of $M$ we get

$$
\left.\widehat{w}\left(t, x^{\prime},-\frac{1}{2}, y_{2}, y_{3}\right)-\widehat{w}\left(t, x^{\prime}, \frac{1}{2}, y_{2}, y_{3}\right)=0 \text { a.e. }\left(t, x^{\prime}, y_{2}, y_{3}\right) \in\right] 0, T\left[\times \mathcal{T}^{2} \times\left(-\frac{1}{2}, \frac{1}{2}\right) \times \mathbb{R} .\right.
$$

Analogously, we can prove

$$
\left.\widehat{w}\left(t, x^{\prime}, y_{1},-\frac{1}{2}, y_{3}\right)-\widehat{w}\left(t, x^{\prime}, y_{1}, \frac{1}{2}, y_{3}\right)=0 \text { a.e. }\left(t, x^{\prime}, y_{1}, y_{3}\right) \in\right] 0, T\left[\times \mathcal{T}^{2} \times\left(-\frac{1}{2}, \frac{1}{2}\right) \times \mathbb{R} .\right.
$$

These equalities prove that $\widehat{w}$ is periodic with respect to $Y^{\prime}$.

Step 4. Since $w_{\varepsilon}$ and $\partial_{t} w_{\varepsilon}$ are uniformly bounded in $L^{3}(] 0, T\left[, W^{1,3}\left(\Omega, \mathbb{R}^{3}\right)\right)$ and $L^{2}(] 0, T\left[\times \Gamma, \mathbb{R}^{3}\right)$ respectively, by compactness of the embedding of $W^{1,3}(\Omega)$ into $L^{2}(\Gamma)$, one can apply Aubin-Lions theorem to deduce that $w_{\varepsilon}$ is precompact in $L^{3}(] 0, T\left[, L^{2}\left(\Gamma, \mathbb{R}^{3}\right)\right)$. Up to a subsequence, this implies the strong convergence of $\bar{w}_{\varepsilon}$ to $w\left(t, x^{\prime}, 0\right)$ in $L^{3}(] 0, T\left[, L^{2}\left(\Gamma, \mathbb{R}^{3}\right)\right)$. Thus, by (4.28), we deduce

$$
\widehat{w}_{\varepsilon}\left(t, x^{\prime}, y\right) \rightarrow w\left(t, x^{\prime}, 0\right) \quad \text { in } L^{3}(] 0, T\left[\times \mathcal{T}^{2}, W^{1,3}\left(\widehat{Q}_{M}, \mathbb{R}^{3}\right)\right), \quad \forall M>0 .
$$

Step 5. Using the change of variables (4.16), which defines $\widehat{w}_{\varepsilon}$, in the equality $w_{\varepsilon} \nu=0$ on $\Gamma_{\varepsilon}$, we get

$$
\frac{a_{\varepsilon}}{\varepsilon} \nabla \Psi\left(y^{\prime}\right) \cdot \widehat{w}_{\varepsilon}^{\prime}\left(t, x^{\prime}, y^{\prime},-\frac{a_{\varepsilon}}{\varepsilon} \Psi\left(y^{\prime}\right)\right)+\widehat{w}_{\varepsilon, 3}\left(t, x^{\prime}, y^{\prime},-\frac{a_{\varepsilon}}{\varepsilon} \Psi\left(y^{\prime}\right)\right)=0,
$$

a.e. in $] 0, T\left[\times \mathcal{T}^{2} \times Y^{\prime}\right.$. Thanks to (4.35) and (4.25), we have then

$$
\begin{aligned}
& \left|\frac{a_{\varepsilon}}{\varepsilon} \nabla \Psi\left(y^{\prime}\right) \cdot \widehat{w}_{\varepsilon}^{\prime}\left(t, x^{\prime}, y^{\prime}, 0\right)+\widehat{w}_{\varepsilon, 3}\left(t, x^{\prime}, y^{\prime}, 0\right)\right| \\
& \leq \int_{-\frac{a_{\varepsilon}}{\varepsilon} \Psi\left(y^{\prime}\right)}^{0}\left|\frac{a_{\varepsilon}}{\varepsilon} \nabla \Psi\left(y^{\prime}\right) \cdot \partial_{y_{3}} \widehat{w}_{\varepsilon}^{\prime}\left(t, x^{\prime}, y^{\prime}, s\right)+\partial_{y_{3}} \widehat{w}_{\varepsilon, 3}\left(t, x^{\prime}, y^{\prime}, s\right)\right| d s \\
& \left.\leq C\left(\frac{a_{\varepsilon}}{\varepsilon}\right)^{\frac{2}{3}}\left(\int_{-\frac{a_{\varepsilon}}{\varepsilon} \Psi\left(y^{\prime}\right)}^{0}\left|\partial_{y_{3}} \widehat{w}_{\varepsilon}\left(t, x^{\prime}, y^{\prime}, s\right)\right|^{3} d s\right)^{\frac{1}{3}} \text { a.e. }\left(t, x^{\prime}, y^{\prime}\right) \in\right] 0, T\left[\times \mathcal{T}^{2} \times Y^{\prime} .\right.
\end{aligned}
$$

Taking the power 3 , integrating in $\mathcal{T}^{2} \times Y^{\prime}$ and using (4.25) we then deduce

$$
\int_{\mathcal{T}^{2} \times Y^{\prime}}\left|\frac{a_{\varepsilon}}{\varepsilon} \nabla \Psi\left(y^{\prime}\right) \cdot \widehat{w}_{\varepsilon}^{\prime}\left(t, x^{\prime}, y^{\prime}, 0\right)+\widehat{w}_{\varepsilon, 3}\left(t, x^{\prime}, y^{\prime}, 0\right)\right|^{3} d x^{\prime} d y^{\prime} \leq C a_{\varepsilon}^{2},
$$


a.e. in $] 0, T[$, which implies

$$
\begin{aligned}
& \int_{\mathcal{T}^{2} \times Y^{\prime}} \mid \frac{a_{\varepsilon}}{\varepsilon} \nabla \Psi\left(y^{\prime}\right) \cdot \widehat{w}_{\varepsilon}^{\prime}\left(t, x^{\prime}, y^{\prime}, 0\right)+\widehat{w}_{\varepsilon, 3}\left(t, x^{\prime}, y^{\prime}, 0\right) \\
&-\left.\int_{Y^{\prime}}\left(\frac{a_{\varepsilon}}{\varepsilon} \nabla \Psi\left(z^{\prime}\right) \cdot \widehat{w}_{\varepsilon}^{\prime}\left(t, x^{\prime}, z^{\prime}, 0\right)+\widehat{w}_{\varepsilon, 3}\left(t, x^{\prime}, z^{\prime}, 0\right)\right) d z^{\prime}\right|^{3} d x^{\prime} d y^{\prime} \leq C a_{\varepsilon}^{2},
\end{aligned}
$$

a.e. in $] 0, T$. Integrating between 0 and $T$ and dividing by $\varepsilon^{2}$, and taking into account that $\nabla \Psi$ has mean value zero in $Y^{\prime}$, we get

$$
\begin{gathered}
\int_{0}^{T} \int_{\mathcal{T}^{2} \times Y^{\prime}} \mid \frac{a_{\varepsilon}}{\varepsilon^{\frac{5}{3}}} \nabla \Psi\left(y^{\prime}\right) \cdot \widehat{w}_{\varepsilon}^{\prime}\left(t, x^{\prime}, y^{\prime}, 0\right)-\frac{a_{\varepsilon}}{\varepsilon} \int_{Y^{\prime}} \nabla \Psi\left(z^{\prime}\right) \cdot\left(\frac{\widehat{w}_{\varepsilon}^{\prime}\left(t, x^{\prime}, z^{\prime}, 0\right)-\bar{w}_{\varepsilon}^{\prime}\left(t, x^{\prime}\right)}{\varepsilon^{\frac{2}{3}}}\right) d z^{\prime} \\
+\left.\frac{\widehat{w}_{\varepsilon, 3}\left(t, x^{\prime}, y^{\prime}, 0\right)-\bar{w}_{\varepsilon, 3}\left(t, x^{\prime}\right)}{\varepsilon^{\frac{2}{3}}}\right|^{3} d x^{\prime} d y^{\prime} d t \leq C\left(\frac{a_{\varepsilon}}{\varepsilon}\right)^{2} \rightarrow 0 .
\end{gathered}
$$

Thanks to (2.1) and taking into account the definition (3.5) of $\lambda$, we deduce:

- If $\lambda=+\infty$, statement (4.36) shows that

$$
\frac{a_{\varepsilon}}{\varepsilon^{\frac{5}{3}}} \nabla \Psi\left(y^{\prime}\right) \cdot \widehat{w}_{\varepsilon}^{\prime}\left(t, x^{\prime}, y^{\prime}, 0\right) \text { is bounded in } L^{3}(] 0, T\left[\times \mathcal{T}^{2} \times Y^{\prime}, \mathbb{R}\right),
$$

and then that $\nabla \Psi\left(y^{\prime}\right) \cdot \widehat{w}_{\varepsilon}^{\prime}\left(t, x^{\prime}, y^{\prime}, 0\right)$ tends to zero in $L^{3}(] 0, T\left[\times \mathcal{T}^{2} \times Y^{\prime}, \mathbb{R}\right)$. By (4.34), this proves (4.22).

- If $\lambda \in(0,+\infty)$, proceeding as in Step 4 we can pass to the limit in (4.36) to deduce (4.19).

Proof of Theorem 3.3. First of all, thanks to (3.2), convergences (3.6) hold.

The proof will be carried out by using suitable test functions $\varphi_{\varepsilon}$ in the variational formulation (3.4). We will divide the proof in several steps.

Step 1. Obtaining the limit system. We consider $\phi \in C_{c}^{1}([0, T], \mathbb{R}), \varphi \in C_{c}^{1}(\Lambda, \mathbb{R})^{3}, \varphi_{3}\left(x^{\prime}, 0\right)=0$, $\widehat{\varphi} \in C_{c}^{1}\left(\mathcal{T}^{2}, C_{\#}^{1}\left(\widehat{Q}, \mathbb{R}^{3}\right)\right)$, with $D_{y} \widehat{\varphi}\left(x^{\prime}, y\right)=0$ a.e. in $\left\{y_{3}>M\right\}$, for some $M>0$, such that

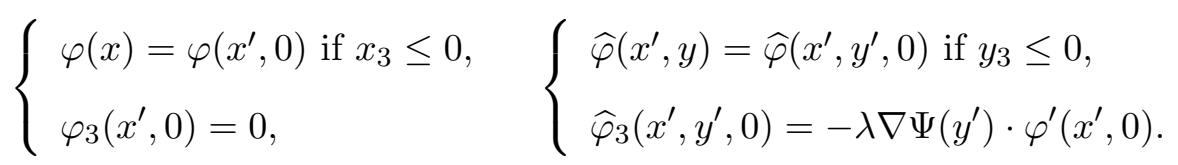

Besides, we take $\zeta \in C^{\infty}(\mathbb{R})$ such that

$$
\zeta\left(x_{3}\right)=\left\{\begin{array}{c}
1 \text { if } x_{3}<\frac{1}{3} \\
0 \text { if } x_{3}>\frac{2}{3}
\end{array}\right.
$$

and $R_{\varepsilon}>0$ such that

$$
R_{\varepsilon} \rightarrow \infty, \quad R_{\varepsilon}\left(\left|\frac{a_{\varepsilon}}{\varepsilon^{\frac{5}{3}}}-\lambda\right|^{3}+\left(\frac{a_{\varepsilon}}{\varepsilon}\right)^{3}\right) \rightarrow 0
$$


Then, we define $\varphi_{\varepsilon} \in W^{1,3}\left(\Omega_{\varepsilon}\right)^{3}$ by

$$
\left\{\begin{array}{l}
\varphi_{\varepsilon}^{\prime}(x)=\varphi^{\prime}(x)+\varepsilon^{\frac{2}{3}} \widehat{\varphi}^{\prime}\left(x^{\prime}, \frac{x}{\varepsilon}\right) \zeta\left(x_{3}\right) \\
\varphi_{\varepsilon, 3}(x)=\varphi_{3}(x)+\varepsilon^{\frac{2}{3}}\left[\widehat{\varphi}_{3}\left(x^{\prime}, \frac{x}{\varepsilon}\right) \zeta\left(x_{3}\right)+\right. \\
\left.\quad+\zeta\left(\frac{x_{3}}{\varepsilon R_{\varepsilon}}\right) \nabla \Psi\left(\frac{x^{\prime}}{\varepsilon}\right) \cdot\left(\left(\lambda-\frac{a_{\varepsilon}}{\varepsilon^{\frac{5}{3}}}\right) \varphi^{\prime}\left(x^{\prime}, 0\right)-\frac{a_{\varepsilon}}{\varepsilon} \widehat{\varphi}^{\prime}\left(x^{\prime}, \frac{x^{\prime}}{\varepsilon}, 0\right)\right)\right] .
\end{array}\right.
$$

Thanks to $\varphi(x)$ and $\widehat{\varphi}\left(x^{\prime}, y\right)$ equal zero for $x^{\prime}$ outside a compact subset of $\mathcal{T}^{2}$ and (4.37), the sequence $v_{\varepsilon}(t, x)=\phi(t) \varphi_{\varepsilon}(x)$ satisfies

$$
\left.v_{\varepsilon}=0 \text { on }\right] 0, T\left[\times T_{0}, \quad v_{\varepsilon} \cdot \nu=0 \text { on }\right] 0, T\left[\times \Gamma_{\varepsilon} .\right.
$$

Thus, we can take such $v_{\varepsilon}$ as test function in (3.4). The problem is to pass to the limit in the different terms which appears in (3.4). Before, we remark that since $D_{y} \widehat{\varphi}=0$ a.e. in $\left\{y_{3}>M\right\}$ and that $\zeta=1$ a.e. on $\left\{x_{3}<1 / 3\right\}$, we have

$$
D \varphi_{\varepsilon}(x)=D \varphi(x)+\varepsilon^{-\frac{1}{3}} D_{y} \widehat{\varphi}\left(x^{\prime}, \frac{x}{\varepsilon}\right)+h_{\varepsilon}(x),
$$

where, using that $\varphi, \widehat{\varphi}$ and $\zeta$ are bounded and have bounded derivatives, the function $h_{\varepsilon} \in C^{0}([0, T] \times$ $\left.\bar{\Omega}_{\varepsilon}, \mathbb{R}^{9}\right)$ satisfies

$$
\left|h_{\varepsilon}\right| \leq C \varepsilon^{\frac{2}{3}}+C\left[\left(\frac{1}{\varepsilon^{\frac{1}{3}} R_{\varepsilon}}+\frac{1}{\varepsilon^{\frac{1}{3}}}\right)\left(\left|\frac{a_{\varepsilon}}{\varepsilon^{\frac{5}{3}}}-\lambda\right|+\frac{a_{\varepsilon}}{\varepsilon}\right)+\varepsilon^{\frac{2}{3}}\left|\frac{a_{\varepsilon}}{\varepsilon^{\frac{5}{3}}}-\lambda\right|\right] \chi_{\left\{x_{3}<\frac{2}{3} \varepsilon R_{\varepsilon}\right\}},
$$

a.e. in $\Omega_{\varepsilon}$. Using that $R_{\varepsilon}$ tends to infinity, we get

$$
\left|h_{\varepsilon}\right| \leq C \varepsilon^{\frac{2}{3}}+C\left[\frac{1}{\varepsilon^{\frac{1}{3}}}\left|\frac{a_{\varepsilon}}{\varepsilon^{\frac{5}{3}}}-\lambda\right|+\frac{a_{\varepsilon}}{\varepsilon^{1+\frac{1}{3}}}\right] \chi_{\left\{x_{3}<\frac{2}{3} \varepsilon R_{\varepsilon}\right\}}, \text { a.e. in } \Omega_{\varepsilon} .
$$

Therefore, by (4.39), we have

$$
\int_{\Omega_{\varepsilon}}\left|h_{\varepsilon}\right|^{3} d x \leq O_{\varepsilon}+C R_{\varepsilon}\left(\left|\frac{a_{\varepsilon}}{\varepsilon^{\frac{5}{3}}}-\lambda\right|^{3}+\left(\frac{a_{\varepsilon}}{\varepsilon}\right)^{3}\right)=O_{\varepsilon} .
$$

Taking $v_{\varepsilon}$ as test function in (3.4) and using that $\left\|w_{\varepsilon}\right\|_{L^{3}(] 0, T\left[, W^{1,3}\left(\Omega_{\varepsilon}, \mathbb{R}^{3}\right)\right)}$ and $\left\|q_{\varepsilon}\right\|_{L^{\infty}(] 0, T\left[, L^{\frac{3}{2}}\left(\Omega_{\varepsilon}, \mathbb{R}\right)\right)}$ are bounded, $\left\|\varphi_{\varepsilon}-\varphi\right\|_{C^{0}\left(\bar{\Omega}_{\varepsilon}, \mathbb{R}\right)^{3}}$ tends to zero, (4.40) and (4.41), we get

$$
\begin{aligned}
& \int_{0}^{T} \phi(t) \int_{\Omega_{\varepsilon}} \partial_{t} w_{\varepsilon}(t) \cdot \varphi d x d t+\int_{0}^{T} \int_{\Omega_{\varepsilon}}\left(w_{\varepsilon}(t) \cdot \nabla\right) w_{\varepsilon}(t) \varphi d x d t \\
& +\int_{0}^{T} \phi(t) \int_{\Omega_{\varepsilon}} S\left(D w_{\varepsilon}(t)\right):\left(D \varphi+\varepsilon^{-\frac{1}{3}} D_{y} \widehat{\varphi}\left(x^{\prime}, \frac{x}{\varepsilon}\right)\right) d x d t \\
& -\int_{0}^{T} \phi(t) \int_{\Omega_{\varepsilon}} q_{\varepsilon}\left(\operatorname{div} \varphi+\varepsilon^{-\frac{1}{3}} \operatorname{div}_{\mathrm{y}} \widehat{\varphi}\left(x^{\prime}, \frac{x}{\varepsilon}\right)\right) d x d t+\beta \int_{0}^{T} \phi(t) \int_{\Gamma_{\varepsilon}} w_{\varepsilon} \cdot \varphi d \mathcal{H}^{2} d t \\
& =\int_{0}^{T} \phi(t) \int_{\Omega_{\varepsilon}} \bar{f} \cdot \varphi d x d t+O_{\varepsilon} .
\end{aligned}
$$


Let us now pass to the limit in every term of the above equality.

- First term of (4.42). Since $\left|\Omega_{\varepsilon} \backslash \Omega\right| \rightarrow 0$ and $\partial_{t} w_{\varepsilon} \in L^{\infty}(] 0, T\left[, L^{2}\left(\Omega_{\varepsilon}, \mathbb{R}^{3}\right)\right)$, then we have

$$
\int_{\Omega_{\varepsilon}} \partial_{t} w_{\varepsilon} \cdot \varphi d x=\int_{\Omega} \partial_{t} w_{\varepsilon} \cdot \varphi d x+O_{\varepsilon}
$$

Moreover, we have

$$
\int_{\Omega_{\varepsilon}} \partial_{t} w_{\varepsilon}(t) \cdot \varphi d x=\frac{d}{d t}\left(\int_{\Omega_{\varepsilon}} w_{\varepsilon}(t) \cdot v d x\right)
$$

and so

$$
\frac{d}{d t}\left(\int_{\Omega_{\varepsilon}} w_{\varepsilon}(t) \cdot \varphi d x\right) \phi(t)=\frac{d}{d t}\left(\phi(t) \int_{\Omega_{\varepsilon}} w_{\varepsilon}(t) \cdot v d x\right)-\partial_{t} \phi(t) \int_{\Omega_{\varepsilon}} w_{\varepsilon}(t) \cdot v d x
$$

Thus, we get

$$
\int_{0}^{T} \phi(t) \int_{\Omega_{\varepsilon}} \partial_{t} w_{\varepsilon}(t) \cdot v d x d t=-\int_{0}^{T} \partial_{t} \phi(t) \int_{\Omega_{\varepsilon}} w_{\varepsilon}(t) \cdot \varphi d x d t
$$

Since $w_{\varepsilon}$ converges weakly-* to $w$ in $L^{\infty}(] 0, T\left[, L^{2}\left(\Omega, \mathbb{R}^{3}\right)\right)$ and taking $\varphi \partial_{t} \phi(t) \in L^{1}(] 0, T\left[, L^{2}\left(\Lambda, \mathbb{R}^{3}\right)\right)$, we have

$$
\begin{aligned}
& \int_{0}^{T} \phi(t) \int_{\Omega_{\varepsilon}} \partial_{t} w_{\varepsilon}(t) \cdot v d x d t \\
& \quad=-\phi(0) \int_{\Omega_{\varepsilon}} w^{0} \cdot \varphi d x-\int_{0}^{T} \partial_{t} \phi(t) \int_{\Omega_{\varepsilon}} w(t) \cdot \varphi d x d t+O_{\varepsilon} \\
& \quad=\int_{0}^{T} \phi(t) \int_{\Omega_{\varepsilon}} \partial_{t} w(t) \cdot v d x d t+O_{\varepsilon} .
\end{aligned}
$$

- Second term of (4.42). From $\operatorname{div} w_{\varepsilon}=0$ and $w_{\varepsilon} \nu=0$ on $\Gamma_{\varepsilon}$, we have

$$
\int_{0}^{T} \int_{\Omega_{\varepsilon}}\left(w_{\varepsilon}(t) \cdot \nabla\right) w_{\varepsilon}(t) \phi(t) \varphi d x=-\int_{0}^{T} \phi(t) \int_{\Omega_{\varepsilon}}\left(w_{\varepsilon}(t) \cdot \nabla \varphi\right) w_{\varepsilon} d x d t
$$

Taking into account that $\left|\Omega_{\varepsilon} \backslash \Omega\right| \rightarrow 0$, and $w_{\varepsilon} \in J_{\varepsilon}$, we have

$$
\int_{0}^{T} \int_{\Omega_{\varepsilon}}\left(w_{\varepsilon}(t) \cdot \nabla\right) w_{\varepsilon}(t) \phi(t) \varphi d x=-\int_{0}^{T} \phi(t) \int_{\Omega}\left(w_{\varepsilon}(t) \cdot \nabla \varphi\right) w_{\varepsilon}(t) d x d t+O_{\varepsilon}
$$

Since $w_{\varepsilon} \in L^{3}(] 0, T\left[, W^{1,3}\left(\Omega, \mathbb{R}^{3}\right)\right)$ and $\partial_{t} w_{\varepsilon} \in L^{2}(] 0, T\left[\times \Omega, \mathbb{R}^{3}\right)$, by applying Aubin-Lions theorem we deduce that $w_{\varepsilon}$ is compact in $L^{3}(] 0, T\left[, L^{3}\left(\Omega, \mathbb{R}^{3}\right)\right)$ wich implies the strong convergence of $w_{\varepsilon}$ to $w$ in $L^{3}(] 0, T\left[, L^{3}\left(\Omega, \mathbb{R}^{3}\right)\right)$. This together with weak convergence of $w_{\varepsilon}$ to $w$ in $L^{3}(] 0, T\left[, W^{1,3}\left(\Omega, \mathbb{R}^{3}\right)\right)$ implies

$$
\int_{0}^{T} \int_{\Omega_{\varepsilon}}\left(w_{\varepsilon}(t) \cdot \nabla\right) w_{\varepsilon}(t) \phi(t) \varphi d x=-\int_{0}^{T} \phi(t) \int_{\Omega}(w(t) \cdot \nabla \varphi) w(t) d x d t+O_{\varepsilon} .
$$

- Third term of (4.42). Taking into account that

$$
\int_{\Omega_{\varepsilon} \backslash \Omega}\left|D \varphi+\varepsilon^{-\frac{1}{3}} D_{y} \widehat{\varphi}\left(x^{\prime}, \frac{x}{\varepsilon}\right)\right|^{3} d x \leq \frac{C}{\varepsilon}\left|\Omega_{\varepsilon} \backslash \Omega\right| \leq C \frac{a_{\varepsilon}}{\varepsilon}=O_{\varepsilon},
$$


we divide the term in two integrals

$$
\begin{aligned}
& \int_{0}^{T} \phi(t) \int_{\Omega_{\varepsilon}} S\left(D w_{\varepsilon}(t)\right):\left(D \varphi+\varepsilon^{-\frac{1}{3}} D_{y} \widehat{\varphi}\left(x^{\prime}, \frac{x}{\varepsilon}\right)\right) d x d t \\
& =\int_{0}^{T} \phi(t) \int_{\Omega} S\left(D w_{\varepsilon}(t)\right): D \varphi d x d t+\varepsilon^{-\frac{1}{3}} \int_{0}^{T} \phi(t) \int_{\Omega} S\left(D w_{\varepsilon}(t)\right): D_{y} \widehat{\varphi}\left(x^{\prime}, \frac{x}{\varepsilon}\right) d x d t+O_{\varepsilon} .
\end{aligned}
$$

Since $w_{\varepsilon} \in L^{3}(] 0, T\left[, W^{1,3}\left(\Omega_{\varepsilon}\right)^{3}\right)$, then $S\left(D w_{\varepsilon}\right)$ is bounded in $L^{\frac{3}{2}}(] 0, T\left[, L^{\frac{3}{2}}\left(\Omega_{\varepsilon}, \mathbb{R}^{9}\right)\right)$, and so there exists $\xi \in L^{\frac{3}{2}}(] 0, T\left[, L^{\frac{3}{2}}\left(\Omega, \mathbb{R}^{9}\right)\right)$ such that

$$
S\left(D w_{\varepsilon}\right) \rightarrow \xi \text { in } L^{\frac{3}{2}}(] 0, T\left[, L^{\frac{3}{2}}\left(\Omega, \mathbb{R}^{9}\right)\right) .
$$

Then the first term in the right-hand side of (4.46) reads

$$
\int_{0}^{T} \phi(t) \int_{\Omega} S\left(D w_{\varepsilon}\right): D \varphi d x d t=\int_{0}^{T} \phi(t) \int_{\Omega} \xi: D \varphi d x d t+O_{\varepsilon} .
$$

In the second term of the right-hand side of (4.46), we introduce the sequence $\widehat{w}_{\varepsilon}$ defined by (4.14). By $w_{\varepsilon} \in L^{3}(] 0, T\left[, W^{1,3}\left(\Omega_{\varepsilon}, \mathbb{R}^{3}\right)\right)$ and Lemma 4.5 , we have that $\widehat{S}\left(\varepsilon^{-\frac{2}{3}} D_{y} \widehat{w}_{\varepsilon}\right)$ is bounded in $L^{\frac{3}{2}}(] 0, T\left[, L^{\frac{3}{2}}\left(\mathcal{T}^{2} \times\right.\right.$ $\left.\left.\widehat{Q}_{M}, \mathbb{R}^{9}\right)\right) \forall M>0$, and so there exists $\widehat{\xi} \in L^{\frac{3}{2}}(] 0, T\left[, L^{\frac{3}{2}}\left(\mathcal{T}^{2} \times \widehat{Q}, \mathbb{R}^{9}\right)\right)$ such that

$$
\widehat{S}\left(\varepsilon^{-\frac{2}{3}} D_{y} \widehat{w}_{\varepsilon}\right) \rightarrow \widehat{\xi} \text { in } L^{\frac{3}{2}}(] 0, T\left[, L^{\frac{3}{2}}\left(\mathcal{T}^{2} \times \widehat{Q}, \mathbb{R}^{9}\right)\right) .
$$

Moreover, we have

$$
\begin{aligned}
& \varepsilon^{-\frac{1}{3}} \int_{0}^{T} \phi(t) \int_{\Omega} S\left(D w_{\varepsilon}\right): D_{y} \widehat{\varphi}\left(x^{\prime}, \frac{x}{\varepsilon}\right) d x d t \\
& =\frac{\varepsilon^{-\frac{1}{3}}}{R e} \int_{0}^{T} \phi(t) \int_{\Omega} D w_{\varepsilon}: D_{y} \widehat{\varphi}\left(x^{\prime}, \frac{x}{\varepsilon}\right) d x d t+\varepsilon^{-\frac{1}{3}} \int_{0}^{T} \phi(t) \int_{\Omega} \widehat{S}\left(D w_{\varepsilon}\right): D_{y} \widehat{\varphi}\left(x^{\prime}, \frac{x}{\varepsilon}\right) d x d t \\
& =\frac{\varepsilon^{-\frac{1}{3}}}{R e} \int_{\mathcal{T}^{2}} \int_{0}^{T} \phi(t) \int_{\widehat{Q}_{M}} D_{y} \widehat{w}_{\varepsilon}: D_{y} \widehat{\varphi} d y d x^{\prime} d t+\varepsilon^{\frac{2}{3}} \int_{0}^{T} \phi(t) \int_{\mathcal{T}^{2}} \int_{\widehat{Q}_{M}} \widehat{S}\left(\varepsilon^{-1} D_{y} \widehat{w}_{\varepsilon}\right): D_{y} \widehat{\varphi} d y d x^{\prime} d t+O_{\varepsilon} \\
& =\frac{\varepsilon^{\frac{1}{3}}}{R e} \int_{0}^{T} \phi(t) \int_{\mathcal{T}^{2}} \int_{\widehat{Q}_{M}} \varepsilon^{-\frac{2}{3}} D_{y} \widehat{w}_{\varepsilon}: D_{y} \widehat{\varphi} d y d x^{\prime} d t+\int_{0}^{T} \phi(t) \int_{\mathcal{T}^{2}} \int_{\widehat{Q}_{M}} \widehat{S}\left(\varepsilon^{-\frac{2}{3}} D_{y} \widehat{w}_{\varepsilon}\right): D_{y} \widehat{\varphi} d y d x^{\prime} d t+O_{\varepsilon} \\
& =\int_{0}^{T} \phi(t) \int_{\mathcal{T}^{2}} \int_{\widehat{Q}_{M}} \widehat{S}\left(\varepsilon^{-\frac{2}{3}} D_{y} \widehat{w}_{\varepsilon}\right): D_{y} \widehat{\varphi} d y d x^{\prime} d t+O_{\varepsilon} \\
& =\int_{0}^{T} \phi(t) \int_{\mathcal{T}^{2} \times \widehat{Q}} \widehat{\xi}: D_{y} \widehat{\varphi} d x^{\prime} d y d t+O_{\varepsilon} .
\end{aligned}
$$

Thus, (4.48) and the above equality imply

$$
\begin{aligned}
\int_{0}^{T} \phi(t) & \int_{\Omega_{\varepsilon}} S\left(D w_{\varepsilon}\right):\left(D \varphi+\varepsilon^{-\frac{1}{3}} D_{y} \widehat{\varphi}\left(x^{\prime}, \frac{x}{\varepsilon}\right)\right) d x d t \\
& =\int_{0}^{T} \phi(t) \int_{\Omega} \xi: D \varphi d x d t+\int_{0}^{T} \phi(t) \int_{\mathcal{T}^{2} \times \widehat{Q}} \widehat{\xi}: D_{y} \widehat{\varphi} d x^{\prime} d y d t+O_{\varepsilon} .
\end{aligned}
$$


- Fourth term of (4.42). Similarly to the previous term, taking into account (4.45), we split this term as follows

$$
\begin{aligned}
& \int_{0}^{T} \int_{\Omega_{\varepsilon}} q_{\varepsilon}(t)\left(\operatorname{div} \varphi+\varepsilon^{-\frac{1}{3}} \operatorname{div}_{\mathrm{y}} \widehat{\varphi}\left(x^{\prime}, \frac{x}{\varepsilon}\right)\right) d x d t \\
& =\int_{0}^{T} \int_{\Omega} q_{\varepsilon}(t) \operatorname{div} \varphi d x d t+\varepsilon^{-\frac{1}{3}} \int_{0}^{T} \int_{\Omega} q_{\varepsilon}(t) \operatorname{div}_{\mathrm{y}} \widehat{\varphi}\left(x^{\prime}, \frac{x}{\varepsilon}\right) d x d t+O_{\varepsilon} .
\end{aligned}
$$

From (3.6) in the first term in the right-hand side of (4.50), and (4.18) for the second term, then we have

$$
\begin{aligned}
& \int_{0}^{T} \int_{\Omega} q_{\varepsilon}(t)\left(\operatorname{div} \varphi+\varepsilon^{-\frac{1}{3}} \operatorname{div}_{\mathrm{y}} \widehat{\varphi}\left(x^{\prime}, \frac{x}{\varepsilon}\right)\right) d x \\
& =\int_{0}^{T} \int_{\Omega} q(t) \operatorname{div} \varphi d x+\int_{0}^{T} \int_{\mathcal{T}^{2} \times \widehat{Q}} \widehat{q}(t) \operatorname{div}_{\mathrm{y}} \widehat{\varphi} d x^{\prime} d y+O_{\varepsilon} .
\end{aligned}
$$

- Fifth term of (4.42). In order to identify the limit of the boundary term, we write

$$
\begin{aligned}
& \beta \int_{0}^{T} \phi(t) \int_{\Gamma_{\varepsilon}} w_{\varepsilon}(t) \cdot \varphi d \mathcal{H}^{2} \\
& =\beta \int_{0}^{T} \phi(t) \int_{\mathcal{T}^{2}} w_{\varepsilon}(t)\left(t, x^{\prime},-a_{\varepsilon} \Psi\left(\frac{x^{\prime}}{\varepsilon}\right)\right) \cdot \varphi\left(x^{\prime},-a_{\varepsilon} \Psi\left(\frac{x^{\prime}}{\varepsilon}\right)\right) \sqrt{1+\left(\frac{a_{\varepsilon}}{\varepsilon}\right)^{2}\left|\nabla \Psi\left(\frac{x^{\prime}}{\varepsilon}\right)\right|^{2}} d x^{\prime} .
\end{aligned}
$$

The inequality

$$
\begin{aligned}
& \int_{\mathcal{T}^{2}}\left|w_{\varepsilon}\left(t, x^{\prime},-a_{\varepsilon} \Psi\left(\frac{x^{\prime}}{\varepsilon}\right)\right)-w_{\varepsilon}\left(t, x^{\prime}, 0\right)\right|^{3} d x^{\prime}=\int_{\mathcal{T}^{2}}\left|\int_{-a_{\varepsilon} \Psi\left(\frac{x^{\prime}}{\varepsilon}\right)}^{0} \partial_{3} w_{\varepsilon}\left(t, x^{\prime}, s\right) d s\right|^{3} d x^{\prime} \\
& \leq C a_{\varepsilon}^{2} \int_{\Omega_{\varepsilon}}\left|\partial_{3} w_{\varepsilon}(t)\right|^{3} d x=O_{\varepsilon},
\end{aligned}
$$

the strong convergence of $w_{\varepsilon}\left(t, x^{\prime}, 0\right)$ to $w\left(t, x^{\prime}, 0\right)$ in $L^{3}(] 0, T\left[, L^{3}\left(\mathcal{T}^{2}, \mathbb{R}^{3}\right)\right)$ and the fact that $a_{\varepsilon} / \varepsilon$ tends to zero, imply

$$
\beta \int_{0}^{T} \phi(t) \int_{\Gamma_{\varepsilon}} w_{\varepsilon}(t) \cdot \varphi d \mathcal{H}^{2} d t=\beta \int_{0}^{T} \phi(t) \int_{\Gamma} w(t) \cdot \varphi d \mathcal{H}^{2} d t+O_{\varepsilon} .
$$

- Last term of (4.42). Since $\left|\Omega_{\varepsilon} \backslash \Omega\right| \rightarrow 0$, we have

$$
\int_{0}^{T} \phi(t) \int_{\Omega_{\varepsilon}} \bar{f} \cdot \varphi d x d t=\int_{0}^{T} \phi(t) \int_{\Omega} \bar{f} \cdot \varphi d x d t+O_{\varepsilon} .
$$

Therefore, substituting (4.43), (4.44), (4.49), (4.51), (4.54) and (4.55) in (4.42), we get that the limit problem is equivalent to the following one

$$
\begin{aligned}
& \int_{0}^{T} \int_{\Omega} \partial_{t} w(t) \cdot \varphi d x d t+\int_{0}^{T} \int_{\Omega}(w(t) \cdot \nabla) w(t) \varphi(t) d x d t \\
& +\int_{0}^{T} \int_{\Omega} \xi(t): D \varphi(t) d x d t+\int_{0}^{T} \int_{\Omega} q(t) \operatorname{div} \varphi(t) d x d t \\
& +\int_{0}^{T} \int_{\mathcal{T}^{2} \times \widehat{Q}} \widehat{\xi}(t): D_{y} \widehat{\varphi}(t) d x^{\prime} d y d t+\int_{0}^{T} \int_{\mathcal{T}^{2} \times \widehat{Q}} \widehat{q}(t) \operatorname{div}_{\mathrm{y}} \widehat{\varphi}(t) d x^{\prime} d y d t \\
& +\beta \int_{0}^{T} \int_{\Gamma} w(t) \cdot \varphi(t) d \mathcal{H}^{2} d t=\int_{0}^{T} \int_{\Omega} \bar{f} \cdot \varphi(t) d x d t
\end{aligned}
$$


for every $\varphi \in C_{c}^{1}\left([0, T] \times \Lambda, \mathbb{R}^{3}\right), \widehat{\varphi} \in C_{c}^{1}\left([0, T] \times \mathcal{T}^{2}, C_{\sharp}^{1}\left(\widehat{Q}, \mathbb{R}^{3}\right)\right)$, with $D_{y} \widehat{\varphi}\left(t, x^{\prime}, y\right)=0$ a.e. in $\left\{y_{3}>M\right\}$, for some $M>0$, and such that (4.37) is satisfied.

By density, this equality holds true for every $\varphi \in L^{3}(] 0, T\left[, W^{1,3}\left(\Omega, \mathbb{R}^{3}\right)\right)$, and every $\widehat{\varphi} \in L^{3}(] 0, T\left[, L^{3}\left(\mathcal{T}^{2}, \mathcal{V}^{3}\right)\right)$ such that

$$
\begin{gathered}
\varphi=0 \text { on }] 0, T[\times(\partial \Omega \backslash \Gamma), \\
\left.\varphi_{3}\left(t, x^{\prime}, 0\right)=0, \quad \widehat{\varphi}_{3}\left(t, x^{\prime}, y^{\prime}, 0\right)=-\lambda \nabla \Psi\left(y^{\prime}\right) \cdot \varphi^{\prime}\left(t, x^{\prime}, 0\right), \quad \text { a.e. }\left(t, x^{\prime}, y^{\prime}\right) \in\right] 0, T\left[\times \mathcal{T}^{2} \times Y^{\prime} .\right.
\end{gathered}
$$

Step 2. Previous property. Let us prove that we have

$$
\begin{aligned}
& \lim _{\varepsilon \rightarrow 0}\left(\int_{0}^{T} \int_{\Omega_{\varepsilon}} S\left(D w_{\varepsilon}(t)\right): D w_{\varepsilon}(t) d x d t+\int_{0}^{T} \int_{\Gamma_{\varepsilon}}\left|w_{\varepsilon}(t)\right|^{2} d \sigma d t\right) \\
& =\int_{0}^{T} \int_{\Omega} \xi(t): D w(t) d x d t+\int_{0}^{T} \int_{\mathcal{T}^{2} \times \widehat{Q}} \widehat{\xi}(t): D_{y} \widehat{w}(t) d x^{\prime} d y d t+\int_{0}^{T} \int_{\Gamma}|w(t)|^{2} d \sigma d t .
\end{aligned}
$$

For this purpose, we take $w_{\varepsilon}$ as test function in (3.4). This gives

$$
\begin{aligned}
& \int_{0}^{T} \int_{\Omega_{\varepsilon}} S\left(D w_{\varepsilon}(t)\right): D w_{\varepsilon}(t) d x d t+\beta \int_{0}^{T} \int_{\Gamma_{\varepsilon}}\left|w_{\varepsilon}(t)\right|^{2} d \sigma d t \\
& =\int_{0}^{T} \int_{\Omega_{\varepsilon}} \bar{f} \cdot w_{\varepsilon}(t) d x d t-\int_{0}^{T} \int_{\Omega_{\varepsilon}} \partial_{t} w_{\varepsilon}(t) \cdot w_{\varepsilon}(t) d x d t
\end{aligned}
$$

Let us pass to the limit in each term of this equality:

For the first term on the right-hand side of (4.58), using that $w_{\varepsilon}$ converges to $w$ weakly in $L^{3}(] 0, T\left[, W^{1,3}\left(\Omega, \mathbb{R}^{3}\right)\right)$ and $\left|\Omega_{\varepsilon} \backslash \Omega\right| \rightarrow 0$, we have

$$
\int_{0}^{T} \int_{\Omega_{\varepsilon}} \bar{f} \cdot w_{\varepsilon}(t) d x d t=\int_{0}^{T} \int_{\Omega} \bar{f} \cdot w(t) d x d t+O_{\varepsilon}
$$

For the second term, from the weak convergence of $\partial_{t} w_{\varepsilon}$ to $\partial_{t} w$ and the strong on of $w_{\varepsilon}$ to $w$, respectively in $L^{2}(] 0, T\left[, L^{2}\left(\Omega, \mathbb{R}^{3}\right)\right)$, we have

$$
\int_{0}^{T} \int_{\Omega_{\varepsilon}} \partial_{t} w_{\varepsilon}(t) \cdot w_{\varepsilon}(t) d x d t=\int_{0}^{T} \int_{\Omega} \partial_{t} w(t) \cdot w(t) d x d t+O_{\varepsilon} .
$$

Therefore, we have then proved that

$$
\int_{0}^{T} \int_{\Omega_{\varepsilon}} S\left(D w_{\varepsilon}\right): D w_{\varepsilon} d x d t+\int_{0}^{T} \int_{\Gamma_{\varepsilon}}\left|w_{\varepsilon}\right|^{2} d \sigma d t=\int_{0}^{T} \int_{\Omega} \bar{f} \cdot \varphi d x d t+\int_{0}^{T} \int_{\Omega} \partial_{t} w \cdot w d x d t+O_{\varepsilon} .
$$

Using now $w$ (the weak limit of $w_{\varepsilon}$ ) and $D_{y} \widehat{w}$ (the weak limit of $\varepsilon^{-\frac{2}{3}} D_{y} \widehat{w}_{\varepsilon}$ ) as test functions in (4.56), we then deduce property (4.57).

Step 3. Identification of $\xi$ and $\widehat{\xi}$. For this, we consider $\eta \in C_{c}^{1}\left([0, T] \times \Lambda, \mathbb{R}^{3}\right)$ and $\widehat{\eta} \in C_{c}^{1}([0, T] \times$ $\left.\mathcal{T}^{2}, C_{\#}^{1}\left(\widehat{Q}, \mathbb{R}^{3}\right)\right)$, with $D_{y} \widehat{\eta}\left(t, x^{\prime}, y\right)=0$ a.e. in $\left\{y_{3}>M\right\}$, for some $M>0$. Also, we consider a sequence $s_{\varepsilon}>0$, such that

$$
\lim _{\varepsilon \rightarrow 0} s_{\varepsilon}=0, \quad \lim _{\varepsilon \rightarrow 0} \frac{s_{\varepsilon}}{\varepsilon}=+\infty .
$$


Using the Hölder inequality and applying the change of variables $y=x / \varepsilon$, we prove

$$
\begin{aligned}
& \int_{\left\{x_{3}>s_{\varepsilon}\right\}}\left|\varepsilon^{-\frac{1}{3}} D_{y} \widehat{\eta}\left(x^{\prime}, \frac{x}{\varepsilon}\right)\right|^{3} d x \\
& =\sum_{k^{\prime} \in \mathbb{Z}^{2}} \int_{C_{\varepsilon}^{k^{\prime}}} \int_{Y^{\prime}} \int_{\frac{s_{\varepsilon}}{\varepsilon}}^{\frac{1}{\varepsilon}}\left|D_{y} \widehat{\eta}\left(t, x^{\prime}, y\right)\right|^{3} d y_{3} d y^{\prime} d x^{\prime}+O_{\varepsilon} \\
& \left.=\int_{\omega} \int_{Y^{\prime}} \int_{\frac{s_{\varepsilon}}{\varepsilon}}^{+\infty}\left|D \widehat{\eta}\left(t, s^{\prime}, y\right)\right|^{3} d y_{3} d y^{\prime} d s^{\prime}=O_{\varepsilon}, \quad \text { a.e. on }\right] 0, T[
\end{aligned}
$$

and on the contrary, we get

$$
\left.\int_{\left\{0<x_{3}<s_{\varepsilon}\right\}}|D \eta(t)|^{3} d x d t=O_{\varepsilon}, \quad \text { a.e. on }\right] 0, T[.
$$

- Behaviour of the operator far away from $\Gamma$ : using the monotonicity property of the operator $S(\cdot)$ (see Lemma 9 in [19]), we have

$$
\int_{0}^{T} \int_{\left\{x_{3}>s_{\varepsilon}\right\}}\left(S\left(D w_{\varepsilon}(t)\right)-S(D \eta(t))\right):\left(D w_{\varepsilon}(t)-D \eta(t)\right) d x d t \geq 0 .
$$

Developping the left-hand side, we get

$$
\begin{aligned}
& \int_{0}^{T} \int_{\left\{x_{3}>s_{\varepsilon}\right\}} S\left(D w_{\varepsilon}(t)\right): D w_{\varepsilon}(t) d x d t-\int_{0}^{T} \int_{\left\{x_{3}>s_{\varepsilon}\right\}} S\left(D w_{\varepsilon}(t)\right): D \eta(t) d x d t \\
& -\int_{0}^{T} \int_{\left\{x_{3}>s_{\varepsilon}\right\}} S(D \eta(t)):\left(D w_{\varepsilon}(t)-D \eta(t)\right) d x d t \geq 0 .
\end{aligned}
$$

Passing to the limit as $\varepsilon \rightarrow 0$, thanks to the weak convergence of $D w_{\varepsilon}$ to $D w$ in $L^{3}(] 0, T\left[, L^{3}\left(\Omega, \mathbb{R}^{9}\right)\right)$, and $S\left(D w_{\varepsilon}\right)$ to $\xi$ in $L^{\frac{3}{2}}(] 0, T\left[, L^{\frac{3}{2}}\left(\Omega, \mathbb{R}^{9}\right)\right)$, we easily get

$$
\begin{aligned}
\lim _{\varepsilon \rightarrow 0} \int_{0}^{T} \int_{\left\{x_{3}>s_{\varepsilon}\right\}} S\left(D w_{\varepsilon}(t)\right): D w_{\varepsilon}(t) d x d t \\
\quad-\int_{0}^{T} \int_{\Omega} \xi(t): D \eta(t) d x d t-\int_{0}^{T} \int_{\Omega} S(D w(t)):(D w(t)-D \eta(t)) d x d t \geq 0 .
\end{aligned}
$$

- Behaviour of the operator near $\Gamma$ : using the monotonicity property of the operator $S(\cdot)$, it holds

$$
\int_{0}^{T} \int_{\left\{0<x_{3}<s_{\varepsilon}\right\}}\left(S\left(D w_{\varepsilon}(t)\right)-S\left(\varepsilon^{-\frac{1}{3}} D_{y} \widehat{\eta}\left(t, x^{\prime}, \frac{x}{\varepsilon}\right)\right)\right):\left(D w_{\varepsilon}(t)-\varepsilon^{-\frac{1}{3}} D_{y} \widehat{\eta}\left(t, x^{\prime}, \frac{x}{\varepsilon}\right)\right) d x d t \geq 0 .
$$

Developping the left-hand side, we get

$$
\begin{aligned}
& \int_{0}^{T} \int_{\left\{0<x_{3}<s_{\varepsilon}\right\}} S\left(D w_{\varepsilon}(t)\right): D w_{\varepsilon}(t) d x d t-\int_{0}^{T} \int_{\left\{0<x_{3}<s_{\varepsilon}\right\}} S\left(D w_{\varepsilon}(t)\right):\left(\varepsilon^{-\frac{1}{3}} D_{y} \widehat{\eta}\left(t, x^{\prime}, \frac{x}{\varepsilon}\right)\right) d x d t \\
& -\int_{0}^{T} \int_{\left\{0<x_{3}<s_{\varepsilon}\right\}} S\left(\varepsilon^{-\frac{1}{3}} D_{y} \widehat{\eta}\left(t, x^{\prime}, \frac{x}{\varepsilon}\right)\right):\left(D w_{\varepsilon}(t)-\varepsilon^{-\frac{1}{3}} D_{y} \widehat{\eta}\left(t, x^{\prime}, \frac{x}{\varepsilon}\right)\right) d x d t \geq 0 .
\end{aligned}
$$


Applying the change of variables (4.16), thanks to the weak convergence of $\varepsilon^{-\frac{2}{3}} D_{y} \widehat{w}_{\varepsilon}$ to $D_{y} \widehat{w}$ in $L^{3}(] 0, T\left[, L^{3}\left(\mathcal{T}^{2} \times\right.\right.$ $\left.\left.\widehat{Q}, \mathbb{R}^{9}\right)\right)$, and $S\left(\varepsilon^{-\frac{2}{3}} D_{y} \widehat{w}_{\varepsilon}\right)$ to $\widehat{\xi}$ in $L^{\frac{3}{2}}(] 0, T\left[L^{\frac{3}{2}}\left(\mathcal{T}^{2} \times \widehat{Q}, \mathbb{R}^{9}\right)\right)$, we obtain

$$
\begin{aligned}
& \int_{0}^{T} \int_{\left\{0<x_{3}<s_{\varepsilon}\right\}} S\left(D w_{\varepsilon}(t)\right):\left(\varepsilon^{-\frac{1}{3}} D_{y} \widehat{\eta}\left(t, x^{\prime}, \frac{x}{\varepsilon}\right)\right) d x d t \\
& =\varepsilon^{\frac{2}{3}} \int_{0}^{T} \int_{\mathcal{T}^{2} \times \widehat{Q}_{s_{\varepsilon}}} S\left(\varepsilon^{-1} D_{y} \widehat{w}_{\varepsilon}(t)\right): D_{y} \widehat{\eta}(t) d x^{\prime} d y d t+O_{\varepsilon} \\
& =\varepsilon \int_{0}^{T} \int_{\mathcal{T}^{2} \times \widehat{Q}_{s_{\varepsilon}}} D_{y} \widehat{w}_{\varepsilon}(t): D_{y} \widehat{\eta}(t) d x^{\prime} d y d t+\int_{0}^{T} \int_{\mathcal{T}^{2} \times \widehat{Q}_{s_{\varepsilon}}} \widehat{S}\left(\varepsilon^{-\frac{2}{3}} D_{y} \widehat{w}_{\varepsilon}(t)\right): D_{y} \widehat{\eta}(t) d x^{\prime} d y d t+O_{\varepsilon} \\
& =\int_{0}^{T} \int_{\mathcal{T}^{2} \times \widehat{Q}} \widehat{\xi}(t): D_{y} \widehat{\eta}(t) d x^{\prime} d y d t+O_{\varepsilon},
\end{aligned}
$$

and

$$
\begin{aligned}
& \int_{0}^{T} \int_{\left\{0<x_{3}<s_{\varepsilon}\right\}} S\left(\varepsilon^{-\frac{1}{3}} D_{y} \widehat{\eta}\left(t, x^{\prime}, \frac{x}{\varepsilon}\right)\right):\left(D w_{\varepsilon}(t)-\varepsilon^{-\frac{1}{3}} D_{y} \widehat{\eta}\left(t, x^{\prime}, \frac{x}{\varepsilon}\right)\right) d x d t \\
& =\varepsilon^{-\frac{1}{3}} \int_{0}^{T} \int_{\left\{0<x_{3}<s_{\varepsilon}\right\}} D_{y} \widehat{\eta}\left(t, x^{\prime}, \frac{x}{\varepsilon}\right):\left(D w_{\varepsilon}(t)-\varepsilon^{-\frac{1}{3}} D_{y} \widehat{\eta}\left(t, x^{\prime}, \frac{x}{\varepsilon}\right)\right) d x d t \\
& +\varepsilon^{-\frac{2}{3}} \int_{0}^{T} \int_{\left\{0<x_{3}<s_{\varepsilon}\right\}} \widehat{S}\left(D_{y} \widehat{\eta}\left(t, x^{\prime}, \frac{x}{\varepsilon}\right)\right):\left(D w_{\varepsilon}(t)-\varepsilon^{-\frac{1}{3}} D_{y} \widehat{\eta}\left(t, x^{\prime}, \frac{x}{\varepsilon}\right)\right) d x d t \\
& =\varepsilon^{\frac{2}{3}} \int_{0}^{T} \int_{\mathcal{T}^{2} \times \widehat{Q}_{s_{\varepsilon}}} D_{y} \widehat{\eta}(t):\left(\varepsilon^{-1} D_{y} \widehat{w}_{\varepsilon}(t)-\varepsilon^{-\frac{1}{3}} D_{y} \widehat{\eta}(t)\right) d x^{\prime} d y d t \\
& +\varepsilon^{\frac{1}{3}} \int_{0}^{T} \int_{\mathcal{T}^{2} \times \widehat{Q}_{s_{\varepsilon}}} \widehat{S}\left(D_{y} \widehat{\eta}(t)\right):\left(\varepsilon^{-1} D_{y} \widehat{w}_{\varepsilon}(t)-\varepsilon^{-\frac{1}{3}} D_{y} \widehat{\eta}(t)\right) d x^{\prime} d y d t+O_{\varepsilon} \\
& =\varepsilon^{\frac{1}{3}} \int_{0}^{T} \int_{\mathcal{T}^{2} \times \widehat{Q}_{s_{\varepsilon}}} D_{y} \widehat{\eta}(t):\left(\varepsilon^{-\frac{2}{3}} D_{y} \widehat{w}_{\varepsilon}(t)-D_{y} \widehat{\eta}(t)\right) d x^{\prime} d y d t \\
& +\int_{0}^{T} \int_{\mathcal{T}^{2} \times \widehat{Q}_{s_{\varepsilon}}} \widehat{S}\left(D_{y} \widehat{\eta}(t)\right):\left(\varepsilon^{-\frac{2}{3}} D_{y} \widehat{w}_{\varepsilon}(t)-D_{y} \widehat{\eta}(t)\right) d x^{\prime} d y d t+O_{\varepsilon} \\
& =\int_{0}^{T} \int_{\mathcal{T}^{2} \times \widehat{Q}} \widehat{S}\left(D_{y} \widehat{\eta}(t)\right):\left(D_{y} \widehat{w}(t)-D_{y} \widehat{\eta}(t)\right) d x^{\prime} d y d t+O_{\varepsilon} .
\end{aligned}
$$

Passing to the limit in every terms of (4.63), taking into account the computations above, we get

$$
\begin{aligned}
& \lim _{\varepsilon \rightarrow 0} \int_{0}^{T} \int_{\left\{0<s_{\varepsilon}<\varepsilon\right\}} S\left(D w_{\varepsilon}(t)\right): D w_{\varepsilon}(t) d x d t \\
& \left.-\int_{0}^{T} \int_{\mathcal{T}^{2} \times \widehat{Q}} \widehat{\xi}(t): D_{y} \widehat{\eta}(t) d x^{\prime} d y d t-\int_{0}^{T} \int_{\mathcal{T}^{2} \times \widehat{Q}} \widehat{S}\left(D_{y} \widehat{w}(t)\right)\right):\left(D_{y} \widehat{w}(t)-D_{y} \widehat{\eta}(t)\right) d x^{\prime} d y d t \geq 0 .
\end{aligned}
$$

- Adding (4.62), (4.64), the boundary integral $\int_{0}^{T} \int_{\Gamma_{\varepsilon}}\left|w_{\varepsilon}(t)-w(t)\right|^{2} d \sigma d t \geq 0$, and taking into account that

$$
\lim _{\varepsilon \rightarrow 0} \int_{0}^{T} \int_{\Omega_{\varepsilon} \backslash \Omega} S\left(D w_{\varepsilon}(t)\right): D w_{\varepsilon}(t) d x d t=0,
$$


we get

$$
\begin{aligned}
& \lim _{\varepsilon \rightarrow 0}\left(\int_{0}^{T} \int_{\Omega_{\varepsilon}} S\left(D w_{\varepsilon}(t)\right): D w_{\varepsilon}(t) d x d t+\int_{0}^{T} \int_{\Gamma_{\varepsilon}}\left|w_{\varepsilon}(t)-w(t)\right|^{2} d \sigma d t\right) \\
& -\int_{0}^{T} \int_{\Omega} \xi(t): D \eta(t) d x d t-\int_{0}^{T} \int_{\Omega} S(D w(t)):(D w(t)-D \eta(t)) d x d t \\
& \left.-\int_{0}^{T} \int_{\mathcal{T}^{2} \times \widehat{Q}} \widehat{\xi}(t): D_{y} \widehat{\eta}(t) d x^{\prime} d y d t-\int_{0}^{T} \int_{\mathcal{T}^{2} \times \widehat{Q}} \widehat{S}\left(D_{y} \widehat{w}(t)\right)\right):\left(D_{y} \widehat{w}(t)-D_{y} \widehat{\eta}(t)\right) d x^{\prime} d y d t \geq 0 .
\end{aligned}
$$

Using property (4.57), and taking into account that

$$
\begin{aligned}
\int_{0}^{T} \int_{\Gamma_{\varepsilon}}\left|w_{\varepsilon}(t)-w(t)\right|^{2} d \sigma d t= & \int_{0}^{T} \int_{\Gamma_{\varepsilon}}\left|w_{\varepsilon}(t)\right|^{2} d \sigma d t+\int_{0}^{T} \int_{\Gamma_{\varepsilon}}|w(t)|^{2} d \sigma d t \\
& -2 \int_{0}^{T} \int_{\Gamma_{\varepsilon}} w_{\varepsilon}(t) \cdot w(t) d \sigma d t \\
= & \int_{0}^{T} \int_{\Gamma_{\varepsilon}}\left|w_{\varepsilon}(t)\right|^{2} d \sigma d t-\int_{0}^{T} \int_{\Gamma}|w(t)|^{2} d \sigma d t+O_{\varepsilon},
\end{aligned}
$$

we have

$$
\begin{aligned}
& \int_{0}^{T} \int_{\Omega}(\xi(t)-S(D w(t))):(D w(t)-D \eta(t)) d x d t \\
& \quad+\int_{0}^{T} \int_{\mathcal{T}^{2} \times \widehat{Q}}\left(\widehat{\xi}(t)-\widehat{S}\left(D_{y} \widehat{w}(t)\right)\right):\left(D_{y} \widehat{w}(t)-D_{y} \widehat{\eta}(t)\right) d x^{\prime} d y d t \geq 0 .
\end{aligned}
$$

- Identification of $\xi$ : for this, we consider $\eta=w-\tau \zeta, \forall \tau>0$ with $\zeta \in C_{c}^{1}\left([0, T] \times \Lambda, \mathbb{R}^{3}\right)$ and $\widehat{\eta}=\widehat{w}$ in (4.65), which gives

$$
\int_{0}^{T} \int_{\Omega}(\xi(t)-S(D w(t)-\tau D \zeta(t))): D \zeta(t) d x d t \geq 0
$$

Therefore this inequality, due to the fact that

$$
S(D w-\tau D \zeta): D \zeta=S(D w): D \zeta+(S(D w-\tau D \zeta)-S(D w)): D \zeta \leq S(D w): D \zeta
$$

is valid for an arbitrary function $\zeta$ and, after $\tau \rightarrow 0$, the equality sign hold. Therefore,

$$
\xi=S(D w)
$$

- Identification of $\widehat{\xi}$ : for this we consider now $\widehat{\eta}=\widehat{w}-\tau \widehat{\zeta}, \forall \tau>0$ with $\widehat{\zeta} \in C_{c}^{1}\left([0, T] \times \mathcal{T}^{2}, C_{\#}^{1}\left(\widehat{Q}, \mathbb{R}^{3}\right)\right)$ and $D_{y} \widehat{\zeta}\left(t, x^{\prime}, y\right)=0$ a.e. in $\left\{y_{3}>M\right\}$, for some $M>0$, and $\eta=u$ in (4.65), which gives

$$
\int_{0}^{T} \int_{\mathcal{T}^{2} \times \widehat{Q}}\left(\widehat{\xi}(t)-\widehat{S}\left(D_{y} \widehat{w}(t)-\tau D_{y} \widehat{\zeta}(t)\right)\right): D \zeta(t) d x d t \geq 0
$$

Therefore this inequality, due to the fact that

$$
S\left(D_{y} \widehat{w}-\tau D_{y} \widehat{\zeta}\right): D_{y} \widehat{\zeta}=S\left(D_{y} \widehat{w}\right): D_{y} \widehat{\zeta}+\left(S\left(D_{y} \widehat{w}-\tau D_{y} \widehat{\zeta}\right)-S\left(D_{y} \widehat{w}\right)\right): D_{y} \widehat{\zeta} \leq S\left(D_{y} \widehat{w}\right): D_{y} \widehat{\zeta}
$$


is valid for an arbitrary function $\widehat{\zeta}$ and, after $\tau \rightarrow 0$, the equality sign hold. Therefore

$$
\widehat{\xi}=\widehat{S}\left(D_{y} \widehat{w}\right) .
$$

Step 4. Boundary layer system satisfied by $\widehat{w}$. From the previous steps, the limit problem (4.56) reads

$$
\begin{aligned}
& \int_{0}^{T} \partial_{t} w(t) \cdot \varphi(t) d x d t+\int_{0}^{T} \int_{\Omega}(w(t) \cdot \nabla) w(t) \varphi(t) d x d t \\
& +\int_{0}^{T} \int_{\Omega} S(D w(t)): D \varphi(t) d x d t-\int_{0}^{T} \int_{\Omega} q(t) \operatorname{div} \varphi(t) d x d t \\
& +\int_{0}^{T} \int_{\omega \times \widehat{Q}} \widehat{S}\left(D_{y} \widehat{w}(t)\right): D_{y} \widehat{\varphi}(t) d x^{\prime} d y d t-\int_{0}^{T} \int_{\omega \times \widehat{Q}} \widehat{q}(t) \operatorname{div}_{\mathrm{y}} \widehat{\varphi}(t) d x^{\prime} d y d t \\
& +\beta \int_{0}^{T} \int_{\Gamma} w(t) \varphi(t) d \sigma d t=\int_{0}^{T} \int_{\Omega} \bar{f} \cdot \varphi(t) d x d t
\end{aligned}
$$

for every $\varphi \in L^{3}(] 0, T\left[, W^{1,3}\left(\Omega, \mathbb{R}^{3}\right)\right)$, and every $\widehat{\varphi} \in L^{3}(] 0, T\left[, L^{3}\left(\mathcal{T}^{2}, \mathcal{V}^{3}\right)\right)$ such that

$$
\begin{gathered}
\varphi=0 \text { on }] 0, T\left[\times \Gamma_{0},\right. \\
\left.\varphi_{3}\left(t, x^{\prime}, 0\right)=0, \quad \widehat{\varphi}_{3}\left(t, x^{\prime}, y^{\prime}, 0\right)=-\lambda \nabla \Psi\left(y^{\prime}\right) \cdot \varphi^{\prime}\left(t, x^{\prime}, 0\right), \quad \text { a.e. }\left(t, x^{\prime}, y^{\prime}\right) \in\right] 0, T\left[\times \mathcal{T}^{2} \times Y^{\prime} .\right.
\end{gathered}
$$

In this step, we will obtain an equation for $w$ eliminating $\widehat{w}$ and $\widehat{q}$ in (4.66). For this purpose, we take $\varphi=0$ in (4.66). This proves that $(\widehat{w}, \widehat{q}) \in L^{3}(] 0, T\left[\times \mathcal{T}^{2}, \mathcal{V}^{3}\right) \times W^{-1, \infty}(] 0, T\left[, L_{\#}^{3 / 2}\left(\mathcal{T}^{2} \times \widehat{Q}_{M}, \mathbb{R}\right)\right)$ is a solution of

$$
\left\{\begin{array}{l}
-\operatorname{div}_{\mathrm{y}} \widehat{S}\left(D_{y} \widehat{w}\right)+\nabla_{y} \widehat{q}=0 \quad \text { in } \mathbb{R}^{2} \times \mathbb{R}^{+}, \\
\operatorname{div}_{\mathrm{y}} \widehat{w}=0 \quad \text { in } \mathbb{R}^{2} \times \mathbb{R}^{+}, \\
\widehat{w}_{3}\left(t, x^{\prime}, y^{\prime}, 0\right)=-\lambda \nabla \Psi\left(y^{\prime}\right) \cdot u^{\prime}\left(t, x^{\prime}, 0\right) \text { on } \mathbb{R}^{2} \times\{0\}, \\
S\left(D_{y} \widehat{w}\right)_{i, 3}=0, i=1,2, \quad \text { on } \mathbb{R}^{2} \times\{0\},
\end{array}\right.
$$

a.e. in $] 0, T\left[\times \mathcal{T}^{2}\right.$. Defining $\left(\widehat{\phi}^{\xi^{\prime}}, \widehat{\pi}^{\xi^{\prime}}\right)$, for every $\xi^{\prime} \in \mathbb{R}^{2}$, by $(3.10)$, we deduce that

$$
\left.\widehat{w}\left(t, x^{\prime}, y\right)=\lambda \widehat{\phi}^{w^{\prime}\left(t, x^{\prime}, 0\right)}(y), \quad \widehat{q}\left(t, x^{\prime}, y\right)=\lambda^{2} \widehat{\pi}^{w^{\prime}\left(t, x^{\prime}, 0\right)}(y), \quad \text { a.e. }\left(t, x^{\prime}, y\right) \in\right] 0, T\left[\times \mathcal{T}^{2} \times \widehat{Q} .\right.
$$

Now, for $\varphi \in L^{3}(] 0, T\left[, W^{1,3}\left(\Omega, \mathbb{R}^{3}\right)\right)$, with $\varphi=0$ on $\partial \Omega \backslash \Gamma, \varphi_{3}=0$ on $] 0, T[\times \Gamma$, we take $\varphi$ and $\widehat{\varphi}\left(t, x^{\prime}, y\right)=\lambda\left(\varphi_{1}\left(t, x^{\prime}, 0\right) \widehat{\phi}^{e_{1}}(y)+\varphi_{2}\left(t, x^{\prime}, 0\right) \widehat{\phi}^{e_{2}}(y)\right)$ as test functions in (4.66). Taking into account (4.68) and definition (3.9) we get

$$
\begin{aligned}
& \int_{0}^{T} \int_{\Omega} \partial_{t} w(t) \cdot \varphi(t) d x d t+\int_{0}^{T} \int_{\Omega}(w(t) \cdot \nabla) w(t) \cdot \varphi(t) d x d t \\
& +\int_{0}^{T} \int_{\Omega} S(D w(t)): D \varphi(t) d x d t-\int_{0}^{T} \int_{\Omega} q(t) \operatorname{div} \varphi d x d t \\
& +\beta \int_{0}^{T} \int_{\mathcal{T}^{2}} w^{\prime}\left(t, x^{\prime}, 0\right) \varphi^{\prime}\left(t, x^{\prime}, 0\right) d x^{\prime} d t+\lambda^{3} \int_{\omega} R\left(w^{\prime}\left(t, x^{\prime}, 0\right)\right) \varphi^{\prime}\left(t, x^{\prime}, 0\right) d x^{\prime} d t \\
& =\int_{0}^{T} \int_{\Omega} \bar{f} \cdot \varphi(t) d x d t .
\end{aligned}
$$


By the arbitrariness of $\varphi$, this proves that $(w, q)$ is a solution of (3.7)-(3.8). Notice that the initial condition $w(0, \cdot)=w^{0}(\cdot)$ in $L^{2}\left(\Omega, \mathbb{R}^{3}\right)$ is a consequence of the continuity of the linear operator $u \in$ $H^{1}(] 0, T\left[, L^{2}\left(\Omega, \mathbb{R}^{3}\right)\right) \rightarrow u(0, \cdot) \in L^{2}\left(\Omega, \mathbb{R}^{3}\right)$, and of the weak convergence of $w_{\varepsilon \mid \Omega}^{0}$ to $w^{0}$ in $L^{2}\left(\Omega, \mathbb{R}^{3}\right)$.

Proof of Corollary (riblets). The idea is to proceed as the proof of Theorem 3.3 by considering that $\partial_{y_{2}} \Psi=0$. In fact, in Step 1, we take a test function $\hat{\varphi}$ in (4.37) satisfying $\widehat{\varphi}_{3}\left(x^{\prime}, y^{\prime}, 0\right)=-\lambda \partial_{y_{1}} \Psi\left(y_{1}\right) \varphi_{1}\left(x^{\prime}, 0\right)$, which leads to a problem (4.67) satisfying the condition $\widehat{w}_{3}\left(t, x^{\prime}, y^{\prime}, 0\right)=-\lambda \partial_{y_{1}} \Psi\left(y_{1}\right) u_{1}\left(t, x^{\prime}, 0\right)$ on $\mathbb{R}^{2} \times$ $\{0\}$.

Defining $\widehat{w}\left(t, x^{\prime}, y\right)=\lambda \widehat{\phi}^{w^{\prime}\left(t, x^{\prime}, 0\right) e_{1}}(y), \widehat{q}\left(t, x^{\prime}, y\right)=\lambda^{2} \widehat{\pi} w^{\prime}\left(t, x^{\prime}, 0\right) e_{1}(y)$, a.e. $\left.\left(t, x^{\prime}, y\right) \in\right] 0, T\left[\times \mathcal{T}^{2} \times \widehat{Q}\right.$, and taking $\widehat{\varphi}\left(t, x^{\prime}, y\right)=\lambda \varphi_{1}\left(t, x^{\prime}, 0\right) \widehat{\phi}^{e_{1}}(y)$ in Step 4 , we can deduce that the effective boundary condition on $\Gamma$ is

$$
\left.-S(D w)_{1,3}+\beta w_{1}+\lambda^{3} R_{1}\left(\left(w_{1}, 0\right)\right)=0 \quad \text { on }\right] 0, T\left[\times \Gamma, \quad-S(D w)_{2,3}+\beta w_{2}=0 \quad \text { on }\right] 0, T[\times \Gamma .
$$

We observe that $R_{1}\left(\left(w_{1}, 0\right)\right)$ can be simplified by taking into account the homogeneity condition $(2.20)$. Thus, if $w_{1}\left(t, x^{\prime}, 0\right) \geq 0$, then $R_{1}\left(\left(w_{1}, 0\right)\right)=w_{1}^{2} R_{1}\left(e_{1}\right)$, whereas if $w_{1}\left(t, x^{\prime}, 0\right)<0, R_{1}\left(\left(w_{1}, 0\right)\right)=$ $w_{1}^{2} R_{1}\left(-e_{1}\right)$. This leads to the formulation (3.11) of the boundary conditions on $\Gamma$.

Proof of Proposition 3.8. The existence and uniqueness of a solution to problem (3.10) results from the theory of monotone operators of J. Leray and J.L. Lions [34,35]. To obtain estimate (3.12), for each $\xi^{\prime} \in \mathbb{R}^{2}$, we introduce a function $G \in(\mathcal{V} / \mathbb{R})^{2} \times \mathcal{V} \times L_{\#}^{\frac{3}{2}}(\widehat{Q}, \mathbb{R})$ satisfying

$$
\operatorname{div}_{\mathrm{y}} G=0 \text { in } \widehat{Q}, \quad G^{\prime}\left(y^{\prime}, 0\right)=0, G_{3}\left(y^{\prime}, 0\right)=-\nabla \Psi\left(y^{\prime}\right) \cdot \xi^{\prime} \quad \text { for a.e. } y^{\prime} \in \mathcal{T}^{2},
$$

and

$$
\left\|D_{y} G\right\|_{L^{3}(\widehat{Q})} \leq C\left|\xi^{\prime}\right|\|\nabla \Psi\|_{L^{3}\left(\mathcal{T}^{2}\right)},
$$

where $C>0$ is a constant. The function $\widehat{\phi}^{\xi^{\prime}}-G$ is an admissible test function for problem (3.10), which yields

$$
\int_{\widehat{Q}} \widehat{S}\left(D_{y} \widehat{\phi}^{\xi^{\prime}}\right):\left(D_{y} \widehat{\phi}^{\xi^{\prime}}-D_{y} G\right) d y=0
$$

Consequently, using properties (4.1) and (4.2), we obtain

$$
\begin{aligned}
\left(C_{s}-\frac{\delta^{2}}{2 \gamma}\right)\left\|D_{y} \widehat{\phi}^{\xi^{\prime}}\right\|_{L^{3}\left(\widehat{Q}, \mathbb{R}^{3}\right)}^{3} \leq \int_{\widehat{Q}} \widehat{S}\left(D_{y} \widehat{\phi}^{\xi^{\prime}}\right): D_{y} \widehat{\phi}^{\xi^{\prime}} d y & =\int_{\widehat{Q}} \widehat{S}\left(D_{y} \widehat{\phi}^{\xi^{\prime}}\right): D_{y} G d y \\
& \leq C\left\|D_{y} \widehat{\phi}^{\xi^{\prime}}\right\|_{L^{3}\left(\widehat{Q}, \mathbb{R}^{3}\right)}^{2}\left\|D_{y} G\right\|_{L^{3}\left(\widehat{Q}, \mathbb{R}^{3}\right)}
\end{aligned}
$$

This yields (3.12). Finally, the exponential decay property (3.13) is proved in [15].

Remark 4.6 (Monotonicity of the friction term introduced by the roughness effect.). Consider the operator $R: \mathbb{R}^{2} \rightarrow \mathbb{R}^{2}, \xi^{\prime} \mapsto\left(R_{1}\left(\xi^{\prime}, R_{2}\left(\xi^{\prime}\right)\right)\right.$, where for $i=1,2, R_{i}$ is defined by (3.9). Then, $R$ satisfies the following monotonicity property: for every $\xi_{1}^{\prime}, \xi_{2}^{\prime} \in \mathbb{R}^{2}$,

$$
\left(R\left(\xi_{1}^{\prime}\right)-R\left(\xi_{2}^{\prime}\right)\right) \cdot\left(\xi_{1}^{\prime}-\xi_{2}^{\prime}\right) \geq 0 .
$$


Proof. Let $\xi_{1}^{\prime}, \xi_{2}^{\prime} \in \mathbb{R}^{2}$. Developing the scalar product and using definition (3.9), we obtain

$$
\begin{aligned}
\left(R\left(\xi_{1}^{\prime}\right)-R\left(\xi_{2}^{\prime}\right)\right) \cdot\left(\xi_{1}^{\prime}-\xi_{2}^{\prime}\right) & =\left(R_{1}\left(\xi_{1}^{\prime}\right)-R_{1}\left(\xi_{2}^{\prime}\right)\right)\left(\xi_{1}^{\prime}-\xi_{2}^{\prime}\right)_{1}+\left(R_{2}\left(\xi_{1}^{\prime}\right)-R_{2}\left(\xi_{2}^{\prime}\right)\right)\left(\xi_{1}^{\prime}-\xi_{2}^{\prime}\right)_{2} \\
& =\int_{\widehat{Q}}\left(\widehat{S}\left(D_{y} \widehat{\phi}^{\xi_{1}^{\prime}}\right)-\widehat{S}\left(D_{y} \widehat{\phi}^{\xi_{2}^{\prime}}\right)\right):\left(\left(\xi_{1}^{\prime}-\xi_{2}^{\prime}\right)_{1} D_{y} \widehat{\phi}^{e_{1}}+\left(\xi_{1}^{\prime}-\xi_{2}^{\prime}\right)_{2} D_{y} \widehat{\phi}^{e_{2}}\right) d y \\
& =\int_{\widehat{Q}}\left(\widehat{S}\left(D_{y} \widehat{\phi}^{\xi_{1}^{\prime}}\right)-\widehat{S}\left(D_{y} \widehat{\phi}^{\xi_{2}^{\prime}}\right)\right): D_{y}\left(\left(\xi_{1}^{\prime}-\xi_{2}^{\prime}\right)_{1} \widehat{\phi}^{e_{1}}+\left(\xi_{1}^{\prime}-\xi_{2}^{\prime}\right)_{2} \widehat{\phi}^{e_{2}}\right) d y
\end{aligned}
$$

Now, defining $G \in(\mathcal{V} / \mathbb{R})^{2} \times \mathcal{V} \times L_{\#}^{\frac{3}{2}}(\widehat{Q}, \mathbb{R})$ as the combination $G=\left(\xi_{1}^{\prime}-\xi_{2}^{\prime}\right)_{1} \widehat{\phi}^{e_{1}}+\left(\xi_{1}^{\prime}-\xi_{2}^{\prime}\right)_{2} \widehat{\phi}^{e_{2}}$, we notice that $G$ satisfies the same boundary condition as the difference $\widehat{\phi}^{\xi_{1}^{\prime}}-\widehat{\phi}^{\xi_{1}^{\prime}}$, namely,

$$
G_{3}\left(y^{\prime}, 0\right)=-\nabla \Psi\left(y^{\prime}\right) \cdot\left(\xi_{1}^{\prime}-\xi_{2}^{\prime}\right)=\widehat{\phi}_{3}^{\xi_{1}^{\prime}}\left(y^{\prime}, 0\right)-\widehat{\phi}_{3}^{\xi_{2}^{\prime}}\left(y^{\prime}, 0\right) \quad \text { for a.e. } y^{\prime} \in \mathcal{T}^{2} \text {. }
$$

Consequently, $G-\left(\widehat{\phi}^{\xi_{1}^{\prime}}-\widehat{\phi}^{\xi_{1}^{\prime}}\right)$ is an admissible test function for problem (3.10), with $\xi^{\prime} \in\left\{\xi_{1}^{\prime}, \xi_{2}^{\prime}\right\}$. Arguing as in the proof of Proposition 3.8, we further deduce that

$$
\begin{aligned}
\left(R\left(\xi_{1}^{\prime}\right)-R\left(\xi_{2}^{\prime}\right)\right) \cdot\left(\xi_{1}^{\prime}-\xi_{2}^{\prime}\right) & =\int_{\widehat{Q}}\left(\widehat{S}\left(D_{y} \widehat{\phi}^{\xi_{1}^{\prime}}\right)-\widehat{S}\left(D_{y} \widehat{\phi}^{\xi_{2}^{\prime}}\right)\right): D_{y}\left(\widehat{\phi}^{\xi_{1}^{\prime}}-\widehat{\phi}^{\xi_{2}^{\prime}}\right) d y \\
& =\int_{\widehat{Q}}\left(\widehat{S}\left(D_{y} \widehat{\phi}^{\xi_{1}^{\prime}}\right)-\widehat{S}\left(D_{y} \widehat{\phi}^{\xi_{2}^{\prime}}\right)\right):\left(D_{y} \widehat{\phi}^{\xi_{1}^{\prime}}-D_{y} \widehat{\phi}^{\prime}\right) d y
\end{aligned}
$$

which is nonnegative by monotonicity of $\widehat{S}$.

\section{Numerical results}

In this section, we investigate numerically the behaviour of the solution of the LES model (2.14)-(2.16), in the case where the lower plate is composed of riblets, which are very commonly used in experimental and theoretical studies of the roughness effects on viscous flows, in particular for turbulent regimes (see, for instance, $[8,22,25])$. In that case, in our framework, the sequence of functions $\Psi_{\varepsilon}$ describing the geometry of the rough wall, is defined by

$$
\Psi_{\varepsilon}\left(x^{\prime}\right)=a_{\varepsilon} \Psi\left(x_{1} / \varepsilon\right), \quad x^{\prime} \in \mathcal{T}^{2},
$$

where $\Psi$ is a given, positive 1-periodic and regular function of variable $x_{1}$, and where the sequence $\left(a_{\varepsilon}\right)_{\varepsilon>0}$ satisfies assumptions (2.1). By Corollary 3.6, the effective velocity and pressure $w, q$ satisfy the limit system (3.7), completed with the boundary conditions (3.11) on the (idealized) flat lower plate $\Gamma$, that express the effect of the asperities on the LES model, at main order in $\varepsilon$.

In order to stress the influence of the parameter $\lambda$, defined by (3.5), we simulate stationary solutions of the limit system (3.7), which allows us to aggregate numerous numerical results associated with a wide spectrum of values of $\lambda$. In the case of the riblet geometry described by (5.1), we seek the velocity $w$ and the pressure $q$ satisfying the following effective system:

$$
\left\{\begin{aligned}
(w \cdot \nabla) w-\operatorname{div}(S(D w))+\nabla q & =\bar{f} & & \text { in } \Omega, \\
\operatorname{div} w & =0 & & \text { in } \Omega, \\
w & =0 & & \text { on } \Gamma^{0}, \\
w_{3} & =0 & & \text { on } \Gamma, \\
-S(D w)_{1,3}+\beta w_{1}+\lambda^{3} w_{1}^{2} R_{1}\left(\operatorname{sgn}\left(w_{1}\right) e_{1}\right) & =0 & & \text { on } \Gamma, \\
-S(D w)_{2,3}+\beta w_{2} & = & & \text { on } \Gamma .
\end{aligned}\right.
$$


To investigate the influence of the shape of the riblets on the effective flow, we will focus on three different profiles for the function $\Psi$, that correspond to the $V$-shaped, the $U$-shaped and the blade-shaped riblets. These shapes are widely used in the engineering literature, and are drawn in Table 1. Notice that, for each profile, we have applied a parabolic regularization in the vicinity of each singular point, obtaining functions $\Psi$ of class $C^{2}$. Table 2 gives an example of transversal sections of ribbed walls $\Gamma_{\varepsilon}$, associated with those different profiles $\Psi$ by the definition

$$
\Gamma_{\varepsilon}=\left\{\left(x_{1}, x_{2},-a_{\varepsilon} \Psi\left(x_{1} / \varepsilon\right)\right),\left(x_{1}, x_{2}\right) \in \mathcal{T}^{2}\right\}
$$

We stress that, in the case of riblets, according to boundary conditions (3.11), one needs only to compute the coefficient $R_{1}\left(\xi^{\prime}\right)$ for $\xi^{\prime}=e_{1}$ and $\xi^{\prime}=-e_{1}$, and to have access to the sign of $w_{1}\left(x^{\prime}, 0\right)$, to define the boundary condition at point $x^{\prime} \in \mathcal{T}^{2}$. Let us first address the numerical approximation of coefficients $R_{1}\left(e_{1}\right), R_{1}\left(-e_{1}\right)$.
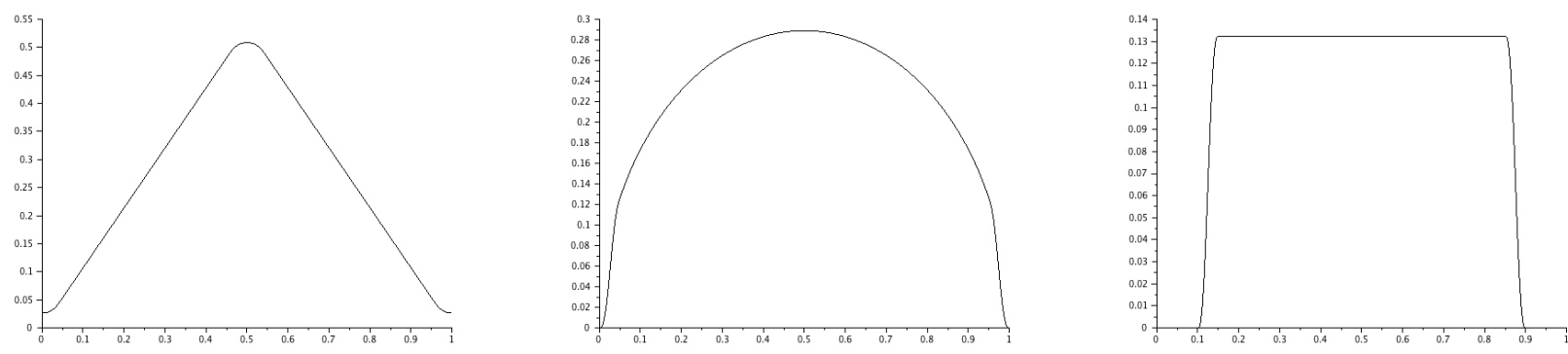

Figure 1: Functions $\Psi$ associated with $V$-shaped, $U$-shaped and blade riblets (from left to right).
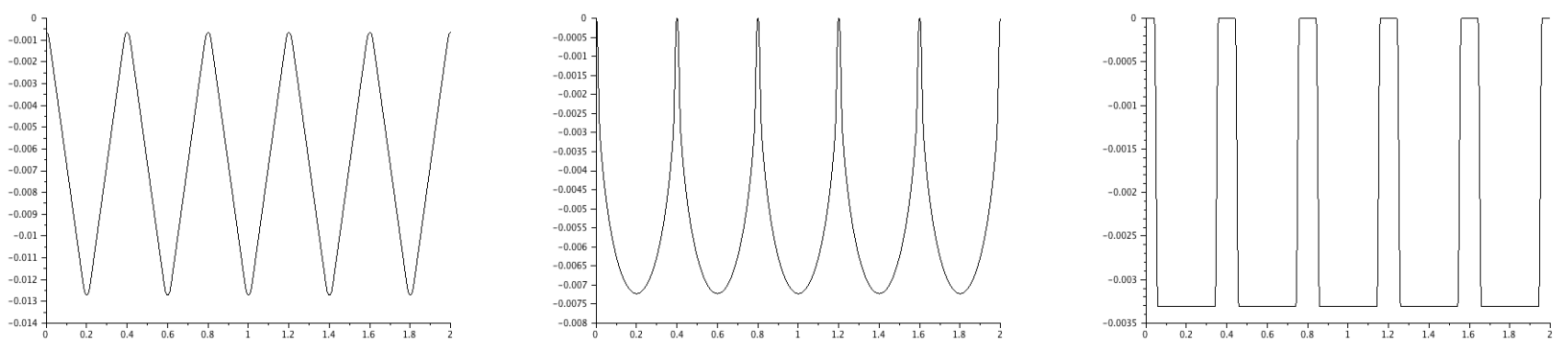

Figure 2: Sections of ribbed walls $\Gamma_{\varepsilon}$, associated with a function $\Psi$ modelling the $V$-shaped, $U$-shaped and blade-shaped riblet profile (from left to right).

\subsection{Numerical methods and algorithms}

Resolution of the auxiliary system (3.10). In order to compute coefficients $R_{1}\left(e_{1}\right), R_{1}\left(-e_{1}\right)$, one needs to solve system (3.10), with $\xi^{\prime} \in\left\{e_{1},-e_{1}\right\}$. We fix a truncature parameter $H>0$ and we consider the 
following approximated problem: find $\left(\widehat{\phi}_{H}^{\xi^{\prime}}, \widehat{\pi}_{H}^{\xi^{\prime}}\right) \in W^{1,3}\left(\mathcal{T}^{2} \times(0, H), \mathbb{R}^{3}\right) \times L^{3 / 2}\left(\mathcal{T}^{2} \times(0, H)\right)$ satisfying

$$
\left\{\begin{array}{l}
-\operatorname{div}_{\mathrm{y}}\left(\widehat{S}\left(D_{y} \widehat{\phi}_{H}^{\xi^{\prime}}\right)\right)+\nabla_{y} \widehat{\pi}_{H}^{\xi^{\prime}}=0 \quad \text { in } \mathcal{T}^{2} \times(0, H), \\
\operatorname{div}_{\mathrm{y}} \widehat{\phi}_{H}^{\xi^{\prime}}=0 \text { in } \mathcal{T}^{2} \times(0, H), \\
\widehat{\phi}_{H, 3}^{\xi^{\prime}}\left(y^{\prime}, 0\right)=-\nabla \Psi\left(y^{\prime}\right) \cdot \xi^{\prime} \text { on } \mathcal{T}^{2} \times\{0\} \\
\widehat{S}\left(D_{y} \widehat{\phi}_{H}^{\xi^{\prime}}\right)_{i, 3}=0, i=1,2, \text { on } \mathcal{T}^{2} \times\{0\} \\
\widehat{S}\left(D_{y} \widehat{\phi}_{H}^{\xi^{\prime}}\right)_{i, 3}=0, i=1,2,3, \text { on } \mathcal{T}^{2} \times\{H\}
\end{array}\right.
$$

Then, the approximate values $R_{1}^{a p p}\left(\xi^{\prime}\right)$ of $R_{1}\left(\xi^{\prime}\right)$, for $\xi^{\prime} \in\left\{e_{1},-e_{1}\right\}$, will be given by

$$
R_{1}^{a p p}\left(\xi^{\prime}\right)=\int_{\mathcal{T}^{2} \times(0, H)} \widehat{S}\left(D_{y} \widehat{\phi}_{H}^{\xi^{\prime}}\right): D_{y} \widehat{\phi}_{H}^{e_{1}} d y
$$

Such an approximation can be justified by standard arguments, relying on Proposition 3.8 that states the exponential decay of the gradient of $\widehat{\phi}^{\xi^{\prime}}$, along the $y_{3}$-direction.

Since problem (5.3) is nonlinear (due to the tensor $\widehat{S}$ ), we introduce a fixed point algorithm, that is defined as follows. Here, we drop the $\xi^{\prime}$ exponents and the $H$ subscripts to lighten the notation. Assume that $\widehat{\phi}_{k} \in W^{1,3}\left(\mathcal{T}^{2} \times(0, H), \mathbb{R}^{3}\right)$, and satisfies

$$
\operatorname{div}_{\mathrm{y}} \widehat{\phi}_{k}=0 \quad \text { in } \mathcal{T}^{2} \times(0, H), \quad \widehat{\phi}_{3, k}\left(y^{\prime}, 0\right)=-\nabla \Psi\left(y^{\prime}\right) \cdot \xi^{\prime} \text { on } \mathcal{T}^{2} \times\{0\} .
$$

Then, $\widehat{\phi}_{k+1}$ is determined as the only solution in $W^{1,3}\left(\mathcal{T}^{2} \times(0, H), \mathbb{R}^{3}\right)$, satisfying the same incompressibility and boundary condition (5.4), to the following variational formulation:

$$
\int_{\mathcal{T}^{2} \times(0, H)}\left(C_{s}\left|D \widehat{\phi}_{k}\right| \widehat{D} \phi_{k+1}-\frac{\delta^{2}}{2 \gamma} D \widehat{\phi}_{k}\left(D \widehat{\phi}_{k+1}\right)^{T}\right): D \zeta d y=0,
$$

for any test function $\zeta \in W^{1,3}\left(\mathcal{T}^{2} \times(0, H), \mathbb{R}^{3}\right)$ satisfying

$$
\operatorname{div}_{\mathrm{y}} \zeta=0 \quad \text { in } \mathcal{T}^{2} \times(0, H), \quad \zeta_{3}\left(y^{\prime}, 0\right)=0 \text { on } \mathcal{T}^{2} \times\{0\}
$$

We stop the iterations once the stopping criterion

$$
\frac{\left\|D \widehat{\varphi}_{k+1}-D \widehat{\varphi}_{k}\right\|_{L^{3}\left(\mathcal{T}^{2} \times(0, H), \mathbb{R}^{3}\right)}}{\left\|D \widehat{\varphi}_{k}\right\|_{L^{3}\left(\mathcal{T}^{2} \times(0, H), \mathbb{R}^{3}\right)}}<\epsilon
$$

is fulfilled, where $\epsilon$ is a small number (set at $\epsilon=10^{-2}$ in the numerical simulations); then, we use the approximation $\widehat{\phi}^{\xi^{\prime}} \approx \widehat{\phi}_{k+1}$.

Resolution of the effective problem (5.2). Denoting by $V$ the functional space

$$
V=\left\{w \in W^{1,3}\left(\Omega, \mathbb{R}^{3}\right), \operatorname{div} w=0 \text { in } \Omega, w_{3}=0 \text { on } \Gamma, w=0 \text { on } \Gamma^{0}\right\},
$$

endowed with the norm

$$
\|w\|_{V}:=\|D w\|_{L^{3}\left(\Omega, \mathbb{R}^{3 \times 3}\right)}^{1 / 3}
$$

the variational formulation of problem (5.2) reads: for every $\psi \in V$,

$$
\int_{\Omega}((w \cdot \nabla) w \cdot \psi+S(D w): D \psi) d x+\int_{\Gamma}\left(\beta w \cdot \psi+\lambda^{3} w_{1}^{2} R_{1}\left(\operatorname{sgn}\left(w_{1}\right) e_{1}\right) \psi_{1}\right) d \mathcal{H}^{2}=\int_{\Omega} \bar{f} \cdot \psi d x
$$


To deal with the nonlinear terms appearing in the previous formulation, we proceed in an analogous way as in the treatment of system (5.3), by introducing a fixed point algorithm. Starting from an initial guess $w^{0} \in V$, we construct a sequence of functions $w_{k} \in V$ defined recursively, as follows. For $k \in \mathbb{N}, w_{k}$ being known, we define $w_{k+1} \in V$ as the solution to the following variational formulation:

$$
\begin{aligned}
& \int_{\Omega}\left(\left(w_{k} \cdot \nabla\right) w_{k+1} \cdot \psi+S\left(D w_{k+1}\right): D \psi\right) d x+\int_{\Gamma}\left(\beta w_{k+1} \cdot \psi+\lambda^{3} w_{k, 1} w_{k+1,1} R_{1}\left(\operatorname{sgn}\left(w_{k, 1}\right) e_{1}\right) \psi_{1}\right) d \mathcal{H}^{2} \\
& \quad=\int_{\Omega} \bar{f} \cdot \psi d x, \quad \forall \psi \in V
\end{aligned}
$$

Numerically, we stop the iterations if the relative error $\left\|w_{k+1}-w_{k}\right\|_{V} /\left\|w_{k}\right\|_{V}$ is smaller than the threshold $\epsilon$ defined in the previous paragraph.

Spatial discretization by finite element methods. In order to compute the solutions to the variational formulations (5.5) and (5.7), we rely on mixed formulations of the problems, that are discretized by finite element methods using FreeFem ++ software [27]. For both systems of equations, we consider the TaylorHood approximation for the velocity-pressure pair, i.e., $P_{2}$ elements for the velocity field and $P_{1}$ elements for the pressure. It is well known that this choice is compatible with the Babuška-Brezzi condition [12,26]. The three-dimensional meshes of the cell $\mathcal{T}^{2} \times(0, H)$ and the fluid domain $\Omega$ are obtained by constrained Delaunay tetrahedralizations.

\subsection{Numerical results}

Definition of the parameters associated with the turbulence model. In order to illustrate the effect of coefficient $\lambda$, which acts directly on the component of the fluid velocity that is transverse to the riblet direction, we consider a flow driven by an external force $\bar{f}=e_{1}$. We set the Reynolds number to $R e=10000$, a value for which the use of Large Eddy Simulation may be justified in practice. For instance, in Reynolds' pipe experiment, the flow is turbulent if the Reynolds number exceeds about 4000 (see [39], p. 6).

We choose $\delta$ as the grid size of the tetrahedralization of domain $\Omega$, and define $C_{s}=\delta^{2}$ and $\gamma=6$, which appears to be standard for the numerical simulation of Smagorinsky's turbulent model (see for instance [46]). In particular, such definition of $\delta$ is coherent with the modelling of the turbulence developed in Sec. 2, since $\delta$ is a small averaging radius and the grid size is the smallest length scale accessible in the mesh. To set the value of parameter $\beta$, we follow an argument developed by Berselli et al.in [10], and define

$$
\beta=\frac{L}{\operatorname{Re} \delta},
$$

where $L$ is a characteristic length of the domain (in our case, we take $L=1$ ). To obtain formula (5.8), the previous authors have adapted Maxwell's theory of gases [36], considering the micro length scale $\delta$ as the analog of the mean free path of gas particles.

Computation of the coefficients $R_{1}\left(e_{1}\right), R_{1}\left(-e_{1}\right)$ associated with the different shapes of riblets. We have collected in Table 1 the numerical values obtained for the coefficient $R_{1}\left(e_{1}\right), R_{1}\left(-e_{1}\right)$, for the different riblet geometries. In our simulations, we have defined $H=3$ - a relatively small value that is justified by the exponential decay of the derivatives of $\widehat{\phi}$ in the vertical direction. In order to be consistent with estimate (3.12) stated in Proposition 3.8, we have normalized every function $\Psi$ so that

$$
\int_{\mathcal{T}^{1}}\left|\Psi^{\prime}\left(y_{1}\right)\right|^{3} d y_{1}=1
$$

We stress that the absolute values of those coefficients depend strongly on the geometry of the riblets, through the choice of the function $\Psi$, even though the $L^{3}$-norm of $\Psi^{\prime}$ is normalized. For instance, the 
$V$-shape coefficient is about 40 times smaller as the $U$-shape coefficient, and 80 times smaller as the blade geometry one. Another interesting feature is that, even if the value $R_{1}\left(-e_{1}\right)$ is not numerically equal to the opposite of $R_{1}\left(e_{1}\right)$, the difference appears only at the fourth digit (or at the sixth digit in the $V$-shape case). Such result may be explained by our choice of parameters, for which parameter $C_{S}$ is 12 times bigger than the factor $\delta^{2} /(2 \gamma)$. If one neglects that term, one can easily verify that the solution to system (5.3) satisfies $\widehat{\phi}^{-e_{1}}=-\widehat{\phi}^{e_{1}}$, hence $R_{1}\left(-e_{1}\right)=-R_{1}\left(e_{1}\right)$. So, we hypothesize that, with our choice of parameters, the nonlinear tensor $\widehat{S}\left(\widehat{\phi}^{\xi}\right)$ is dominated by the term $C_{s}\left|\phi^{\xi}\right| \phi^{\xi}$, which explains such apparent symmetry.

\begin{tabular}{|r||c|c|c|}
\hline & $V$-shape & $U$-shape & Blade \\
\hline \hline$R_{1}\left(e_{1}\right)$ & 0.20911 & 4.8220 & 8.6947 \\
\hline$R_{1}\left(-e_{1}\right)$ & -0.20911 & -4.8294 & -8.6903 \\
\hline
\end{tabular}

Table 1: Approximate values of the coefficients $R_{1}\left(e_{1}\right), R_{1}\left(-e_{1}\right)$, for the $U$-shape, $V$-shape and blade riblets.

Numerical results and comments. In order to establish some qualitative comparisons between the solutions to the effective system (5.2), let us introduce certain quantities that are classically associated with the stationary flow of a viscous fluid, in the vicinity of a solid wall where friction occurs:

- the kinetic energy $\frac{1}{2} \int_{\Omega}|w|^{2} d x$;

- the dissipated energy

$$
\frac{1}{2}\left[\int_{\Omega}\left(\frac{1}{R e}|D w|^{2}+C_{s}|D w|^{3}-\frac{\delta^{2}}{2 \gamma} D w(D w)^{T}: D w\right) d x+\int_{\Gamma}\left(\beta|w|^{2}+\lambda^{3} w_{1}^{3} R_{1}\left(\operatorname{sgn}\left(w_{1}\right) e_{1}\right)\right) d \mathcal{H}^{2}\right],
$$

that we decompose into two contributions:

a) the viscous dissipation

$$
\frac{1}{2} \int_{\Omega}\left(\frac{1}{R e}|D w|^{2}+C_{s}|D w|^{3}-\frac{\delta^{2}}{2 \gamma} D w(D w)^{T}: D w\right) d x
$$

b) the dissipation by friction

$$
\frac{1}{2} \int_{\Gamma}\left(\beta|w|^{2}+\lambda^{3} w_{1}^{3} R_{1}\left(\operatorname{sgn}\left(w_{1}\right) e_{1}\right)\right) d \mathcal{H}^{2}
$$

- the work of the body force $\bar{f}$, which in our case is simply defined by

$$
\int_{\Omega} w_{1} d x
$$

and will be refered to as the average streamwise velocity.

We have plotted the kinetic and dissipated energies, the viscous and frictional dissipation, and the average streamwise velocity, with respect to the value of $\lambda$, and considering $V$-shape, $U$-shape and blade riblets, in Figures 3 to 7. 


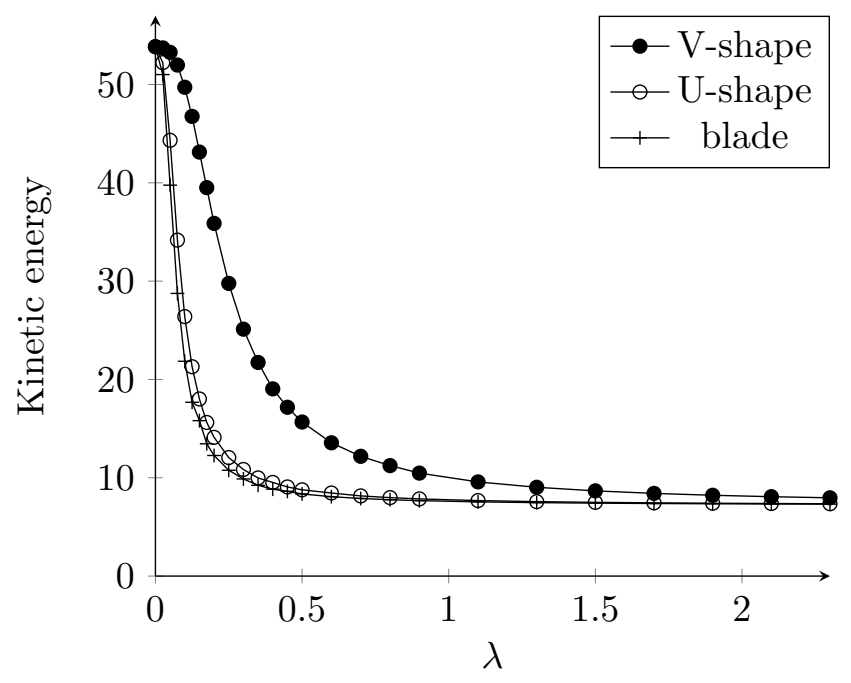

Figure 3: Kinetic energy with respect to $\lambda$, for the different riblet geometries.

We observe that for every riblet geometry, increasing $\lambda$ leads to decreasing the kinetic energy of the fluid, as well as the dissipated energy and the streamwise velocity. For $\lambda=0$, the geometry of the asperity has no effect in the model, and everything happens as if the lower wall was flat, and produced a friction measured by the coefficient $\beta$. In that case, the kinetic energy is above 50 . Then, as $\lambda$ increases, the kinetic energy diminishes drastically, before stabilizing around the value 10, which is fifth time smaller as its initial value. The profiles of the dissipated energy, its viscous part, and the streamwise velocity, are similar, but their values are only divided by a factor 2 between $\lambda=0$ and $\lambda=2.3$.

We notice that for every mechanical quantity that we have computed, the numerical values obtained for the $U$-shape and the blade riblets are very close, whereas the $V$-shape riblet behaves differently. In particular, the decreasing of the former quantities that appears for small values of $\lambda$, is much slower for the $V$-shape riblets than for the other ones. That can be explained by the proximity of the $R_{1}$ coefficients associated with the $U$-shape and the blade riblets: they only differ by a factor 2 (see Table 1 ), whereas the $V$-shape riblet produces $R_{1}$ coefficients 40 or 80 smaller in magnitude. Consequently, the effect of an increasing of $\lambda$ is less pronounced for the $V$-shape riblet, especially since the corresponding term in the variational formulation is weighted by $\lambda^{3}$, which is increasing very slowly with $\lambda$.

Among all the mechanical quantities introduced above, the frictional dissipation is the only one that presents a different behaviour. Considering the case of the $V$-shape riblet, we observe on Fig. 6 that the frictional dissipation start from about 0.3 , and then increases to reach its maximal value (around 1) for $\lambda \approx 0.5$; from that point, the value decreases, mimicing the behaviour that the kinetic or dissipated energies presents from $\lambda=0$. The origin of that initial increase of the frictional dissipation is difficult to analyze; however, Fig. 6 is an indicator of the complexity of the interaction between the fluid and the ribbed wall, which is described by a monotone term (see Remark 4.6), depending locally on the sign of the first component of the velocity. Still, it is worth noticing that in the present simulations, the viscous dissipation dominates the frictional one, so that the dissipated energy behaves similarly to the viscous one with respect to $\lambda$.

Concluding remarks. In the case of straight riblets, where the boundary conditions associated with the effective system (3.7) are given by (3.11), we have proposed a methodology that allows to compute 


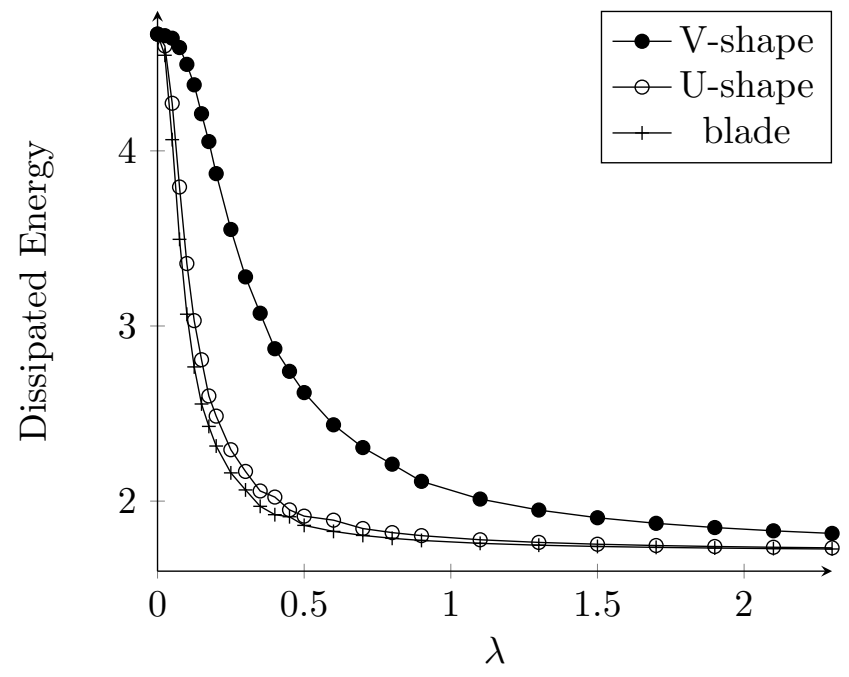

Figure 4: Dissipated Energy with respect to $\lambda$, for the different riblet geometries.

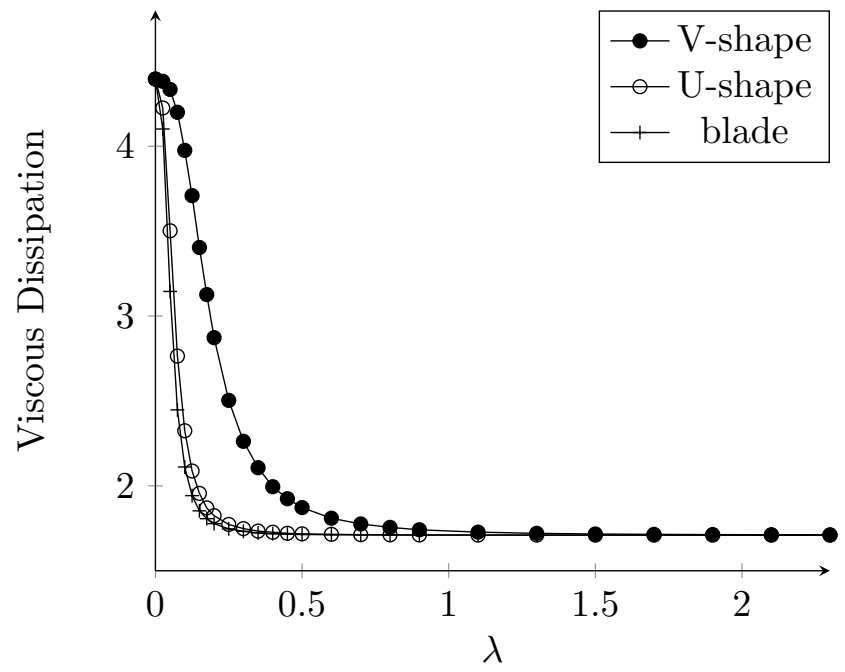

Figure 5: Viscous dissipation with respect to $\lambda$, for the different riblet geometries. 


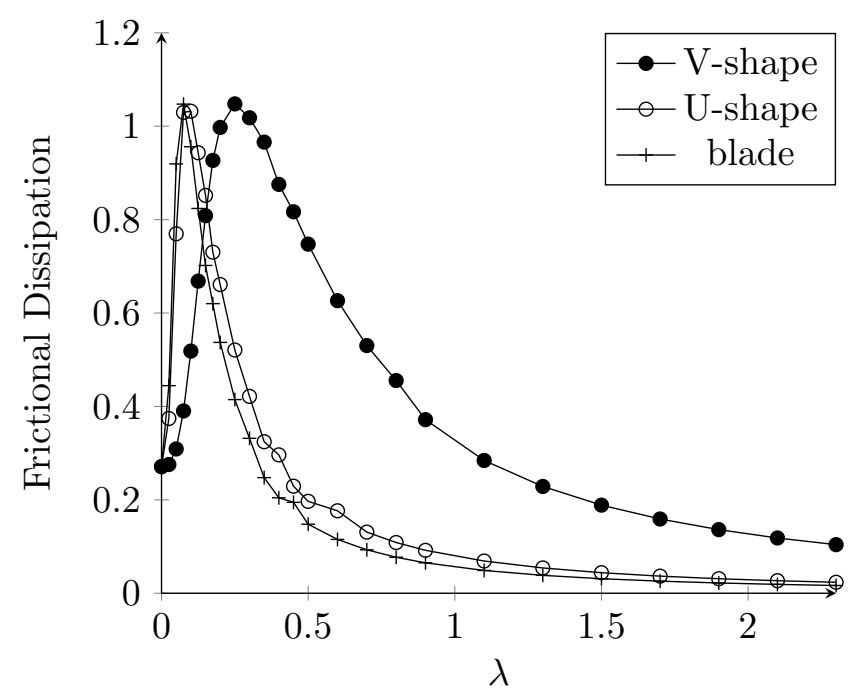

Figure 6: Frictional dissipation with respect to $\lambda$, for the different riblet geometries.

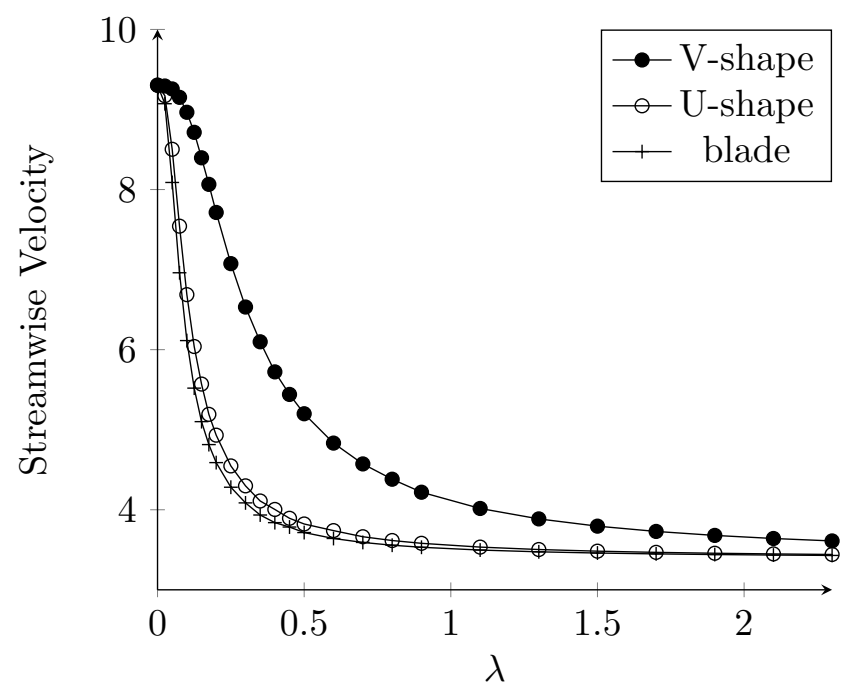

Figure 7: Average streamwise velocity $w_{1}$, with respect to $\lambda$, for the different riblet geometries. 
the required coefficients $R_{1}\left(e_{1}\right), R_{1}\left(-e_{1}\right)$, and to solve the variational formulation (5.6) by a fixed point method. Such procedure could be easily extended to treat the case of a time-dependant body force $\bar{f}$, adapting standard time discretization schemes for Navier-Stokes equations. Hence, the new boundary conditions that the asymptotic analysis detailed in Sec. 4 has pointed, are usable in practice, at least in the case of a ribbed geometry.

Besides, the analysis of the characteristics of the flow with respect to the value of coefficient $\lambda$, for different types of riblets, reveals that the effective system can be strongly affected by the extra friction term associated with $R_{1}$ and $\lambda$ in boundary conditions (3.11). By construction, that term is a non physical one, that is, a term of interaction between the fluid and the ribbed wall resulting from the averaging procedure leading to the Large Eddy Simulation model, and not from the mechanical interaction ifself. As a matter of fact, that new friction term has the same mathematical properties as the tensor $\widehat{S}$ that results from the spatial averaging of the Navier-Stokes equations; that is clear from the computations following Remark 4.6, and could explain the large influence of that extra friction term on the macroscopic features of the simulations. Hence, such effective boundary conditions as (3.11) need certainly to be taken into account in the simulation of homogenized LES models, in the vicinity of a ribbed wall.

\section{Acknowledgements}

F.J. Suárez-Grau has been supported by Ministerio de Economía y Competitividad (Spain), Proyecto Excelencia MTM2014-53309-P.

\section{References}

[1] N. A. Adams and S. Stolz, A Subgrid-Scale Deconvolution Approach for Shock Capturing, J. Comput. Phys. 178 (2002), no. 2, 391-426.

[2] G. Allaire, Homogenization and Two-Scale Convergence, SIAM Journal on Mathematical Analysis 23 (1992), no. 6, 1482-1518.

[3] Y. Amirat and J. Simon, Riblets and Drag Minimization, Contempory Mathematics 209 (1997), $9-17$.

[4] T. Arbogast, J. Douglas Jr., and U. Hornung, Derivation of the Double Porosity Model of Single Phase Flow via Homogenization Theory, SIAM Journal on Mathematical Analysis 21 (1990), no. 4, 823-836.

[5] T. G. Bagwell, Stochastic Estimation of Near Wall Closure in Turbulence Models., Ph.D. thesis, University of Illinois at Urbana-Champaign, 1994.

[6] G. I. Barenblatt and A. J. Chorin, New Perspectives in Turbulence: Scaling Laws, Asymptotics, and Intermittency, SIAM Review 40 (1998), 265-291.

[7] D. Bechert and M. Bartenwerfer, The Viscous Flow on Surfaces with Longitudinal Ribs, J. Fluid Mech. 206 (1989), no. 1, 105-129.

[8] D. W. Bechert, G. Hoppe, J. G. Th. van der Hoeven, and R. Makris, The Berlin Oil Channel for Drag Reduction Research, Experiments in Fluids 12 (1992), no. 4, 251 - 260.

[9] L. C. Berselli, G. P. Galdi, T. Iliescu, and W. J. Layton, Mathematical Analysis for the Rational Large Eddy Simulation Model, Mathematical Models and Methods in Applied Sciences 12 (2002), no. $08,1131-1152$. 
[10] L. C. Berselli, T. Iliescu, and W. J. Layton, Mathematics of Large Eddy Simulation of Turbulent Flows, Springer-Verlag Berlin Heidelberg, 2006.

[11] H. Brézis, Analyse fonctionnelle : théorie et applications, Collection Mathématiques appliquées pour la maîtrise, Masson, Paris, New York, Barcelone, 1983.

[12] F. Brezzi and M. Fortin, Mixed and Hybrid Finite Element Methods, Springer-Verlag New York, Inc., New York, NY, USA, 1991.

[13] Dorin Bucur, Eduard Feireisl, and Šárka Nečasová, On the Asymptotic Limit of Flows Past a Ribbed Boundary, Journal of Mathematical Fluid Mechanics 10 (2008), no. 4, 554-568.

[14] _ Boundary Behavior of Viscous Fluids: Influence of Wall Roughness and Friction-driven Boundary Conditions, Archive for Rational Mechanics and Analysis 197 (2010), no. 1, 117-138.

[15] J. Casado-Díaz, Exponential Decay for the Solutions of Nonlinear Elliptic Systems Posed in Unbounded Cylinders, Journal of Mathematical Analysis and Applications 328 (2007), no. 1, 151 169.

[16] J. Casado-Díaz, E. Fernández-Cara, and J. Simon, Why Viscous Fluids Adhere to Rugose Walls: a Mathematical Explanation, Journal of Differential Equations 189 (2003), no. 2, 526 - 537.

[17] J. Casado-Díaz, M. Luna-Laynez, and F. J. Suárez-Grau, Asymptotic Behavior of a Viscous Fluid with Slip Boundary Conditions on a Slightly Rough Wall, Mathematical Models and Methods in Applied Sciences 20 (2010), no. 01, 121-156.

[18] D. Cioranescu, A. Damlamian, and G. Griso, Periodic Unfolding and Homogenization, C. R. Acad. Sci. Paris 335 (2002), no. 1, $99-104$.

[19] P. Coletti, Analytical and Numerical Analysis of k-e and Large Eddy Simulation Turbulence Models, Ph.D. thesis, Dipartimento di Matematica, Università di Trento, 1998.

[20] B. Dean and B. Bhushan, Shark-Skin Surfaces for Fluid-Drag Reduction in Turbulent Flow: a Review, Philosophical Transactions of the Royal Society of London A: Mathematical, Physical and Engineering Sciences 368 (2010), no. 1933, 5737-5737.

[21] J. W. Deardorff, A Numerical Study of Three-Dimensional Turbulent Channel Flow at Large Reynolds Numbers, Journal of Fluid Mechanics 41 (1970), 453-480.

[22] C.D. Dritselis, Large Eddy Simulation of Turbulent Channel Flow with Transverse Roughness Elements on One Wall, International Journal of Heat and Fluid Flow 50 (2014), 225 - 239.

[23] G. P. Galdi, An Introduction to the Mathematical Theory of the Navier-Stokes Equations : SteadyState Problems, Springer Monographs in Mathematics, Springer, New York, NY, 2011.

[24] G. P. Galdi and W. J. Layton, Approximation of the Larger Eddies in Fluid Motions II: a Model for Space-Filtered Flow, Mathematical Models and Methods in Applied Sciences 10 (2000), no. 03, $343-350$.

[25] R. García-Mayoral and J. Jiménez, Drag Reduction by Riblets, Phil. Trans. R. Soc. A 369 (2011), $1412-1427$. 
[26] Girault, V. and Raviart, P.-A., Finite element methods for navier-stokes equations: Theory and algorithms, 1st ed., Springer Publishing Company, Incorporated, 2011.

[27] F. Hecht, New Development in FreeFem++, J. Numer. Math. 20 (2012), 251-265.

[28] W. Jäger and A. Mikelić, On the Roughness-Induced Effective Boundary Conditions for an Incompressible Viscous Flow, Journal of Differential Equations 170 (2001), no. 1, 96 - 122.

[29] _ Couette Flows over a Rough Boundary and Drag Reduction, Communications in Mathematical Physics 232 (2003), no. 3, 429-455.

[30] J. Jiménez and R. García-Mayoral, Drag Reduction by Riblets, Phil. Trans. R. Soc. A 369 (2011), $1412-1427$.

[31] O. A. Ladyzhenskaya and A. A. Kiselev, On the Existence and Uniqueness of the Solution of the Nonstationary Problem for a Viscous, Incompressible Fluid, Izv. Akad. Nauk SSSR Ser. Mat. 21 (1957), 655-680.

[32] E. Lauga, M. Brenner, and H. Stone, Microfluidics: The No-Slip Boundary Condition, pp. 12191240, Springer Berlin Heidelberg, Berlin, Heidelberg, 2007.

[33] S.-J. Lee and S.-H. Lee, Flow Field Analysis of a Turbulent Boundary Layer over a Riblet Surface, Experiments in Fluids 30 (2001), no. 2, 153-166.

[34] J. Leray and J.-L. Lions, Quelques résultats de Višik sur les problèmes elliptiques non linéaires par les méthodes de Minty-Browder, Bulletin de la Société Mathématique de France 93 (1965), 97-107.

[35] J.-L. Lions, Quelques méthodes de résolution des problèmes aux limites non linéaires, Dunod Paris, 1969.

[36] J. C. Maxwell, On Stresses in Rarified Gases Arising from Inequalities of Temperature, Phil. Trans. R. Soc. Lond. 170 (1879), 231-256.

[37] J. M. McDonough, Introductory Lectures on Turbulence. Physics, Mathematics and Modeling, 2004.

[38] G. Nguetseng, A General Convergence Result for a Functional Related to the Theory of Homogenization, SIAM Journal on Mathematical Analysis 20 (1989), no. 3, 608-623.

[39] S. B. Pope, Turbulent Flows, Cambridge University Press, 2000.

[40] P. Sagaut, Large Eddy Simulation for Incompressible Flows, Springer-Verlag Berlin Heidelberg, 2006.

[41] J. Serrin, Mathematical Principles of Classical Fluid Mechanics, Springer Berlin Heidelberg, 1959.

[42] J. S. Smagorinsky, General Circulation Experiments with the Primitive Equations, Mon. Weather Review 164 (1963), 91-99.

[43] F.J. Suárez-Grau, Effective Boundary Condition for a Quasi-Newtonian Viscous Fluid at a Slightly Rough Boundary Starting from a Navier Condition, ZAMM - Journal of Applied Mathematics and Mechanics / Zeitschrift für Angewandte Mathematik und Mechanik 95 (2015), no. 5, 527-548.

[44] L. Tartar, An Introduction to Navier-Stokes Equation and Oceanography, Lecture Notes of the Unione Matematica Italiana, Springer-Verlag Berlin Heidelberg, 2006. 
[45] P.R. Viswanath, Aircraft Viscous Drag Reduction using Riblets, Progress in Aerospace Sciences 38 (2002), 571-600.

[46] J. Volker, Large Eddy Simulation of Turbulent Incompressible Flows, Lecture Notes in Computational Science and Engineering, vol. 34, Springer-Verlag Berlin Heidelberg New York, 2004. 